\title{
Estabilidade estrutural das equações diferenciais de segunda ordem polinomiais com coeficientes periódicos
}

Adolfo Washington Guzmán

\author{
TESE APRESENTADA \\ $\mathrm{AO}$ \\ INSTITUTO DE MATEMÁTICA E ESTATÍSTICA \\ DA \\ UNIVERSIDADE DE SÃO PAULO \\ PARA \\ OBTENÇÃO DO GRAU DE DOUTOR \\ EM \\ MATEMÁTICA APLICADA \\ Área de Concentração: Matemática Aplicada \\ Orientador: Prof. Dr. Jorge Manuel Sotomayor Tello
}

Durante a elaboração deste trabalho o autor recebeu apoio financeiro do CNPq.

São Paulo, fevereiro de 2003 


\title{
Estabilidade estrutural das equações diferenciais de segunda ordem polinomiais com coeficientes periódicos
}

\author{
Este exemplar corresponde à redação \\ final da tese devidamente corregida \\ e defendida por Adolfo Washington Guzmán \\ e aprovada pela comissão julgadora.
}

São Paulo, fevereiro de 2003.

Banca examinadora:

- Prof. Dr. Jorge Manuel Sotomayor Tello (orientador) (IME-USP)

- Prof. Dr. Clodoaldo Grotta Ragazzo (IME-USP)

- Prof. Dr Ronaldo Alves Garcia (UFG)

- Prof. Dr. Marco Antonio Teixeira (IMECC-UNICAMP)

- Prof. Dr. Luiz Fernando Osorio Mello (UNIFEI) 
a meu pai, a minha mãe, in memoria a Sônia. 


\section{Agradecimentos}

Quero expressar meu agradecimento ao Prof. Jorge Sotomayor por ter proposto o problema que deu lugar ao presente trabalho. Sou grato por sua confiança e, sobretudo, por ter compartilhado seu conhecimento.

Agradeço, também,

aos membros da banca examinadora, em particular aos Professores Ronaldo Alves Garcia e Luiz Fernando Osorio Mello pelas correções por eles apontadas e sugestões sobre futuras questões.

aos professores e alunos da Pós-graduação do IME que ajudaram a manter um clima agradável e cordial durante estes quase quatro anos,

aos amigos e companheiros Miguel Abadi, Jesus Garcia e Viviana Giampaoli,

e a minha melhor amiga Sônia Fernandes. 


\section{Resumo}

No cilindro $M=S^{1} \times \Re$ como espaço de fase caracterizamos os campos vetoriais estruturalmente estáveis que provêm das equações diferenciais de segunda ordem $E_{f}: x^{\prime \prime}=f\left(x, x^{\prime}\right)$ onde $f(x, y)=\sum_{i=0}^{n} a_{i}(x) y^{i}$ e $a_{i}(x)$ são funções periódicas de classe $C^{r} \operatorname{com} r \geq 1$ e $n \geq 1$.

Denotamos por $\mathcal{E}^{n, r}$ o espaço dos campos vetoriais $X(f)=y \frac{\partial}{\partial x}+f(x, y) \frac{\partial}{\partial y}$ associados às equações $E_{f} \operatorname{com} f(x, y)=\sum_{i=0}^{n} a_{i}(x) y^{i}$.

Através de apropriadas compactificações do campo $X(f)$ e do espaço de fase $M$, que denotamos $\widetilde{X}(f)$ e $\widetilde{M}$ respectivamente, descrevemos, para cada $n$, o comportamento das trajetórias em uma vizinhança do infinito. Os pontos infinitos de $M$ são representados pela fronteira de $\widetilde{M}$. Assim, para $n=1,2$, encontramos na fronteira órbitas periódicas; para $n=3$, pontos de tangências; para $n=4$, singularidades hiperbólicas; e para $n>4$, singularidades do tipo semi-hiperbólico ou nilpotente.

A caracterização dos campos vetoriais $X(f)$ estruturalmente estáveis sobre $M$ é estabelecida em termos das propriedades dos campos $\widetilde{X}(f)$ definidos em $\widetilde{M}$ que não se alteram por pequenas perturbações de classe $C^{r}$ das funções coeficientes $a_{i}$.

Para cada $n \geq 1$, determinamos um conjunto $\Sigma^{n}$ contido em $\mathcal{E}^{n, r}$ com propriedades simples com respeito as singularidades, as órbitas periódicas e as conexões de separatrizes de singularidades de $\widetilde{X}(f)$. Provamos que $\Sigma^{n}$ é aberto e denso em $\mathcal{E}^{n, r}$ e que caracteriza os campos estruturalmente estáveis. 


\section{Abstract}

In the cylinder $M=S^{1} \times \Re$ as phase space, we characterize the structurally stable vector fields that derive from the second order differential equations $E_{f}: x^{\prime \prime}=f\left(x, x^{\prime}\right)$ where $f(x, y)=\sum_{i=0}^{n} a_{i}(x) y^{i}$ for $a_{i}(x) C^{r}$-periodic functions with $r \geq 1$ and $n \geq 1$.

We denote by $\mathcal{E}^{n, r}$ the space of vector fields $X(f)=y \frac{\partial}{\partial x}+f(x, y) \frac{\partial}{\partial y}$ associated to $E_{f}$ with $f(x, y)=\sum_{i=0}^{n} a_{i}(x) y^{i}$.

By appropriate compactifications of the vector field $X(f)$ (denoted $\tilde{X}(f)$ ) and the phase space $M($ denoted $\widetilde{M})$, we describe the trajectory behavior in a neighborhood of infinity. The infinite points of $M$ are represented in the boundary of $\widetilde{M}$. Thus, we find on the boundary periodic orbits for $n=1,2$; points of tangency for $n=3$; hyperbolic singularities for $n=4$; and semihyperbolic or nilpotent singularities for $n>4$.

We establish the structural stability of the vector fields $X(f)$ on $M$ using the properties of $\widetilde{X}(f)$ in $\widetilde{M}$ which do not modify for small $C^{r}$-perturbations of the functions $a_{i}$.

For each $n \geq 1$, we determine a set $\Sigma^{n}$ in $\mathcal{E}^{n, r}$ which has simple properties with respect to singularities, periodic orbits and singularity separatrix connection of $\widetilde{X}(f)$. Also, we prove that $\Sigma^{n}$ is open and dense in $\mathcal{E}^{n, r}$ and that it characterizes the structurally stable vector fields. 


\section{Sumário}

Introdução $\quad$ v

1 Preliminares 1

1.1 Campos vetoriais e equações de segunda ordem . . . . . . . . 1

1.2 Comportamento qualitativo . . . . . . . . . . . . 2

1.2 .1 Singularidades . . . . . . . . . . . . . . 3

1.2 .2 Órbitas periódicas . . . . . . . . . . 5

1.3 Compactificação . . . . . . . . . . . . . . . 6

1.3.1 Compactificação cilíndrica . . . . . . . . . . 6

1.3.2 Transformação das vizinhanças do infinito de $M$. . . 7

1.4 Comportamento qualitativo no infinito . . . . . . . . . 8

1.5 Estabilidade Estrutural . . . . . . . . . . . . . . . . . 10

2 Campos de vetores $X(f)$ associados as equações de segunda ordem 13

2.1 Comportamento das trajetórias de $X(f) \ldots \ldots . . . . . .13$

2.1.1 Singularidades. . . . . . . . . . . . . 14

2.2 Compactificação de $X(f) \ldots \ldots \ldots \ldots$

2.2.1 Compactificação cilíndrica . . . . . . . . . . 16

2.2.2 Uma representação local de $X(f)$ no infinito . . . . . 17

2.3 Comportamento das órbitas de $\widetilde{X}(f) \ldots \ldots \ldots . . \ldots . . \ldots 17$

3 Estabilidade estrutural de $X(f) \quad 32$

3.1 Caso $n=1 \ldots \ldots \ldots \ldots 33$

3.1 .1 Definição de $\Sigma^{1} \ldots \ldots \ldots \ldots$. . . . . . . . . . . 33

3.1 .2 Genericidade de $\Sigma^{1} \ldots \ldots \ldots \ldots . \ldots \ldots$

3.2 Caso $n=2 \ldots \ldots \ldots \ldots$. . . . . . . . . . . . . . . 40

3.2 .1 Definição de $\Sigma^{2} \ldots \ldots \ldots \ldots$. . . . . . . . 40

3.2 .2 Genericidade de $\Sigma^{2} \ldots \ldots \ldots \ldots$. . . . . . 41

3.3 Caso $n=3 \ldots \ldots \ldots \ldots \ldots$. . . . . . . . . . . . . . . . . . . . . . . . . . . . .

3.3 .1 Definição de $\Sigma^{3} \ldots \ldots \ldots \ldots$. . . . . . . . 44 
3.3.2 Genericidade de $\Sigma^{3} \ldots \ldots \ldots \ldots . \ldots . \ldots 44$

3.4 Caso $n=4 \ldots \ldots \ldots \ldots$

3.4 .1 Definição de $\Sigma^{4} \ldots \ldots \ldots \ldots \ldots$

3.4 .2 Genericidade de $\Sigma^{4} \ldots \ldots \ldots \ldots \ldots$

3.5 Caso $n \geq 5$. . . . . . . . . . . . . . . 56

3.5.1 Definição de $\Sigma^{n} \ldots \ldots \ldots \ldots . \ldots . \ldots 57$

3.5.2 Genericidade de $\Sigma^{n} \ldots \ldots \ldots \ldots 57$

3.6 Caracterização da Estabilidade Estrutural . . . . . . . . . . . 60

3.6.1 Necessidade . . . . . . . . . . . . . . . . . 61

3.6.2 Suficiência . . . . . . . . . . . . 65

3.7 Exemplos de $X(f)$ estruturalmente estável . . . . . . . . . 73 


\section{Introdução}

Um campo vetorial $X$ é estruturalmente estável se os campos $Y$ suficientemente próximos mantêm o comportamento de suas trajetórias topologicamente equivalente ao de $X$.

O objetivo deste trabalho é caracterizar os campos vetoriais estruturalmente estáveis dentro da classe dos campos vetoriais associados a um tipo de equações diferenciais de segunda ordem definidas no cilindro $M=S^{1} \times \Re$.

Denotamos por $E_{f}$ a equação diferencial de segunda ordem $x^{\prime \prime}=f\left(x, x^{\prime}\right)$ e por $X(f)$ o campo vetorial associado $X(f)=y \frac{\partial}{\partial x}+f(x, y) \frac{\partial}{\partial y}$ onde $f \in$ $C^{r}(M)$.

Neste trabalho, consideramos os campos $X(f)$ cuja função $f$ é da forma $f(x, y)=\sum_{i=0}^{n} a_{i}(x) y^{i}$ onde $a_{i}(x)$ são funções periódicas de $\Re$ em $\Re$ de classe $C^{r}$ e $n$ é um inteiro positivo.

Em [2], aparecem aplicações destas equações de segunda ordem descrevendo sistemas físicos, por exemplos, os sistemas electromecânicos, que são convenientemente modelados sobre um espaço de fase cilíndrico. $\mathrm{O}$ interesse nesses sistemas é determinar os movimentos oscilatórios ou órbitas periódicas.

Denotamos por $\mathcal{E}^{n, r}(M)$ (ou simplesmente $\mathcal{E}^{n, r}$ ) o espaço dos campos vetoriais $X(f)$ definidos em $M$, munido da topologia dada pela norma $C^{r}$ dos coeficientes com $n$ fixo e $r \geq 1$.

A ferramenta principal que usaremos para estudar a estabilidade estrutural de $X(f)$ sobre $M=S^{1} \times \Re$ é a compactificação de $M$ e $X(f)$ que denotaremos por $\widetilde{M}$ e $\widetilde{X}(f)$ respectivamente. Ver a definição de $\widetilde{M}$ e $\widetilde{X}$ na seção 1.3 .

Para cada $n \geq 1$, estabelecemos o conjunto $\Sigma^{n}(M)$ de campos $X(f)$ que satisfazem as seguintes propriedades tendo em conta o comportamento dos campos associados $\widetilde{X}(f)$

1. Todas suas singularidades contidas em $C_{0}=S^{1} \times\{0\}$ são hiperbólicas $e$ as singularidades no infinito são hiperbólicas ou semi-hiperbólicas dependendo de $n$.

2. Todas suas órbitas periódicas contidas no interior de $\widetilde{M}$ são hiperbólicas. 
Só para $n=1,2$ existem órbitas periódicas no infinito.

3. Para $n=3$, as órbitas que não são separatrizes de singularidade podem tangenciar a fronteira de $\widetilde{M}$ no máximo uma vez.

4. Não têm conexões de separatrizes de singularidades tanto sejam singularidades finitas quanto localizadas no infinito. Em particular, não têm conexões de selas.

Os resultados principais deste trabalho são:

Genericidade: $\Sigma^{n}(M)$ é aberto e denso em $\mathcal{E}^{n}$.

Caracterização: $X(f) \in \mathcal{E}^{n, r}$ é estruturalmente estável se e somente se $X(f) \in \Sigma^{n}(M)$.

Damos as definições de $\Sigma^{n}$ e os enunciados de maneira mais precisa no Capítulo 3.

Este trabalho está inserido no estudo da estabilidade estrutural dos campos vetoriais definidos em variedades abertas.

A caracterização dos campos vetoriais estruturalmente estáveis definidos sobre variedades abertas é abordado através de dois enfoques.

Primeiramente, estudam-se os fluxos dos campos no plano definindo propriedades das trajetórias com ênfase nas que escapam ao infinito. Em particular pede-se que cada trajetória tendendo ao infinito tenha uma vizinhança tubular de trajetórias seguindo um mesmo comportamento. Em outro caso, acrescenta-se ao espaço de fase um conjunto enumerável de pontos chamados de pontos "finais" ou "infinitos". Estes pontos permitem definir um comportamento das trajetórias no infinito chamado de "sela no infinito". Discutiremos este caso na Seção 1.4.

No segundo enfoque, que será o que desenvolveremos aqui, associa-se um campo vetorial definido sobre uma variedade compacta de tal maneira que o infinito da variedade aberta fica agora bem determinado pela fronteira da compacta. O comportamento das trajetórias em uma vizinhança do infinito pode ser agora estudado sobre regiões finitas.

A seguir, detalhamos as conclusões mais relevantes de ambos enfoques.

Sob o primeiro enfoque, vários trabalhos foram dedicados a caracterização de campos vetoriais estruturalmente estáveis. Em [15], apresenta-se a caracterização dos fluxos sobre uma superfície aberta $N$. Ali, determinou-se para um fluxo $\varphi$ em $N$, que as condições

K.1 Todos os pontos periódicos (incluídos os fixos) são hiperbólicos. 
K.2 Não tem conjuntos minimais não triviais nem órbitas oscilantes.

K.3 Não tem conexões de selas tanto sejam fixas quanto definidas no infinito.

são suficientes para a estabilidade estrutural global $C^{r} \operatorname{com} r \geq 1$. Além disso, provou-se que essas condições são também necessárias quando $N=\Re^{2}$. Em [6], mostrou-se que essas condições podem ser consideradas necessárias para a estabilidade em qualquer superfície aberta de gênero finito e $r=1$. Para $r \geq 1$, em [14] provou-se que essas mesmas condições para campos vetoriais são necessárias para a estabilidade estrutural global $C^{r}$ sobre superfícies abertas orientáveis com gênero finito e com um espaço enumerável de pontos finais formando a fronteira ideal de $N$.

Um primeiro estudo das equações diferenciais de segunda ordem sobre uma superfície aberta foi dado por Barreto em [4]. Aí, foram considerados $N=\Re^{2}$, as funções $f(x, y) \in C^{1}$ periódicas em $x$ e a topologia $C^{1}$-forte (ou de Whitney). Com essas condições, determinou-se o conjunto $\Sigma_{\mathcal{E}\left(\Re^{2}\right)}^{1}$ formado pelos campos com as seguintes propriedades:

B.1 Todas as singularidades são hiperbólicas.

B.2 Todas as órbitas periódicas são hiperbólicas.

B.3 Os conjuntos $\alpha$ e w-limites de qualquer trajetória só podem ser uma singularidade, ou uma órbita periódica ou infinito.

B.4 Se uma trajetória $\gamma$ tem $\alpha$-limite (resp. $\omega$-limite) uma sela, então toda trajetória contida em uma vizinhança tubular de $\gamma$ tem o mesmo $\omega$-limite (resp. $\alpha$-limite). Em particular nenhuma trajetória liga selas.

Também, provou-se que esse conjunto é aberto e denso na topologia $C^{1}$ forte e as condições dadas são necessárias e suficientes para a estabilidade estrutural.

Por outro lado, sob o segundo enfoque, a caracterização dos campos vetoriais estruturalmente estáveis é determinada pela observação das propriedades mais simples dos campos vetoriais associados por um processo de compactificação. Esse procedimento teve origem com Poincaré ao estudar os campos polinomiais definidos no plano $\Re^{2}$ projetados centralmente sobre a esfera $S^{2}$. Desta maneira o estudo das trajetórias numa vizinhança do infinito é realizado numa vizinhança do equador $S^{1}$ da esfera. Gonzales Velasco, em [12], e Sotomayor, em [19], usaram o processo de compactificação de Poincaré para caracterizar os campos polinomiais estruturalmente estáveis em $\Re^{2}$.

Em [12], determinou-se que os campos polinomiais compactificados com todas as singularidades hiperbólicas em $S^{1}$, ou com $S^{1}$ constituindo uma 
órbita periódica hiperbólica, formam um conjunto aberto e denso no espaço dos campos polinomiais estruturalmente estáveis sobre $\Re^{2}$.

As propriedades de estabilidade e genericidade dos campos polinomiais no plano foram dadas por Sotomayor em [21]. Nesse trabalho, foi levantada uma importante questão em relação a se a hiperbolicidade das órbitas periódicas contidas em $S^{2}-S^{1}$ é necessária para a estabilidade estrutural dos campos polinomiais.

Deste modo, a caracterização dos campos vetoriais estruturalmente estáveis sobre uma superfície aberta se reduz a estudar os campos sobre uma região compacta do plano.

Sobre regiões compactas conexas $N \subset \Re^{2}$ com fronteira, mostrou-se em [20] que o conjunto $\Sigma_{\mathcal{E}(N)}^{r}$ dos campos vetoriais $X(f)$ com as propriedades:

S.1 Todas suas singularidades são hiperbólicas e contidas no interior de $N$.

S.2 Todas as órbitas periódicas são hiperbólicas e contidas no interior de $N$.

S.3 $X(f)$ e $\partial N$ (a fronteira de $N$ ) são transversais ou tangentes com contato parabólico.

S.4 Não tem conexões de selas em $N$, nem separatrizes de sela tangentes a $\partial N$ ou passando por uma esquina de $N$, e nem órbitas que tangenciem a $\partial N$ em pontos diferentes.

constitui um conjunto aberto e denso em $C^{r}(N)$ com a topologia $C^{r}$-uniforme com $r \geq 1$. Além disso, mostrou-se que essas propriedades de $X(f)$ são condições necessárias e suficientes para a estabilidade estrutural.

\section{Estrutura do trabalho}

Neste trabalho, começamos estabelecendo, no capítulo 1, os conceitos preliminares referentes aos campos vetoriais sobre $\Re^{2}$ e às equações diferenciais de segunda ordem. Apresentamos a compactificação de $X(f)$ e de $M$. E por último, apresentamos os conceitos básicos da estabilidade estrutural, entre eles, a definição de estabilidade estrutural e de região canônica.

No capítulo 2, apresentamos o comportamento dos campos $X(f)$ e de sua compactificação $\widetilde{X}(f)$. Damos uma expressão do campo $\tilde{X}(f)$, determinando além do número e tipo de singularidades, as características das trajetórias perto do infinito de $M$. Por exemplo, encontramos na fronteira de $\widetilde{M}$ órbitas periódicas quando $n=1 \mathrm{e} 2$, pontos de tangência quando $n=3$, e singularidades hiperbólicas, semi-hiperbólicas e nilpotentes quando $n \geq 4$. 
No capítulo 3 , definimos para cada $n \geq 1$ o conjunto $\Sigma^{n}$ e provamos os Teoremas de Genericidade e de Caracterização enunciados anteriormente. A complexidade de cada caso dependente de $n$ reside na presença ou não de singularidades no infinito.

$\mathrm{Na}$ seção 3.6.1, provamos a necessidade das condições de $\Sigma^{n}$ para a estabilidade estrutural. Para os campos estudados aqui, que são polinomiais em uma variável, damos uma resposta afirmativa à questão levantada por Sotomayor com respeito à necessidade da hiperbolicidade das órbitas periódicas contidas em $M$ para a estabilidade estrutural.

Na seção 3.6.2, provamos a caracterização de $X(f) \in \mathcal{E}^{n, r}$ estruturalmente estável, introduzindo novas regiões canônicas em comparação às encontradas por Peixoto-Peixoto [18] e Sotomayor [19]. As novas regiões canônicas surgem pela consideração das singularidades na fronteira de $\widetilde{M}$.

Finalmente, na seção 3.7, damos alguns exemplos de campos $X(f) \in \Sigma^{n}$ que formam as diferentes regiões canônicas. 


\section{Capítulo 1}

\section{Preliminares}

Neste capítulo apresentamos os conceitos básicos das equações diferenciais de segunda ordem e dos campos vetoriais associados.

Estudamos o comportamento qualitativo dos campos vetoriais, especialmente o comportamento das trajetórias não-limitadas que escapam ao infinito. Com compactificações apropriadas, descrevemos o comportamento no infinito.

Estabelecemos os conceitos da estabilidade estrutural dos campos vetoriais definidos sobre o cilindro $M=S^{1} \times \Re$.

\subsection{Campos vetoriais e equações de segunda ordem}

Seja $M$ um subconjunto aberto de $\Re^{2}$. Um campo vetorial no plano é uma aplicação $X: M \rightarrow \Re^{2}$ de classe $C^{r}$ tal que $(x, y) \mapsto(P(x, y), Q(x, y))$. As funções $P(x, y)$ e $Q(x, y)$ são chamadas de funções componentes de $X$.

Associamos ao campo $X=(P, Q)$ o sistema de equações diferenciais

$$
\left\{\begin{array}{l}
x^{\prime}=P(x, y) \\
y^{\prime}=Q(x, y)
\end{array} .\right.
$$

Uma equação diferencial de segunda ordem é a equação

$$
x^{\prime \prime}=f\left(x, x^{\prime}\right)
$$

onde $f: M \subseteq \Re^{2} \rightarrow \Re$ é uma função de classe $C^{r}$.

A equação (1.2) é associada a um campo vetorial (que denotamos por) $X(f)$ cujas funções componentes são dadas pelas funções $P(x, y)=y \mathrm{e}$ $Q(x, y)=f(x, y)$. 
As soluções $\gamma: I \rightarrow M$ de (1.1) onde $I$ é um intervalo de $\Re$ maximal, são chamadas curvas integrais ou trajetórias. A imagem de $\gamma$ em $M$ é chamada órbita que denotamos por $\mathcal{O}(\gamma)$. Algumas vezes, indicaremos por $\mathcal{O}(\gamma, p)$ a órbita associada à trajetória que passa por $p$.

O espaço de fase do campo $X$ é o domínio $M$ decomposto pelas suas curvas integrais.

O fluxo de um campo $X$ é a aplicação $\Phi: \Re \times M \rightarrow \Re^{2}$ tal que $\Phi_{p}(t)=$ $\Phi(t, p)$ é uma curva integral do campo que passa por $p$ e tem $I=\Re$.

\subsection{Comportamento qualitativo}

Dado $p \in M$, denotamos por $\gamma(\cdot, p)$ a curva integral $\gamma: \Re \rightarrow M$ do campo $X$ que passa por $p$.

Definimos os conjuntos $\alpha$ e $\omega$-limites de $p$ como segue

$$
\begin{aligned}
& \alpha(p)=\left\{q \in M: \exists t_{n} \rightarrow-\infty \text { tal que } \gamma\left(t_{n}, p\right) \rightarrow q \text { quando } n \rightarrow+\infty\right\} \\
& \omega(p)=\left\{q \in M: \exists t_{n} \rightarrow+\infty \text { tal que } \gamma\left(t_{n}, p\right) \rightarrow q \text { quando } n \rightarrow+\infty\right\} .
\end{aligned}
$$

Uma órbita de $X$ que passa por $p$ é descrita pelo conjunto

$$
\mathcal{O}(\gamma, p)=\{\gamma(t, p): t \in \Re\}
$$

A semi-órbita positiva (resp. negativa) é o conjunto $\mathcal{O}^{+}(\gamma, p)=\{\gamma(t, p): t>$ $0\}\left(\right.$ resp. $\left.\mathcal{O}^{-}(\gamma, p)=\{\gamma(t, p): t<0\}\right)$.

Dizemos que uma semi-órbita $\mathcal{O}^{ \pm}(\gamma, p)$ é limitada se e só se existe um compacto $K$ contido em $M$ tal que $\mathcal{O}^{ \pm}(\gamma, p) \subset K$. E que uma semi-órbita $\mathcal{O}^{ \pm}(\gamma, p)$ escapa ao infinito se e só se para qualquer compacto $K$ contido em $M$, existe um ponto $q \in \mathcal{O}^{ \pm}(\gamma, p)$ tal que $\mathcal{O}^{ \pm}(\gamma, q) \cap K=\emptyset$.

Em termos dos conjuntos $\alpha$ e $\omega$-limites, dizemos que uma semi-órbita positiva (resp. negativa) é limitada se $\omega(p)$ (resp. $\alpha(p)$ ) é compacto; e que escapa ao infinito se $\omega(p)=\emptyset($ resp. $\alpha(p)=\emptyset)$.

$\mathrm{O} \omega$-limite (resp. $\alpha$-limite) de uma órbita $\mathcal{O}(\gamma, p)$ é o conjunto $\omega$-limite (resp. $\alpha$-limite) de qualquer ponto $q \in \mathcal{O}(\gamma, p)$. Mais precisamente $\omega(\mathcal{O}(\gamma, p))=$ $\omega(q)$ para qualquer $q \in \mathcal{O}(\gamma, p)$.

Um conjunto $V \subset M$ é invariante pelo fluxo do campo $X$ se $\forall p \in V$, a órbita que passa por $p, \mathcal{O}(\gamma, p)$, está contida inteiramente em $V$.

A variedade estável de $V$ é o conjunto

$$
W^{s}(V)=\{p \in M: \omega(p) \subset V\} .
$$

A variedade instável de $V$ é o conjunto

$$
W^{u}(V)=\{p \in M: \alpha(p) \subset V\} .
$$


Existem dois tipos particulares de órbitas que permitem descrever em parte o espaço de fase de um campo vetorial. A saber, as singularidades e as órbitas periódicas.

\subsubsection{Singularidades}

As órbitas $\mathcal{O}(\gamma, p)=\{p\}$ são chamadas de singularidades do campo $X=$ $(P, Q)$. São determinadas pelos zeros das funções componentes de $X$. Em outras palavras, $p$ é uma singularidade de $X$ se $P(p)=0$ e $Q(p)=0$.

Para classificar e descrever o retrato de fase de uma singularidade $p$, começamos estudando a parte linear do campo, $D X(p)=\left(\begin{array}{cc}\frac{\partial P}{\partial x}(p) & \frac{\partial P}{\partial y}(p) \\ \frac{\partial Q}{\partial x}(p) & \frac{\partial Q}{\partial y}(p)\end{array}\right)$. $\mathrm{Na}$ verdade, associamos à parte linear uma equação diferencial linear da forma

$$
\left(\begin{array}{l}
x^{\prime} \\
y^{\prime}
\end{array}\right)=D X(p)\left(\begin{array}{l}
x \\
y
\end{array}\right) \text {. }
$$

Sejam $\lambda_{1}$ e $\lambda_{2}$ os autovalores de $D X$, e $v_{1}$ e $v_{2}$ os autovetores associados a $\lambda_{1}$ e $\lambda_{2}$ respectivamente. Encontramos, então, os seguintes tipos de singularidades do campo linear segundo os valores das partes reais de $\lambda_{1} \mathrm{e}$ $\lambda_{2}$.

- Se $\lambda_{1}$ e $\lambda_{2}$ são reais e $\lambda_{1} \cdot \lambda_{2}<0$ então $p$ é dito uma sela. As variedades estável e instável são os espaços próprios associados aos autovalores negativo e positivo respectivamente. Também dizemos que a variedade estável (resp. instável) é uma órbita que tem $p$ como $\omega$-limite (resp. $\alpha$-limite).

- Se $\lambda_{1}$ e $\lambda_{2}$ são reais e $\lambda_{1}<\lambda_{2}<0$ (resp. $\lambda_{1}>\lambda_{2}>0$ ) então $p$ é dito um nó atrator (resp. repulsor). Todas as órbitas têm $p$ como $\omega$-limite (resp. $\alpha$-limite).

- Se $\lambda_{1}$ e $\lambda_{2}$ são reais e $\lambda_{1}=\lambda_{2} \neq 0$ então $p$ é dito um nó degenerado. Se $D X$ é diagonalizável, $p$ é dito um nó diacrítico, e se é nãodiagonalizável, é dito um nó confluente.

- Se $\lambda_{1}$ e $\lambda_{2}$ são complexos conjugados então $p$ é dito um foco se $\operatorname{Re}\left(\lambda_{i}\right) \neq$ 0 , ou centro se $\operatorname{Re}\left(\lambda_{i}\right)=0$.

Um ponto singular $p$ é dito hiperbólico se os valores próprios de $D X(p)$ não têm parte real nula. É dito semi-hiperbólico se somente um dos valores 
próprios é zero. E é dito nilpotente se $\operatorname{det}(D X(p))$ e traço $(D X(p))$ são nulos e $D X(p)$ não é identicamente nula.

A seguinte proposição permite descrever o retrato de fase numa vizinhança das singularidades hiperbólicas de um sistema não-linear observando sua parte linear.

Proposição 1.1 (Hartman-Grobman) Dado um campo $X$ definido em $M$ de classe $C^{1}$. Seja $p_{*}$ uma singularidade hiperbólica de $X$. Então existem um homeomorfismo h definido numa vizinhança de $p_{*}$ sobre uma vizinhança da origem com $h\left(p_{*}\right)=(0,0)$ e um intervalo $I \subset \Re$ que contém a origem tais que

$$
h \circ \gamma(t, p)=e^{t D X\left(p_{*}\right)} h(p)
$$

$\forall t \in I \quad e \forall p \in M$.

Uma prova da Proposição 1.1 pode ser encontrada em [17].

O primeiro caso de uma singularidade $p$ de $X$ que já não é hiperbólica acontece quando um dos autovalores de $D X$ é nulo. Suponhamos que $\lambda_{1}=0$ e $\lambda_{2} \neq 0$ são os autovalores de $D X(p)$. Para analisar a estrutura da singularidade, devemos estudar não só a variedade estável $\left(\lambda_{2}<0\right)$ ou instável $\left(\lambda_{2}>0\right)$ tangente ao auto-espaço associado a $\lambda_{2}$, mas também o comportamento da variedade 1-dimensional de classe $C^{r}$ tangente ao auto-espaço do autovalor nulo $\lambda_{1}$. A última variedade, invariante sob o fluxo de $X$, é chamada variedade central de $p$.

Para estudar as singularidades não-hiperbólicas, aplicamos uma mudança de variáveis nas direções $x$ e $y$ chamada de "blow-up". A mudança $x=$ $r \cos (\theta), y=r \sin (\theta)$ transforma o campo $X$ em um campo $\hat{X}$. Algumas vezes precisamos multiplicar $\hat{X}$ por uma potência de $r$ para reparametrizar o tempo. O campo $\bar{X}=r^{k} \hat{X}$ é chamado o "blow-up polar" sendo $k$ algum número inteiro. Existem também, os blow-ups direcionais. Estes são dados pelas mudanças de variáveis seguintes.

Na direção $x$ positiva, aplicamos $x=u$ e $y=u v$ obtendo o campo $\hat{X}_{x}$.

Na direção $y$ positiva, aplicamos $x=u v$ e $y=v$ obtendo o campo $\hat{X}_{y}$.

Da mesma maneira, conseguimos os blow-ups nas direções $x$ e $y$ negativas. Também se for necessário, multiplicamos $\hat{X}_{x}$ por $u^{k}$ e $\hat{X}_{y}$ por $v^{k}$ para reparametrizações do tempo, dando lugar aos campos

$$
\bar{X}_{x}=u^{k} \hat{X}_{x}, \bar{X}_{y}=v^{k} \hat{X}_{y}
$$

onde $k$ é um inteiro. Para maiores detalhes sobre o "blow-up" ver $[9,8]$.

Nas novas variáveis espera-se que o campo tenha singularidades elementares (hiperbólicas e semi-hiperbólicas). Caso contrário, aplica-se novas mudanças de variáveis para cada singularidade não-elementar. O retrato de fase 
de uma singularidade não-hiperbólica é obtido combinando os setores típicos determinados pelas singularidades elementares. Ver [8].

\subsection{2 Órbitas periódicas}

Dada $\mathcal{O}(\gamma, p)$ uma órbita de $X$ que passa por $p$ correspondente a trajetória $\gamma: \Re \rightarrow M$. A órbita chama-se periódica se existe um $\tau \in \Re$ tal que $\gamma(t+\tau, p)=\gamma(t, p), \forall t \in \Re$.

Para estudar o comportamento do campo numa vizinhança de uma órbita periódica $\mathcal{O}(\gamma, p)$, definimos uma aplicação entre duas seções transversais ao campo nos pontos $p$ e $p^{\prime} \in \mathcal{O}(\gamma, p)$ respectivamente.

Sejam $\Sigma$ uma subvariedade de $\Re^{2}$ e $p$ um ponto em $\Sigma \cap M$. Dizemos que $\Sigma$ é transversal ao campo em $p$, se $X(p)$ não está contido no espaço tangente a $\Sigma$ em $p$. Um subconjunto aberto $V$ de $\Sigma$ é transversal ao campo, se é transversal em cada ponto de $V$.

Sejam $\Sigma$ e $\Sigma^{\prime}$ duas seções transversais ao campo $X$ em $p$ e $p^{\prime}$ respectivamente com $p^{\prime} \in \mathcal{O}(\gamma, p)$. Existem vizinhanças de $p$ e $p^{\prime}, \Sigma_{0} \subset \Sigma$ e $\Sigma_{0}^{\prime} \subset \Sigma^{\prime}$ e existe uma aplicação $\xi: \Sigma_{0} \rightarrow \Re$ tal que $\gamma(\xi(q), q) \in \Sigma_{0}^{\prime}, \forall q \in \Sigma_{0}$. A função $\xi$ é da mesma classe que o campo. A aplicação $\pi: \Sigma_{0} \rightarrow \Sigma_{0}^{\prime}$ definida por $\pi(q)=\gamma(\xi(q), q)$ é chamada aplicação de Poincaré. Quando $\Sigma_{0}=\Sigma_{0}^{\prime}, \pi(q)$ é chamada aplicação do primeiro retorno.

O estudo da aplicação de Poincaré permite descrever o comportamento das órbitas vizinhas à órbita periódica. Em particular, as órbitas periódicas numa vizinhança de $\mathcal{O}(p, X)$ são pontos fixos da aplicação $\pi(q)$.

Uma órbita periódica $\mathcal{O}(\gamma, p)$ é dita ciclo limite, se possui uma vizinhança que não contem nenhuma órbita periódica distinta dela. Em outras palavras, dizemos que $p$ é um ponto fixo isolado da aplicação de Poincaré.

A seguinte proposição dá uma expressão da derivada da aplicação de retorno em termos das derivadas das funções componentes do campo.

Proposição 1.2 Sejam $M$ um aberto de $\Re^{2}$ e $X=(P(x, y), Q(x, y))$ um campo vetorial sobre $M$ de classe $C^{1}$. Seja $\mathcal{O}(\gamma, p)=\{\gamma(t, p): t \in \Re\}$ uma órbita periódica de $X$ de período $\tau$ e seja $\pi: \Sigma_{0} \rightarrow \Sigma_{0}$ a aplicação de Poincaré definida em uma seção transversal de $X$ em $p$. Então

$$
\pi^{\prime}(p)=\exp \left(\int_{0}^{\tau}\left(\frac{\partial}{\partial x} P(\gamma(t))+\frac{\partial}{\partial y} Q(\gamma(t))\right) d t\right) .
$$

O número

$$
\chi=\int_{0}^{\tau}\left(\frac{\partial}{\partial x} P(\gamma(t))+\frac{\partial}{\partial y} Q(\gamma(t))\right) d t
$$


permite determinar a estabilidade de uma órbita periódica isolada $\mathcal{O}(\gamma, p)=$ $\{\gamma(t, p): t \in \Re\}$.

Se $\chi<0, \mathcal{O}(\gamma, p)$ é um ciclo limite estável. Se $\chi>0, \mathcal{O}(\gamma, p)$ é um ciclo limite instável.

Se $X$ é analítico e a aplicação $\pi(q)$ é igual a $q$, para todo $q$ numa vizinhança da órbita $\mathcal{O}(\gamma, p)$, então $\mathcal{O}(\gamma, p)$ pertence ao interior de uma faixa de órbitas periódicas. Se $\pi(q)$ não é a identidade, podemos escrever $\pi(x)=x+a_{k}(x-p)^{k}+\cdots$ com $a_{k} \neq 0$. Dizemos, então, que o ciclo limite é

- estável se $k$ é ímpar e $a_{k}<0$ (as órbitas perto de $\mathcal{O}(\gamma, p)$ espiralam tendendo a $\mathcal{O}(\gamma, p))$

- instável se $k$ é ímpar e $a_{k}>0$ (as órbitas perto de $\mathcal{O}(\gamma, p)$ espiralam se afastando de $\mathcal{O}(\gamma, p))$;

- semi-estável se $k$ é par (as órbitas perto de $\mathcal{O}(\gamma, p)$ espiralam tendendo a (se afastando de) $\mathcal{O}(\gamma, p)$ pelo interior e afastando-se (tendendo a) pelo exterior da $\mathcal{O}(\gamma, p))$.

\subsection{Compactificação}

Um processo de compactificação de um campo vetorial $X$ consiste em aplicar uma mudança de variáveis que transforme o espaço de fase $M$, uma superfície aberta, em uma superfície compacta $\widetilde{M}$ e o campo $X$ definido em $M$ em um campo $\widetilde{X}$ sobre $\widetilde{M}$. Em geral, é preciso multiplicar por uma função adequada $\rho$ tal que o campo $\rho \widetilde{X}$ se estenda analiticamente a todo $\widetilde{M}$. Então, é possível obter um conhecimento do comportamento das órbitas de $X$ numa vizinhança do infinito.

Apresentamos, a seguir, uma compactificação que chamaremos de compactificação cilíndrica e uma transformação local das vizinhanças do infinito de $M$. Todas elas permitirão determinar o comportamento das equações de segunda ordem, $E_{f}$, no infinito. Destacamos que as transformações são dadas com preferência na direção vertical do cilindro $M$ pelo fato do crescimento das trajetórias dos campos associados $X(f)$ acontecer só nessa direção.

\subsubsection{Compactificação cilíndrica}

A mudança de variável $u=x, v=\arctan (y)$ transforma $M=S^{1} \times \Re$ no cilindro compacto $\widetilde{M}=S^{1} \times\left[-\frac{\pi}{2},+\frac{\pi}{2}\right]$. O infinito de $M$ é transformado na fronteira de $\widetilde{M}$ que denotamos pelos círculos $C_{-\frac{\pi}{2}}=S^{1} \times\left\{-\frac{\pi}{2}\right\}$ e $C_{+\frac{\pi}{2}}=$ 
$S^{1} \times\left\{+\frac{\pi}{2}\right\}$. Ver figura 1.1. O campo $X(x, y)=(P(x, y), Q(x, y))$ sobre $M$ é transformado no campo

$$
\widetilde{X}_{1}(u, v)=\left(P(u, \tan (v)), \cos ^{2}(v) Q(u, \tan (v))\right) .
$$
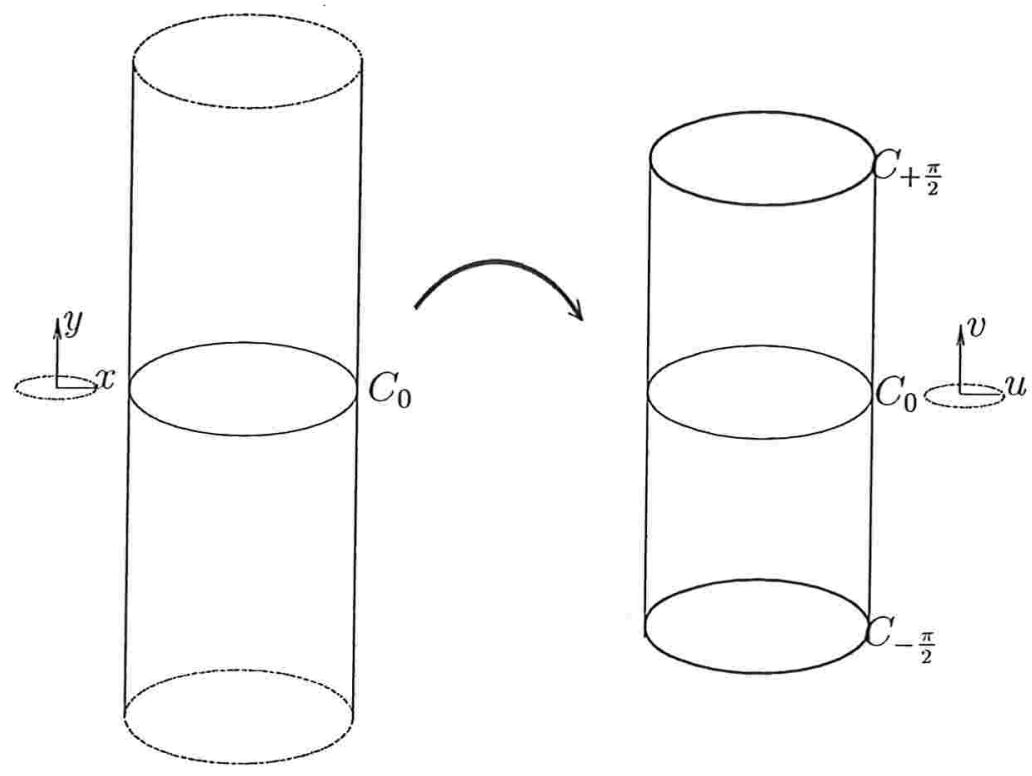

Figura 1.1: Compactificação cilíndrica dada pela transformação $u=x \mathrm{e}$ $v=\arctan (y)$

Para conseguir o campo $\widetilde{X}_{1}$ bem definido em todo $\widetilde{M}$, devemos multiplicar pela função $\cos ^{k}(v)$ para algum $k$ inteiro positivo. Isto será feito para os campos $X(f)$ no capítulo 2 .

\subsubsection{Transformação das vizinhanças do infinito de $M$}

A mudança de variável $x=u$ e $y=\frac{1}{v}$ para $v \neq 0$ transforma uma vizinhança do $\pm \infty$ de $M=S^{1} \times \Re$ em conjuntos semi-abertos. Isto é, as vizinhanças do $\pm \infty$

$$
U_{r}^{+}=\left\{(x, y) \in M \mid x \in S^{1}, y>r\right\}
$$

e

$$
U_{r}^{-}=\left\{(x, y) \in M \mid x \in S^{1}, y<-r\right\}
$$

onde $r \in \Re^{+}$são transformados nos conjuntos

$$
\widetilde{U}_{r}^{+}=\left\{(x, y) \in M \mid x \in S^{1}, 0 \leq y<r^{-1}\right\}
$$


$\mathrm{e}$

$$
\widetilde{U}_{r}^{-}=\left\{(x, y) \in M \mid x \in S^{1},-r^{-1}<y \leq 0\right\}
$$

respectivamente. O infinito é transformado no círculo $\widetilde{C}_{0}=S^{1} \times\{0\}$. Ver figura 1.2. O campo $X(x, y)=(P(x, y), Q(x, y))$ sobre $M$ é transformado no campo

$$
\widetilde{X}_{2}(u, v)=\left(P\left(u, \frac{1}{v}\right),-v^{2} Q\left(u, \frac{1}{v}\right)\right) .
$$

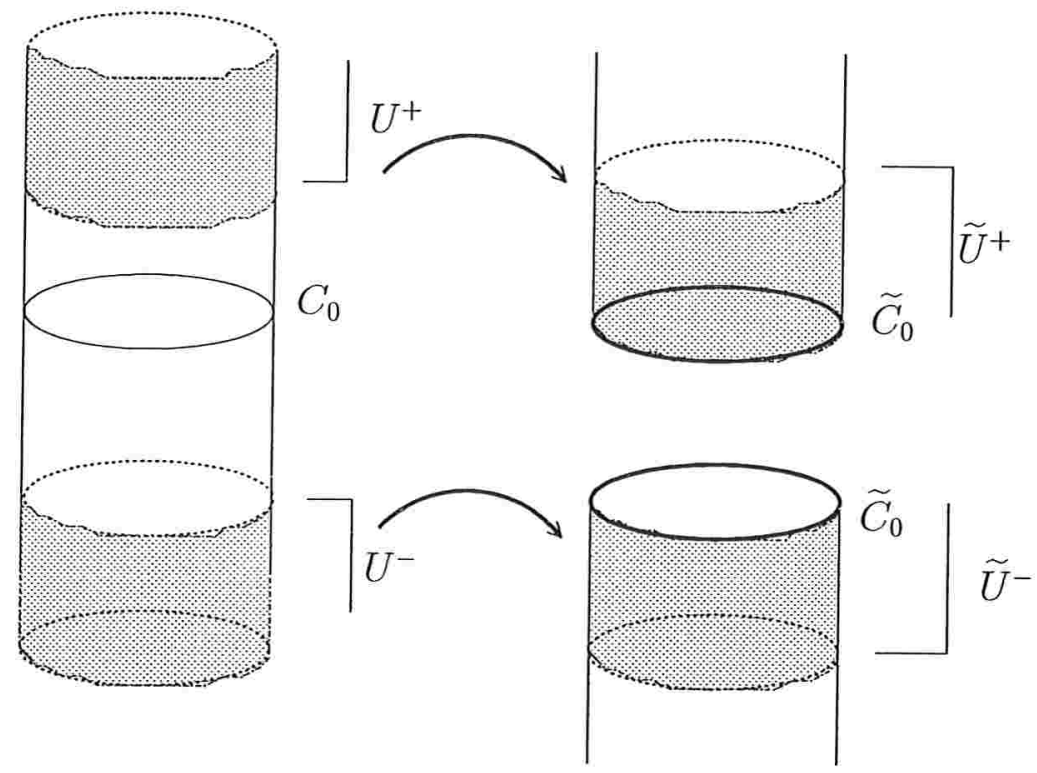

Figura 1.2: Transformação de vizinhança do infinito de $S^{1} \times \Re$ pela mudança de variável $u=x$ e $v=\frac{1}{y}$

Da mesma maneira que na compactificação anterior, precisamos multiplicar pela função $v^{k}$ com $k$ inteiro apropriado para obtermos uma boa definição de $\widetilde{X}_{2}$ em vizinhanças abertas de $\widetilde{U}^{ \pm}$.

No capítulo 2 , veremos que os campos vetoriais $X(f)$ com as funções componentes $P(x, y)=y$ e $Q(x, y)=\sum_{i=0}^{n} a_{i}(x) y^{i}$ podem ser transformados por estas compactificações e estendidos a campos $C^{r}$ em todo $\widetilde{M}$, para $r \geq 1$.

\subsection{Comportamento qualitativo no infinito}

Para estudar a estabilidade estrutural dos campos vetoriais sobre superfícies abertas sem transformar o espaço de fase nem o campo (i.e. sem nenhuma compactificação), precisa-se definir comportamentos particulares das órbitas 
não-limitadas numa vizinhança do infinito. Ver $[15,6,14]$ como exemplo deste enfoque.

Em razão dos conjuntos $\alpha$ e $\omega$-limite de órbitas que escapam ao infinito serem vazios, introduz-se o conceito de conjuntos limites prolongados (positivo e negativo). Estes conjuntos são definidos para $p \in M$ por

$$
J^{ \pm}(p, X(f))=\left\{q \in M \mid \exists t_{n} \rightarrow \pm \infty, p_{n} \rightarrow p \text { tais que } \gamma\left(t_{n}, p_{n}\right) \rightarrow q\right\} .
$$

Define-se, então, um comportamento do campo $X$ perto do infinito como segue.

Definição 1.1 Duas semi-órbitas $\mathcal{O}^{+}(\gamma, p)$ e $\mathcal{O}^{-}(\gamma, q)$ que passam por $p$ e $q$ respectivamente formam uma "sela no infinito" se

$\omega\left(\mathcal{O}^{+}(\gamma, p)\right)=\emptyset, \alpha\left(\mathcal{O}^{+}(\gamma, p)\right)=\emptyset$ e $q \in J^{+}(p, X)$ (ou equivalentemente $\left.p \in J^{-}(q, X)\right)$.

A semi-órbita $\mathcal{O}^{+}(p, X(f))$ é chamada de separatriz estável e $\mathcal{O}^{-}(q, X(f))$ de separatriz instável.

Em geral, valem as relações $\omega(p, X(f)) \subset J^{+}(p, X(f))$, e $\alpha(p, X(f)) \subset$ $J^{-}(p, X(f))$.

Em [14], modificou-se o espaço de fase acrescentando um conjunto de pontos chamados finais ou infinitos. Nós pensamos que esse fato é na verdade um tipo de compactificação, utilizado para dar um entendimento um pouco melhor das trajetórias perto do infinito. Mas, ainda se continua detectando só o comportamento de tipo "sela no infinito".

A aplicação de uma compactificação como as estudadas na seção 1.3, oferece maiores evidências sobre o comportamento das órbitas numa vizinhança do infinito. De fato, definimos as singularidades de $X(f)$ no infinito de $M=S^{1} \times \Re$ como as singularidades ou tangências de $\widetilde{X}_{1}(f)$ (ver definição na seção 1.3.1) na fronteira de $\widetilde{M}=S^{1} \times\left[-\frac{\pi}{2}, \frac{\pi}{2}\right]$. As singularidades ou tangências de $\widetilde{X}_{1}(f)$ serão estudadas com maiores detalhes no Capítulo 2.

Da mesma maneira, podemos definir as singularidades de $X(f)$ no infinito como as singularidades ou tangências de $\widetilde{X}_{2}(f)$ (ver definição na seção 1.3.2) no círculo $\widetilde{C}_{0}=S^{1} \times\{0\}$.

Portanto, uma "sela no infinito" de $X$ não é mais que uma singularidade ou uma tangência de $\widetilde{X}_{i}(f)$ para $i=1,2$ nos círculos $C_{ \pm \frac{\pi}{2}}$ e $\widetilde{C}_{0}$, com duas órbitas formando um setor hiperbólico. Mais especificamente, dizemos que se duas semi-órbitas $\mathcal{O}^{+}(\gamma, p)$ e $\mathcal{O}^{-}(\gamma, q)$ formam uma "sela no infinito" para $X$, então existe $p^{*} \in \partial \widetilde{M}_{i}$ tal que $\omega\left(\mathcal{O}^{+}\left(\tilde{p}, \widetilde{X}_{i}\right)\right)=\left\{p^{*}\right\}$ e $\alpha\left(\mathcal{O}^{-}\left(\tilde{q}, \widetilde{X}_{i}\right)\right)=\left\{p^{*}\right\}$ 
e as órbitas $\mathcal{O}^{+}\left(\tilde{p}, \widetilde{X}_{i}\right)$ e $\mathcal{O}^{-}\left(\tilde{q}, \widetilde{X}_{i}\right)$ determinam um setor hiperbólico onde $\tilde{p}$ e $\tilde{q} \in \widetilde{M}_{i}$ são as transformações de $p$ e $q$ respectivamente.

Com as representações $\widetilde{X}_{1}$ em $\widetilde{M}$ ou $\widetilde{X}_{2}$ em $\widetilde{U}$, outros comportamentos são detectados perto do infinito de $M$. Como veremos no Capítulo 2, no infinito de $M$ encontramos órbitas periódicas e singularidades além da "sela no infinito" apresentada acima. Observamos que se $\partial \widetilde{M}$ (ou melhor dito $C_{ \pm \frac{\pi}{2}}$ ou $\widetilde{C}_{0}$ ) forma uma órbita periódica para $\tilde{X}(f)$ então $X(f)$ não tem órbitas que tendem ao infinito formando uma "sela no infinito".

\subsection{Estabilidade Estrutural}

Nesta seção, apresentamos os conceitos básicos da estabilidade estrutural de campos vetoriais sobre uma região compacta do plano com bordo suave.

Lembramos que estamos interessados na estabilidade estrutural de campos vetoriais definidos no cilindro $M=S^{1} \times \Re$ que provêm das equações diferenciais de segunda ordem. Neste caso, o espaço de fase $M$ é uma superfície aberta. Então, uma compactificação nos permite estudar a estabilidade estrutural desses campos vetoriais, agora definidos sobre uma região compacta com bordo. E ainda mais, admitimos que o bordo contenha singularidades ou constitua uma órbita periódica.

Denotamos por $\mathcal{E}^{n, r}(M)$ (ou simplesmente $\mathcal{E}^{n, r}$ ) o conjunto de campos vetoriais $X(f)$ da forma $X(f)=y \frac{\partial}{\partial x}+f(x, y) \frac{\partial}{\partial y}$ onde $f(x, y)=\sum_{i=0}^{n} a_{i}(x) y^{i}$ é um polinômio em $y$ de grau $n$ fixo, cujos coeficientes $a_{i}: \Re \rightarrow \Re$ são funções periódicas em $x$ de classe $C^{r} \operatorname{com} r \geq 1$.

Em toda esta seção, $\widetilde{X}(f)$ denotará a compactificação de $X(f)$ do tipo estudada na seção 1.3 .1 e $\widetilde{M}$ a compactificação de $M$.

Definição 1.2 A distância entre os campos vetoriais

$$
X(f)=X\left(\sum_{i=0}^{n} a_{i}(x) y^{i}\right) \text { e } X(g)=X\left(\sum_{i=0}^{n} b_{i}(x) y^{i}\right) \in \mathcal{E}^{n, r}(M) \text { é definida }
$$
por

$$
|X(f)-X(g)|=\sup _{0 \leq k \leq r}\left\{\left|\frac{d^{k}}{d x^{k}}\left(a_{i}(x)-b_{i}(x)\right)\right|, x \in \Re\right\}
$$

Dizemos que $X(g)$ está $\epsilon$-próximo de $X(f)$ se $|X(f)-X(g)|<\epsilon$. Segue imediatamente que $\widetilde{X}(g)$ está $\epsilon$-próximo de $\widetilde{X}(f)$ em $\widetilde{M}$.

Damos a seguir uma definição de estabilidade estrutural dos campos $X(f)$ sobre $M$ que contemple de maneira natural o comportamento das trajetórias no infinito. 
Definição 1.3 Um campo $X(f) \in \mathcal{E}^{n, r}(M)$ é estruturalmente estável em $M$ se existe uma vizinhança $\mathcal{U}$ de $X(f)$ tal que $\forall X(g) \in \mathcal{U}$, existe um homeomorfismo $h_{g}: \widetilde{M} \rightarrow \widetilde{M}$ que leva trajetórias de $\widetilde{X}(f)$ em trajetórias de $\widetilde{X}(g)$.

A estabilidade estrutural de campos vetoriais tem dois aspectos principais a serem tratados. Um aspecto é determinar os campos que tenham propriedades simples e genéricas, isto é determinar um conjunto $\Sigma^{n}$ que seja aberto e denso em $\mathcal{E}^{n, r}$. Outro aspecto é provar que os campos de $\Sigma^{n}$ caracterizam os campos estruturalmente estáveis.

Para determinar a densidade, provaremos que a medida do conjunto complementar de $\Sigma^{n}$ em $\mathcal{E}^{n, r}$ tem medida de Lebesgue zero.

O seguinte lema será de muita utilidade na determinação da densidade de $\Sigma^{n}$. Corresponde ao caso mais simples do Teorema de Sard, referente à medida de Lebesgue do conjunto de valores críticos de uma aplicação $g$ : $M \rightarrow N$ de classe $C^{r}$ onde $M$ e $N$ são variedades diferenciáveis.

Lema 1.1 (Sard) Seja $g: I \rightarrow \Re$ uma função de classe $C^{1}$ definida em um subconjunto aberto $I$ de $\Re$. O conjunto de valores críticos de $g$ dado por

$$
\operatorname{Crit}(g)=\left\{y=g(x): g^{\prime}(x)=0\right\}
$$

tem medida de Lebesgue zero em $\Re$.

Encontramos uma prova do Teorema de Sard em [11].

Lembramos que um ponto $p \in M$ é dito ponto crítico de uma aplicação $g: M \rightarrow N$ de classe $C^{1}$, se o co-posto $(D g)_{p}=\min \{\operatorname{dim}(M), \operatorname{dim}(N)\}-$ posto $(D g)_{p}>0$. O valor $g(p)$ é chamado de valor crítico.

Para caracterizar os campos estruturalmente estáveis, precisamos construir um homeomorfismo de $\widetilde{M}$ em si mesmo que transforme trajetórias de um campo $\widetilde{X}(f)$ em trajetórias dos campos suficientemente próximos. Para isso, seguindo os métodos utilizados nos trabalhos [18, 19], dividimos o espaço de fase $\widetilde{M}$ em setores conexos cujas fronteiras estão formadas por trajetórias chamadas de separatrizes. Agora, definimos mais precisamente.

Definição 1.4 Uma separatriz de $X(f)$ é um conjunto invariante dos seguintes tipos:

a) uma singularidade de $\widetilde{X}(f)$ em $\widetilde{M}$ (pode pertencer a $\partial \widetilde{M})$.

b) um ponto de tangência externa de uma trajetória de $\widetilde{X}(f)$ com $\partial \widetilde{M}$.

c) uma trajetória contida no interior de $\widetilde{M}$ tangente a $\partial \widetilde{M}$. 
d) um ciclo limite contido no interior de $\widetilde{M}$.

e) uma trajetória contida no interior de $\widetilde{M}$ que tende a uma sela contida no interior de $\widetilde{M}$.

f) uma trajetória contida no interior de $\widetilde{M}$ que tende a uma singularidade de $\widetilde{X}(f)$ pertencente a $\partial \widetilde{M}$. A trajetória é uma variedade invariante da singularidade. No caso de um nó, considera-se a variedade fraca.

Definição 1.5 Uma região canônica de $\widetilde{X}(f)$ é uma componente conexa de $\widetilde{M}-W$ onde $W$ é o conjunto de todas as separatrizes de $\widetilde{X}(f)$. 


\section{Capítulo 2}

\section{Campos de vetores $X(f)$ associados as equações de segunda ordem}

Neste capítulo, descrevemos o comportamento do campo $X(f)=y \frac{\partial}{\partial x}+$ $f(x, y) \frac{\partial}{\partial y}$ associado à equação diferencial de segunda ordem $E_{f}: x^{\prime \prime}=f\left(x, x^{\prime}\right)$ onde $f(x, y)=\sum_{i=0}^{n} a_{i}(x) y^{i}$ e $a_{i}(x)$ são funções periódicas de classe $C^{r}, r \geq 1$ e $n \geq 1$.

Denotamos por $\mathcal{E}^{n, r}(M)$ o espaço de campos $X(f)$ definidos em $M=$ $S^{1} \times \Re$.

Descrevemos o comportamento do campo $X(f)$ em uma vizinhança do infinito de $M$ através das compactificações introduzidas na seção 1.3. Denotamos por $\widetilde{M}$ e $\widetilde{X}(f)$ a compactificação de $M$ e $X(f)$ respectivamente.

\subsection{Comportamento das trajetórias de $X(f)$}

As características mais relevantes do campo $X(f)$ em $M=S^{1} \times \Re$ são:

1. Na parte superior do cilindro $M$, as órbitas de $X(f)$ são percorridas da esquerda para a direita, enquanto que na parte inferior vão da direita para a esquerda considerando valores crescentes do tempo.

2. No círculo $C_{0}=S^{1} \times\{0\} \subset M$, o campo é vertical em todo ponto não-singular.

3. As singularidades ficam só sobre o eixo $x$. Se $X(f)$ tem uma singularidade do tipo sela hiperbólica, então deve existir uma outra singu- 
laridade com índice +1 . Isto segue de observar o comportamento das trajetórias perto de uma singularidade. Ver figura 2.1.

4. As trajetórias podem crescer ilimitadamente sobre o eixo $y$.

5. $X(f)$ pode ter dois tipos de órbitas periódicas na parte finita de $M$. As homotópicas a zero e as não-homotópicas a zero. As primeiras contêm em seu interior uma singularidade e as últimas circundam o cilindro sem cortar o círculo $C_{0}$.

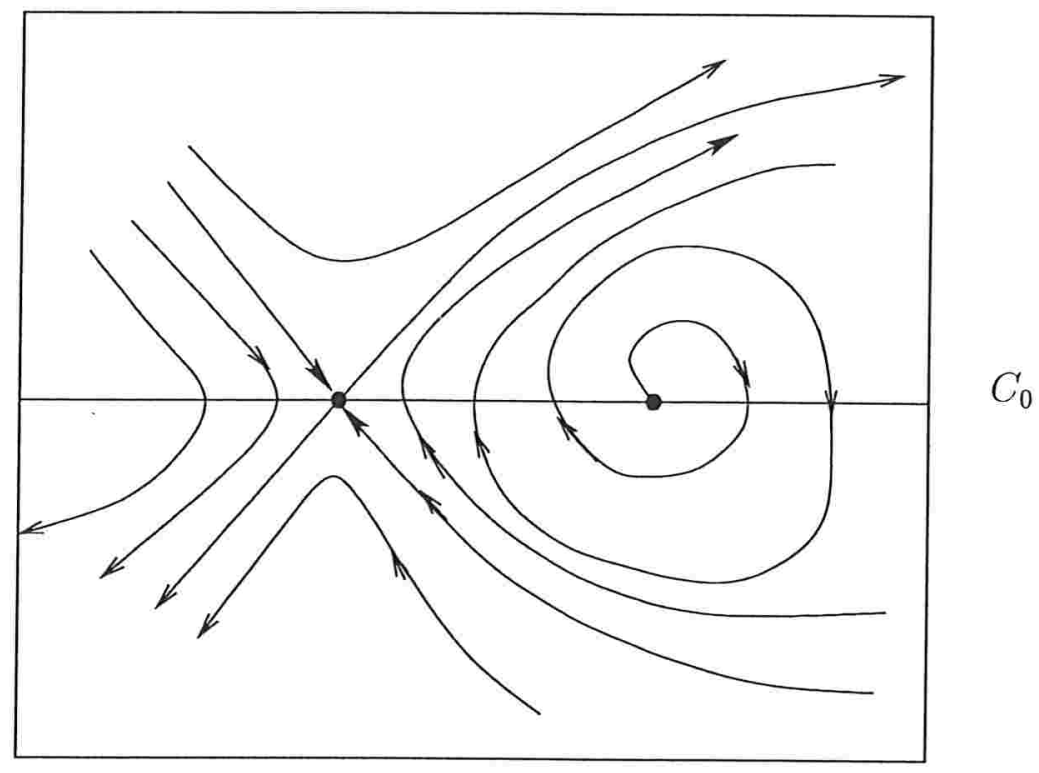

Figura 2.1: Orientação das trajetórias numa vizinhança de uma sela e de um foco

\subsubsection{Singularidades}

$\mathrm{Na}$ seguinte proposição, apresentamos as propriedades das singularidades elementares do campo $X(f)$ em termos das funções coeficientes.

Proposição 2.1 Dado $X(f) \in \mathcal{E}^{n, r}(M), \operatorname{com} f(x, y)=\sum_{i=0}^{n} a_{i}(x) y^{i}$. Então, as singularidades de $X(f)$ são da forma $\left(x_{*}, 0\right)$ onde $x_{*}$ é um zero de $a_{0}(x)=$ 0. O tipo de $\left(x_{*}, 0\right)$ é:

a) sela se $a_{0}^{\prime}\left(x_{*}\right)>0$, 
b) nó se $a_{0}^{\prime}\left(x_{*}\right)<0$ e $a_{1}^{2}\left(x_{*}\right)+4 a_{0}^{\prime}\left(x_{*}\right)>0$,

c) foco se $a_{0}^{\prime}\left(x_{*}\right)<0$ e $a_{1}^{2}\left(x_{*}\right)+4 a_{0}^{\prime}\left(x_{*}\right)<0$,

d) centro se $a_{0}^{\prime}\left(x_{*}\right)<0$ e $a_{1}\left(x_{*}\right)=0$.

Mais ainda, os espaços próprios associados aos autovalores de $D X(f)$ nunca são horizontais.

Prova. A matriz jacobiana de $X(f)$ avaliada na singularidade $\left(x_{*}, 0\right)$ tem a forma

$$
D X(f)=\left(\begin{array}{cc}
0 & 1 \\
a_{0}^{\prime}\left(x_{*}\right) & a_{1}\left(x_{*}\right)
\end{array}\right),
$$

com os autovalores $\lambda_{ \pm}=\frac{-a_{1}\left(x_{*}\right) \pm \sqrt{a_{1}^{2}\left(x_{*}\right)+4 a_{0}^{\prime}\left(x_{*}\right)}}{2}$ e os autovetores associados $v_{ \pm}=\left(1, \lambda_{ \pm}\right)$. As conclusões da proposição seguem se analisarmos os sinais de $\lambda_{ \pm}$em termos dos coeficientes $a_{i}(x)$. Os tipos topológicos são mostrados na figura 2.2

a)
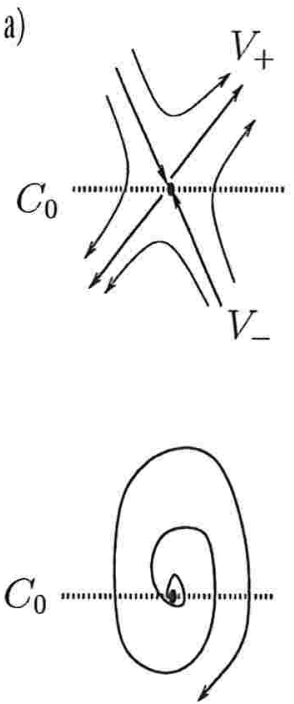
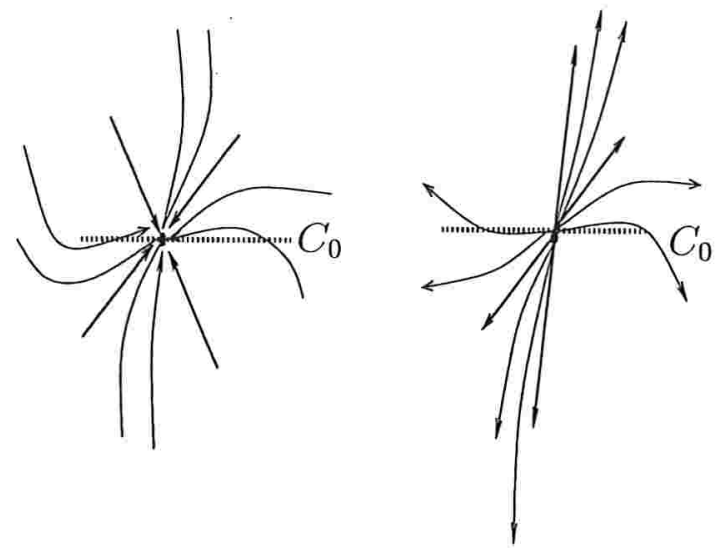

Figura 2.2: Tipos topológicos de singularidades no círculo $C_{0}$

\subsection{Compactificação de $X(f)$}

Nesta seção, apresentamos as expressões do campo $X(f)$ onde $f(x, y)=$ $\sum_{i=0}^{n} a_{i}(x) y^{i}$ e as funções periódicas $a_{i}: \Re \rightarrow \Re$ de classe $C^{r}$ com $r \geq 1$, segundo as compactificações introduzidas na seção 1.3. 


\subsubsection{Compactificação cilíndrica}

Compactificamos o espaço de fase $M$ pela mudança de variáveis $u=x$ e $v=\arctan (y)$ obtendo o espaço $\widetilde{M}=S^{1} \times\left[-\frac{\pi}{2}, \frac{\pi}{2}\right]$. Notaremos por $C_{0}$ e $C_{ \pm \frac{\pi}{2}}$ os círculos $S^{1} \times\{0\}$ e $S^{1} \times\left\{ \pm \frac{\pi}{2}\right\}$ respectivamente.

Descrevemos a seguir o campo $X(f)$ nas novas variáveis.

Proposição $2.2 \operatorname{Seja} X(f) \in \mathcal{E}^{n, r}(M) \operatorname{com} f(x, y)=\sum_{i=0}^{n} a_{i}(x) y^{i}$. Então para $n=1$,

$$
\widetilde{X}_{1}(f)=\operatorname{sen}(v) \frac{\partial}{\partial u}+\left(a_{0}(u) \cos ^{3}(v)+a_{1}(u) \operatorname{sen}(v) \cos ^{2}(v)\right) \frac{\partial}{\partial v}
$$

para $n=2$,

$$
\begin{aligned}
\tilde{X}_{1}(f)= & \operatorname{sen}(v) \frac{\partial}{\partial u}+\left(a_{0}(u) \cos ^{3}(v)+a_{1}(u) \operatorname{sen}(v) \cos ^{2}(v)\right. \\
& \left.+a_{2}(u) \operatorname{sen}^{2}(v) \cos (v)\right) \frac{\partial}{\partial v}
\end{aligned}
$$

para $n \geq 3$,

$$
\tilde{X}_{1}(f)=\operatorname{sen}(v) \cos ^{n-3}(v) \frac{\partial}{\partial u}+\left(\sum_{i=0}^{n} a_{i}(u) \operatorname{sen}^{i}(v) \cos ^{n-i}(v)\right) \frac{\partial}{\partial v}
$$

está bem definido e é da classe de diferenciabilidade de $a_{i}$ em $\widetilde{M}=S^{1} \times$ $\left[-\frac{\pi}{2}, \frac{\pi}{2}\right]$.

Prova. Aplicando ao campo $X(f)$ a mudança de variáveis $u=x$ e $v=\arctan (y)$, obtemos o campo definido em $(u, v) \in \widetilde{M^{\circ}}=S^{1} \times\left(-\frac{\pi}{2}, \frac{\pi}{2}\right)$ da seguinte forma

$$
Y(f)\left\{\begin{aligned}
u^{\prime} & =\frac{\operatorname{sen}(v)}{\cos (v)} \\
v^{\prime} & =\sum_{i=0}^{n} a_{i}(u) \operatorname{sen}^{i}(v) \cos ^{-i+2}(v)
\end{aligned}\right.
$$

Para obter um campo definido em todo $\widetilde{M}$, precisamos multiplicar por $\cos (v) \operatorname{nos} \operatorname{casos} n=1,2$, e por $\cos ^{n-2}(v)$ no caso $n \geq 3$. Em ambas situações, obtemos o campo $\widetilde{X}_{1}(f)$, bem definido em uma vizinhança do $\widetilde{M}$ e da classe de diferenciabilidade das funções $a_{i}$. 


\subsubsection{Uma representação local de $X(f)$ no infinito}

Aplicaremos a mudança de variáveis $u=x$ e $v=\frac{1}{y}$, para obter uma representação do campo $X(f)$ em vizinhanças $U_{r}^{+}$e $U_{r}^{-}$do infinito de $M=S^{1} \times \Re$ com $r>0$. Lembramos que $U_{r}^{+}$e $U_{r}^{-}$são transformados nos conjuntos $\widetilde{U}_{r}^{+}=\left\{(x, y) \in M \mid x \in S^{1}, 0 \leq y<r^{-1}\right\}$ e $\widetilde{U}_{r}^{-}=\left\{(x, y) \in M \mid x \in S^{1},-r^{-1}<\right.$ $y \leq 0\}$ respectivamente, e o infinito de $M$ é levado no círculo $\widetilde{C}_{0}=S^{1} \times\{0\}$.

Proposição 2.3 Seja $X(f) \in \mathcal{E}^{n, r}(M) \operatorname{com} f(x, y)=\sum_{i=0}^{n} a_{i}(x) y^{i}$. Então

$$
\begin{aligned}
& \text { para } n=1, \widetilde{X}_{2}(f)=\frac{\partial}{\partial u}-\left(a_{0}(u) v^{3}+a_{1}(u) v^{2}\right) \frac{\partial}{\partial v} \\
& \text { para } n=2, \widetilde{X}_{2}(f)=\frac{\partial}{\partial u}-\left(a_{0}(u) v^{3}+a_{1}(u) v^{2}+a_{2}(u) v\right) \frac{\partial}{\partial v} \\
& \text { para } n=3, \widetilde{X}_{2}(f)=\frac{\partial}{\partial u}-\left(a_{0}(u) v^{3}+a_{1}(u) v^{2}+a_{2}(u) v+a_{3}(u)\right) \frac{\partial}{\partial v} \\
& \text { para } n \geq 4, \widetilde{X}_{2}(f)=v^{n-3} \frac{\partial}{\partial u}-\sum_{i=0}^{n} a_{i}(u) v^{n-i} \frac{\partial}{\partial v} .
\end{aligned}
$$

Em todos os casos, $\widetilde{X}_{2}(f)$ está bem definido e é da classe de diferenciabilidade de $a_{i}$ em $\widetilde{U}_{r}^{ \pm}$.

Prova. Aplicando ao campo $X(f)$ a mudança de variáveis $x=u$ e $y=\frac{1}{v}$, obtemos o campo $Y(f)$ definido em $(u, v) \in \widetilde{M^{\circ}}=S^{1} \times(0, \infty)$ da seguinte forma.

$$
Y(f)\left\{\begin{aligned}
u^{\prime} & =\frac{1}{v} \\
v^{\prime} & =-v^{2} \sum_{i=0}^{n} a_{i}(u)\left(\frac{1}{v}\right)^{i}
\end{aligned}\right.
$$

Para obter um campo definido no círculo $\widetilde{C}_{0}$, precisamos multiplicar por $v$ nos $\operatorname{casos} n=1,2,3$ e por $v^{n-2}$ no caso $n \geq 4$. Em ambas situações, obtemos o campo $\widetilde{X}_{2}(f)$ requerido, isto é, bem definido em $\widetilde{U}_{r}^{ \pm}$e da classe de diferenciabilidade das funções $a_{i}$.

\subsection{Comportamento das órbitas de $\widetilde{X}(f)$}

Estamos interessados nas trajetórias de $X(f)$ perto do infinito. Para isso, estudamos os campos $\widetilde{X}_{1}(f)$ e $\widetilde{X}_{2}(f)$ em vizinhanças abertas dos círculos $C_{ \pm \frac{\pi}{2}}$ e $\widetilde{C}_{0}$ respectivamente.

As seguintes proposições descrevem o comportamento das órbitas de $\tilde{X}_{1}(f)$ numa vizinhança de $C_{ \pm \frac{\pi}{2}}$. Destacamos, também, que as mesmas proposições são válidas para a representação $\widetilde{X}_{2}(f)$ sobre o círculo $\widetilde{C}_{0}$.

Proposição 2.4 Seja $X(f) \in \mathcal{E}^{n, r}(M)$ onde $f(x, y)=\sum_{i=0}^{n} a_{i}(x) y^{i}$ de classe $C^{r}, r \geq 2$ e $n=1,2$. Então 
a) Para $n=1$, os círculos $C_{ \pm \frac{\pi}{2}}$ são órbitas periódicas de período $\tau$ com a primeira e a segunda derivadas da aplicação de Poincaré iguais a $1 e$ $\pm 2 \int_{0}^{\tau} a_{1}(s) d s$ respectivamente.

b) Para $n=2$, os círculos $C_{ \pm \frac{\pi}{2}}$ são órbitas periódicas de período $\tau$ hiperbólicas com $\chi=\mp \int_{0}^{\tau} a_{2}(s) d s$.

Prova. a) Denotamos por $\gamma_{ \pm}(u)=\left( \pm u, \pm \frac{\pi}{2}\right)$ as órbitas periódicas de $\widetilde{X}(f)$ nos círculos $C_{ \pm \frac{\pi}{2}}$, onde $u \in[0, \tau]$ e $\tau$ é o período das funções $a_{i}(u)$. Tomando a mudança de variáveis $u=s$ e $v= \pm \frac{\pi}{2}-\eta$, o campo $\widetilde{X}_{1}(f)$ tem associado as equações diferenciais da seguinte forma

$$
\left\{\begin{array}{l}
\frac{d s}{d t}= \pm \cos (\eta) \\
\frac{d \eta}{d t}=a_{0}(s) \sin ^{3}(\eta) \pm a_{1}(s) \sin ^{2}(\eta) \cos (\eta)
\end{array}\right.
$$

Se dividimos a segunda equação pela primeira, obtemos a equação diferencial

$$
\frac{d \eta}{d s}=R(s, \eta)
$$

onde $R(s, \eta)= \pm a_{0}(s) \frac{\sin ^{3}(\eta)}{\cos (\eta)}+a_{1}(s) \sin ^{2}(\eta)$ e $R(s, 0)=0$

Seja $\eta=f\left(s ; s_{0}, \eta_{0}\right)$ solução da equação (2.2) com condição inicial

$$
f\left(s_{0} ; s_{0}, \eta_{0}\right)=\eta_{0} .
$$

Podemos considerar, sem perda de generalidade, $s_{0}=0$.

A função do primeiro retorno é dada por $\phi\left(\eta_{0}\right)=f\left(\tau ; 0, \eta_{0}\right)$. Esta função está definida sobre um arco sem contato normal a órbita periódica $\gamma_{ \pm}$.

Consideramos a função $d\left(\eta_{0}\right)=\phi\left(\eta_{0}\right)-\eta_{0}$.

Estamos interessados nas derivadas primeira e segunda da função $d\left(\eta_{0}\right)$.

Tendo em conta a relação

$$
\frac{d \eta}{d s}=R\left(s, f\left(s ; 0, \eta_{0}\right)=\frac{d}{d s} f\left(s ; 0, \eta_{0}\right),\right.
$$

obtemos as equações diferenciais

$$
\begin{aligned}
\frac{d}{d s}\left(\frac{\partial f}{\partial \eta_{0}}\right)= & \frac{\partial}{\partial \eta} R\left(s, f\left(s ; 0, \eta_{0}\right)\right) \cdot \frac{\partial f}{\partial \eta_{0}}\left(s ; 0, \eta_{0}\right) \\
\frac{d}{d s}\left(\frac{\partial^{2} f}{\partial \eta_{0}^{2}}\right)= & \frac{\partial^{2}}{\partial \eta^{2}} R\left(s, f\left(s ; 0, \eta_{0}\right)\right) \cdot\left(\frac{\partial f}{\partial \eta_{0}}\left(s ; 0, \eta_{0}\right)\right)^{2} \\
& +\frac{\partial}{\partial \eta} R\left(s, f\left(s ; 0, \eta_{0}\right)\right) \frac{\partial^{2} f}{\partial \eta_{0}^{2}}\left(s ; 0, \eta_{0}\right)
\end{aligned}
$$


As soluções das equações (2.3) e (2.4) são

$$
\begin{aligned}
\frac{\partial f}{\partial \eta_{0}}\left(s ; 0, \eta_{0}\right)= & \exp \left(\int_{0}^{s} \frac{\partial}{\partial \eta} R\left(t, f\left(t ; 0, \eta_{0}\right)\right) d t\right) \\
\frac{\partial^{2} f}{\partial \eta_{0}^{2}}\left(s ; 0, \eta_{0}\right)= & \exp \left(\int_{0}^{s} \frac{\partial}{\partial \eta} R\left(t, f\left(t ; 0, \eta_{0}\right)\right) d t\right) \\
& \cdot \int_{0}^{s} \frac{\partial^{2}}{\partial \eta^{2}} R\left(t, f\left(t ; 0, \eta_{0}\right)\right) \\
& \cdot\left(\frac{\partial f}{\partial \eta_{0}}\left(t ; 0, \eta_{0}\right)\right)^{2} \cdot \exp \left(-\int_{0}^{t} \frac{\partial}{\partial \eta} R\left(\tilde{t}, f\left(t ; 0, \eta_{0}\right)\right) d \tilde{t}\right) d t
\end{aligned}
$$

Calculamos as derivadas parciais de $R(s, \eta)$ respeito de $\eta$.

$$
\begin{aligned}
\frac{\partial R}{\partial \eta}(s, \eta)= \pm a_{0}(s) & \left(3 \sin ^{2}(\eta)+\frac{\sin ^{4}(\eta)}{\cos ^{2}(\eta)}\right)+2 a_{1}(s) \sin (\eta) \cos (\eta) \\
\frac{\partial^{2} R}{\partial \eta^{2}}(s, \eta)= & \pm a_{0}(s)\left(6 \frac{\sin (\eta)}{\cos ^{3}(\eta)}+4 \frac{\sin ^{3}(\eta)}{\cos (\eta)}\right. \\
& \left.+2 \frac{\sin ^{5}(\eta)}{\cos ^{3}(\eta)}\right)+2 a_{1}(s)\left(\cos ^{2}(\eta)-\sin (\eta)\right) .
\end{aligned}
$$

Para $\eta_{0}=0$ e $f\left(s ; 0, \eta_{0}\right)=\eta_{0}$, segue de (2.5), (2.6), (2.7) e (2.8), as expressões

$$
\begin{gathered}
d\left(\eta_{0}\right)=f\left(\tau ; 0, \eta_{0}\right)-\eta_{0} \\
d^{\prime}\left(\eta_{0}\right)=0 \\
d^{\prime \prime}\left(\eta_{0}\right)= \pm 2 \int_{0}^{\tau} a_{1}(s) d s
\end{gathered}
$$

b) Segue de um cálculo direto da divergência do campo

$$
\begin{aligned}
\widetilde{X}_{1}(f)= & \operatorname{sen}(v) \frac{\partial}{\partial u}+\left(a_{0}(u) \cos ^{3}(v)+a_{1}(u) \operatorname{sen}(v) \cos ^{2}(v)\right. \\
& \left.+a_{2}(u) \operatorname{sen}^{2}(v) \cos (v)\right) \frac{\partial}{\partial v}
\end{aligned}
$$

Para $\left.\gamma_{+}=\left(u,+\frac{\pi}{2}\right)\right), \operatorname{div} D \tilde{X}_{1}\left(\gamma_{+}\right)=-a_{2}(u)$.

Para $\left.\gamma_{-}=\left(-u,-\frac{\pi}{2}\right)\right), \operatorname{div} D \tilde{X}_{1}\left(\gamma_{-}\right)=a_{2}(u)$. 


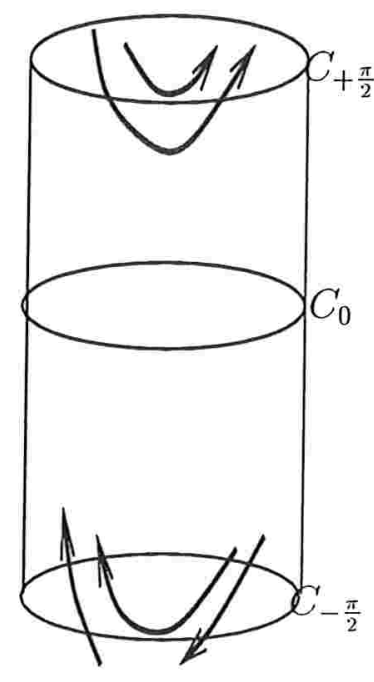

$a_{3}^{\prime}\left(u_{*}\right)>0$

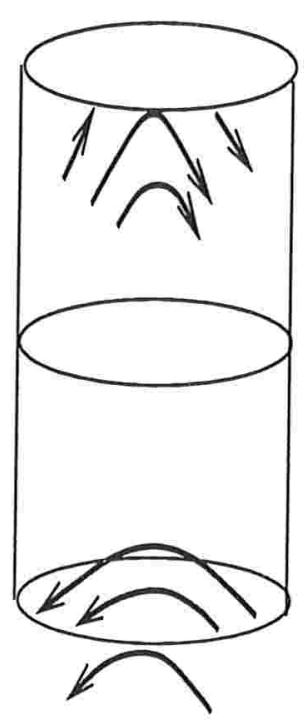

$a_{3}^{\prime}\left(u_{*}\right)<0$

Figura 2.3: Tangências aos círculos $C_{ \pm \frac{\pi}{2}}$ para $n=3$.

Proposição 2.5 Seja $X(f) \in \mathcal{E}^{n, r}(M) \operatorname{com} f(x, y)=\sum_{i=0}^{n} a_{i}(x) y^{i}$ de classe $C^{r}, r \geq 1$ e $n=3$. Então, as trajetórias de $\tilde{X}_{1}(f)$ cruzam transversalmente os círculos $C_{ \pm \frac{\pi}{2}}$, fronteira de $\widetilde{M}$, salvo em pontos isolados da forma $\left(u_{*}, \pm \frac{\pi}{2}\right)$ onde $u_{*}$ é raíz de $a_{3}(u)=0$. Além disso, esses pontos são pontos de tangência parabólica se $a_{3}^{\prime}\left(u_{*}\right) \neq 0$.

Prova. Consideramos a função $b: \widetilde{M} \rightarrow \Re$ definida por $b(u, v)=\cos (v)$ satisfazendo as propriedades $b(u, v)>0$ no $\operatorname{int}(\widetilde{M})$ e $b(u, v)=0$ para $(u, v) \in$ $C_{ \pm \frac{\pi}{2}}$.

A condição de transversalidade é dada por

$$
\widetilde{X}_{1}(f) \cdot b(u, v) \doteq<\widetilde{X}_{1}(f)(u, v), \nabla b(u, v)>\neq 0 .
$$

Com nossos dados, a condição se escreve

$$
\widetilde{X}_{1}(f) \cdot b(u, v)=-\sum_{i=0}^{n} a_{i}(u) \operatorname{sen}^{i+1}(v) \cos ^{n-i}(v)
$$

e para $(u, v) \in C_{ \pm \frac{\pi}{2}}, \widetilde{X}_{1}(f) \cdot b(u, v)=-( \pm 1)^{n+1} a_{n}(u)$.

Segue que em pontos diferentes de $\left(u_{*}, \pm \frac{\pi}{2}\right)$ com $u_{*}$ raíz de $a_{n}(u)=0$, o campo $\widetilde{X}_{1}(f)$ é transversal aos círculos $C_{ \pm \frac{\pi}{2}}$. 
A condição de tangência parabólica é dada pelas relações $\widetilde{X}_{1}(f) \cdot b=0$ e $\tilde{X}_{1}^{2}(f) \cdot b(u, v) \doteq \widetilde{X}_{1}(f) \cdot\left(\widetilde{X}_{1}(f) \cdot b\right)(u, v) \neq 0$.

Nos pontos $\left(u, \pm \frac{\pi}{2}\right)$, essa condição é da forma

$$
\widetilde{X}_{1}^{2}(f) \cdot b\left(u, \pm \frac{\pi}{2}\right)=\mp a_{3}^{\prime}(u) \pm a_{3}(u) a_{2}(u) .
$$

Portanto, temos tangência parabólica se $a_{3}\left(u_{*}\right)=0$ e $a_{3}^{\prime}\left(u_{*}\right) \neq 0$. Mais ainda, a tangência é externa (resp. interna) em $C_{+\frac{\pi}{2}}$ e interna (resp. externa) em $C_{-\frac{\pi}{2}}$ quando $a_{3}^{\prime}\left(u_{*}\right)>0$ (resp. $a_{3}^{\prime}\left(u_{*}\right)<0$ ). Ver figura 2.3.

Proposição 2.6 Dado $X(f) \in \mathcal{E}^{n, r}(M)$, com $f(x, y)=\sum_{i=0}^{n} a_{i}(x) y^{i}$ de classe $C^{r}, r \geq 1$ e $n \geq 4$. Então

a) para $n=4, \widetilde{X}_{1}(f)$ tem singularidades nos círculos $C_{ \pm \frac{\pi}{2}}$ da forma $\left(u_{*}, \pm \frac{\pi}{2}\right)$ com $u_{*}$ raíz de $a_{4}\left(u_{*}\right)=0$. Essas singularidádes são hiperbólicas se $a_{4}^{\prime}\left(u_{*}\right) \neq 0$ e $a_{3}\left(u_{*}\right) \neq 0$, semi-hiperbólicas se $a_{4}^{\prime}\left(u_{*}\right)=0$ e $a_{3}\left(u_{*}\right) \neq 0$, nilpotente se $a_{4}^{\prime}\left(u_{*}\right)=0$ e $a_{3}\left(u_{*}\right)=0$.

b) para $n>4, \widetilde{X}_{1}(f)$ tem singularidades nos círculos $C_{ \pm \frac{\pi}{2}}$ da forma $\left(u_{*}, \pm \frac{\pi}{2}\right)$ com $u_{*}$ raíz de $a_{n}(u)=0$. Essas singularidades são do tipo semi-hiperbólico se $a_{n-1}\left(u_{*}\right) \neq 0$ e nilpotente se $a_{n-1}\left(u_{*}\right)=0 e$ $a_{n}^{\prime}\left(u_{*}\right) \neq 0$.

Prova. a) Para $n=4$, o campo

$$
\widetilde{X}_{1}(f)\left\{\begin{array}{l}
u^{\prime}=\operatorname{sen}(v) \cos (v) \\
u^{\prime}=\sum_{i=0}^{4} a_{i}(u) \operatorname{sen}^{i}(v) \cos ^{4-i}(v)
\end{array}\right.
$$

tem singularidades da forma $\left(u_{*}, \pm \frac{\pi}{2}\right)$ onde $u_{*}$ é um zero de $a_{4}(u)=0$. A matriz jacobiana de $\widetilde{X}_{1}(f)$ avaliada nesses pontos é dada por

$$
D \tilde{X}_{1}(f)\left(u_{*}, \pm \frac{\pi}{2}\right)=\left(\begin{array}{cc}
0 & -1 \\
a_{4}^{\prime}\left(u_{*}\right) & -a_{3}\left(u_{*}\right)
\end{array}\right) .
$$

Logo, com os valores do $\operatorname{det}\left(D \tilde{X}_{1}(f)\right)=a_{4}^{\prime}\left(u_{*}\right)$ e do $\operatorname{traço}\left(D \tilde{X}_{1}(f)\right)=$ $-a_{3}\left(u_{*}\right)$ classificamos as singularidades como segue:

Se $a_{4}^{\prime}\left(u_{*}\right)<0,\left(u_{*}, \pm \frac{\pi}{2}\right)$ é uma sela.

Se $a_{4}^{\prime}\left(u_{*}\right)>0$ e $a_{3}^{2}\left(u_{*}\right)-4 a_{4}^{\prime}\left(u_{*}\right)>0,\left(u_{*}, \pm \frac{\pi}{2}\right)$ é um nó. Notamos que os tipos de nós possíveis são atrator, repulsor e confluente. O nó impróprio não pode acontecer pois as trajetórias sobre os círculos não estão permitidas nestes campos (ver Proposição 2.5). 
Se $a_{4}^{\prime}\left(u_{*}\right)>0$ e $a_{3}^{2}\left(u_{*}\right)-4 a_{4}^{\prime}\left(u_{*}\right)<0,\left(u_{*}, \pm \frac{\pi}{2}\right)$ é um foco.

E por último, se $a_{4}^{\prime}\left(u_{*}\right)>0$ e $a_{3}\left(u_{*}\right)=0,\left(u_{*}, \pm \frac{\pi}{2}\right)$ é um centro.

Para melhor determinar o comportamento topológico dessas singularidades, calculamos os auto-espaços de $D \widetilde{X}(f)$. Os autovalores têm as expressões $\lambda_{ \pm}=-\frac{1}{2}\left(a_{3}\left(u_{*}\right) \pm \sqrt{\left.a_{3}^{2}\left(u_{*}\right)-4 a_{4}^{\prime}\left(u_{*}\right)\right)}\right.$ com vetores próprios associados $v_{ \pm}=\left(1, \lambda_{ \pm}\right)$.

Encontramos singularidades do tipo semi-hiperbólico se $a_{4}^{\prime}\left(u_{*}\right)=0 \mathrm{e}$ $a_{3}\left(u_{*}\right) \neq 0$, e do tipo nilpotente se $a_{4}^{\prime}\left(u_{*}\right)=0$ e $a_{3}\left(u_{*}\right)=0$.

b) Para $n>4$, o campo

$$
\tilde{X}_{1}(f)\left\{\begin{aligned}
u^{\prime} & =\operatorname{sen}(v) \cos ^{n-3}(v) \\
v^{\prime} & =\sum_{i=0}^{n} a_{i}(u) \operatorname{sen}^{i}(v) \cos ^{n-i}(v)
\end{aligned}\right.
$$

tem sua matriz jacobiana avaliada em uma singularidade $\left(u_{*}, \pm \frac{\pi}{2}\right)$ da forma

$$
D \tilde{X}(f)_{1}\left(u_{*}, \pm \frac{\pi}{2}\right)=\left(\begin{array}{cc}
0 & 0 \\
( \pm 1)^{n} a_{n}^{\prime}\left(u_{*}\right) & ( \pm 1)^{n} a_{n-1}\left(u_{*}\right)
\end{array}\right) .
$$

Assim, a singularidade $\left(u_{*}, \pm \frac{\pi}{2}\right)$ é do tipo semi-hiperbólico se $a_{n-1}\left(u_{*}\right) \neq 0 \mathrm{e}$ do tipo nilpotente se $a_{n}^{\prime}\left(u_{*}\right) \neq 0$ e $a_{n-1}\left(u_{*}\right)=0$.

Para determinar o tipo topológico das singularidades semi-hiperbólicas e nilpotentes, usaremos o campo $\widetilde{X}_{2}(f)$. Lembramos que a mudança de variáveis $u=x$ e $v=\frac{1}{y}$ para $y \neq 0$ transforma uma vizinhança aberta $U$ do infinito de $M$ no conjunto semi-aberto $\widetilde{U}$ contendo o eixo $u$. O infinito de $M$ é levado no círculo $\widetilde{C}_{0}$. O campo $X(f)=X\left(\sum_{i=0}^{n} a_{i}(x) y^{i}\right)$ é transformado no campo

$$
\tilde{X}_{2}(f)=v^{n-3} \frac{\partial}{\partial u}-\sum_{i=0}^{n} a_{i}(u) v^{n-i} \frac{\partial}{\partial v}
$$

para $n \geq 4$ multiplicando previamente pela função $v^{n-2}$.

Observação 2.3.1 Quando $n=4$, o campo $\tilde{X}_{2}(f)$ está associado a uma equação diferencial de segunda ordem. Assim, todas as propriedades de $X(f)$ em $C_{0}$ citadas no começo do capítulo são aplicadas a $\widetilde{X}_{2}(f)$ em $C_{ \pm \frac{\pi}{2}}$. Em particular, o campo $\widetilde{X}_{2}(f)$ não tem trajetórias sobre o círculo $\widetilde{C}_{0}=S^{1} \times\{0\}$.

Nas seguintes proposições, apresentamos o tipo topológico de uma singularidade $\left(u_{*}, 0\right)$ semi-hiperbólica, para $n \geq 4$. 
Proposição 2.7 Seja $X(f) \in \mathcal{E}^{n, r} \operatorname{com} f(x, y)=\sum_{i=0}^{n} a_{i}(x) y^{i}$ de classe $C^{r}$, $r \geq 2$ e $n=4$. Seja $\left(u_{*}, 0\right)$ uma singularidade semi-hiperbólica de $\tilde{X}(f)$ e seja $k \in N, k \geq 2$ tal que $a_{4}^{(k)}\left(u_{*}\right) \neq 0$ e $a_{4}^{(j)}\left(u_{*}\right)=0$ para $j<k$. Então, $a$ singularidade $\left(u_{*}, 0\right)$ tem um dos seguintes tipos topológicos

a) Se $k$ é ímpar e $a_{4}^{(k)}\left(u_{*}\right)>0$, então $\left(u_{*}, 0\right)$ é topologicamente equivalente a um nó. Ver figura 2.4 a).

b) Se $k$ é ímpar e $a_{4}^{(k)}\left(u_{*}\right)<0$, então $\left(u_{*}, 0\right)$ é topologicamente equivalente a uma sela. Ver figura 2.4 b).

c) Se $k$ é par, então $\left(u_{*}, 0\right)$ é uma sela-nó. A variedade hiperbólica é tangente à reta $v=-a_{3}\left(u_{*}\right) u$, instável se $a_{3}\left(u_{*}\right)<0$ e estável se $a_{3}\left(u_{*}\right)>0$. A variedade central é tangente ao eixo $u$ e aponta na direção positiva se $a_{4}^{(k)}\left(u_{*}\right) \cdot a_{3}\left(u_{*}\right)<0$, ou na direção negativa se $a_{4}^{(k)}\left(u_{*}\right) \cdot a_{3}\left(u_{*}\right)>0$. Ver figura 2.5.

Prova. Podemos supor $u_{*}=0$. Calculamos a restrição do campo $\widetilde{X}_{2}(f)$ à variedade central $W_{c}$ para o caso $a_{4}^{\prime}\left(u_{*}\right)=0$ e $a_{3}\left(u_{*}\right) \neq 0$.

A variedade central é tangente ao auto-espaço $T_{c}$ associado ao autovalor nulo e gerado pelo vetor $(1,0)$. Podemos representar $W_{c}$ como o gráfico de uma função $h: \Re \rightarrow \Re$ de classe $C^{r}$,

$$
W_{c}=\left\{(u, v) \in \Re^{2}: v=h(u)\right\} .
$$

Pelas condições de tangências de $W_{c}, h\left(u_{*}\right)=h^{\prime}\left(u_{*}\right)=0$.

A restrição do campo à variedade central tem a forma

$$
\left\{\begin{array}{l}
u^{\prime}=h(u) \\
v^{\prime}=-\left(\sum_{i=0}^{4} a_{i}(u) h^{4-i}(u)\right)
\end{array}\right.
$$
função

Substituindo $v=h(u)$ na segunda equação do campo, conseguimos a

$$
\phi(u)=h^{\prime}(u) h(u)+\sum_{i=0}^{4} a_{i}(u) h^{4-i}(u)
$$

A função $\phi$ é nula para todo $u$.

Seja $k \in N, k \geq 2$ tal que $a_{4}^{(k)}\left(u_{*}\right) \neq 0$ e $a_{4}^{(j)}\left(u_{*}\right)=0$ para $j<k$. 
Escrevemos $h(u)=h_{2} u^{2}+\cdots+h_{k} u^{k}+\cdots$. Então, temos

$$
\begin{aligned}
\phi\left(u_{*}\right)= & 0 \\
\phi^{\prime}\left(u_{*}\right)= & 0 \\
\phi^{\prime \prime}\left(u_{*}\right)= & 2 a_{3}\left(u_{*}\right) h_{2}=0 \\
\phi^{\prime \prime \prime}\left(u_{*}\right)= & 3 !\left(2 h_{2}^{2}+a_{3}\left(u_{*}\right) h_{3}\right)=0 \\
\vdots & \\
\phi^{(k)}\left(u_{*}\right)= & k !\left(a_{4}^{(k)}\left(u_{*}+a_{3}\left(u_{*}\right) h_{k}\right)\right. \\
& \left.+a_{3}^{\prime}\left(u_{*}\right) h_{k-1}+\cdots+a_{3}^{(k-2)}\left(u_{*}\right) h_{2}\right) \\
& +A_{3 k}\left(h_{2}, \cdots, h_{k-1}\right)+A_{2 k}\left(h_{2}, \cdots, h_{k-2}\right)+A_{1 k}\left(h_{2}, \cdots, h_{k-3}\right) \\
& +A_{0 k}\left(h_{2}, \ldots, h_{k-4}\right)=0
\end{aligned}
$$

onde $A_{i k}=\frac{d^{k}}{d u^{k}}\left(a_{i} \cdot h^{4-i}\right)\left(u_{*}\right)$ para $i=0, \ldots, 3$.

Resolvemos essas equações com respeito aos coeficientes $h_{i}$, obtendo

$$
h_{2}=h_{3}=\cdots=h_{k-1}=0
$$

e

$$
h_{k}=-\frac{a_{4}^{(k)}}{a_{3}\left(u_{*}\right)}
$$

A função $h(u)$ tem a forma

$$
h(u) \equiv \alpha u^{k}+O\left(u^{k+1}\right)
$$

onde $\alpha=-\frac{a_{4}^{(k)}\left(u_{*}\right)}{a_{3}\left(u_{*}\right)}$.

A restrição do campo à variedade central tem a forma

$$
u^{\prime}=\alpha u^{k}+O\left(u^{k+1}\right)
$$

As conclusões da proposição seguem de analisar o sinal de $\alpha$ e a orientação da variedade hiperbólica. 

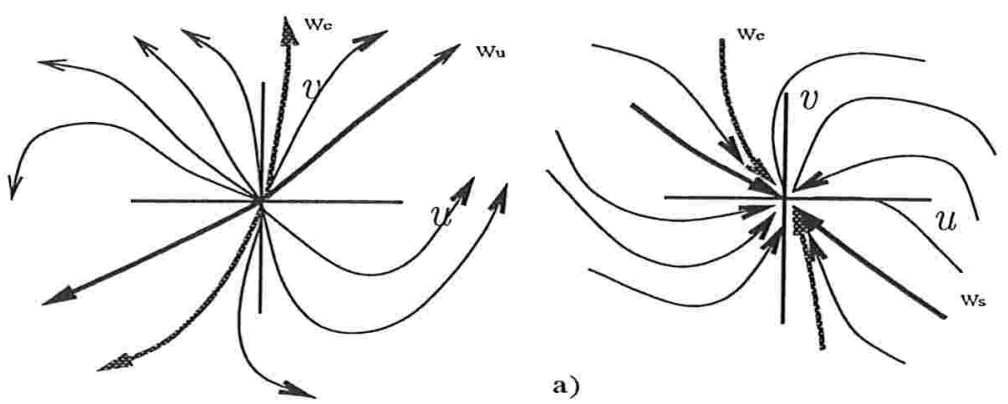

a)
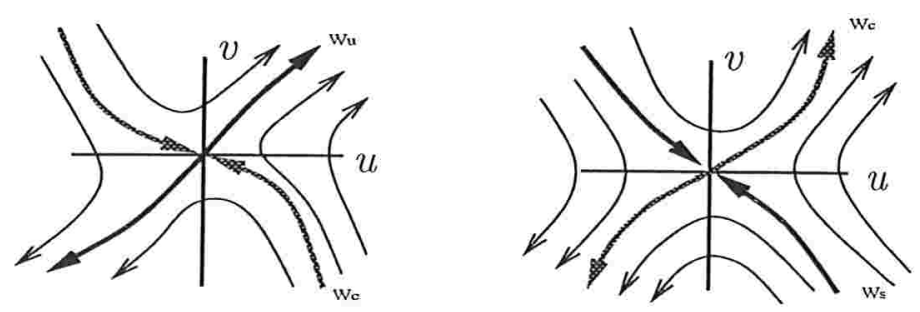

b)

Figura 2.4: Retratos de fase das singularidades semi-hiperbólicas dos tipos a) nó , b) sela. $n=4$

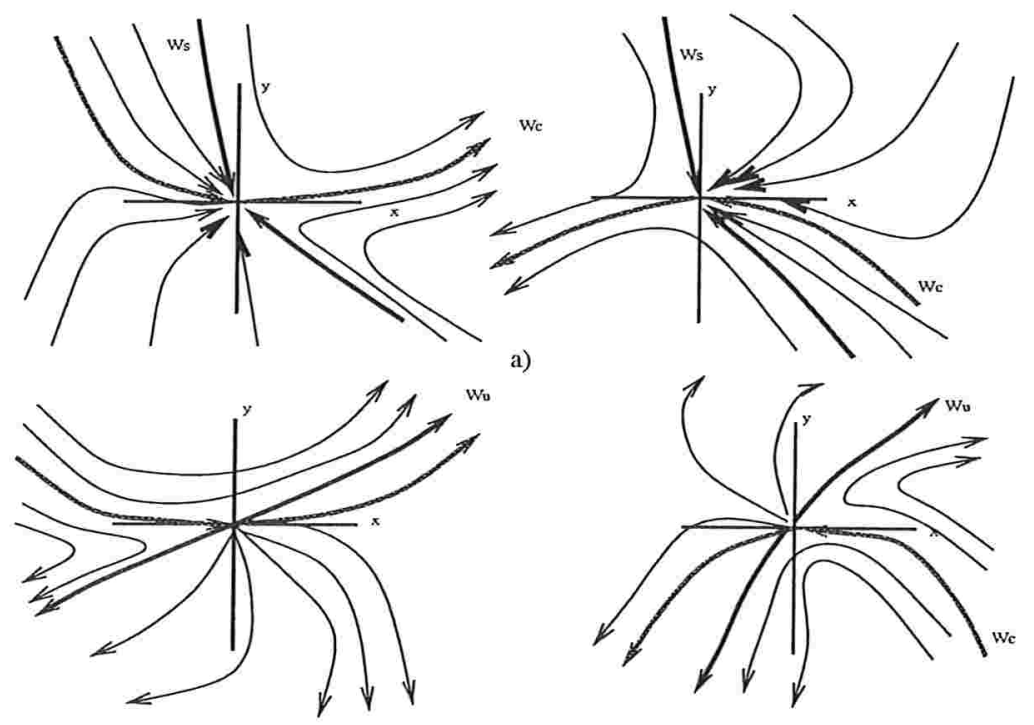

b)

Figura 2.5: Retratos de fase das singularidades semi-hiperbólicas do tipo sela-nó: a) $\left.a_{3}\left(u_{*}\right)>0, b\right) a_{3}\left(u_{*}\right)<0$. 
Proposição 2.8 Seja $X(f) \in \mathcal{E}^{n, r} \operatorname{com} f(x, y)=\sum_{i=0}^{n} a_{i}(x) y^{i}$ de classe $C^{r}$, $r \geq 2$ e $n>4$. Seja $\left(u_{*}, 0\right)$ uma singularidade semi-hiperbólica de $\tilde{X}(f)$ e seja $k \in N, k \geq 1$ tal que $a_{n}^{(k)}\left(u_{*}\right) \neq 0$ e $a_{n}^{(j)}\left(u_{*}\right)=0$ para $j<k$. Então, $a$ singularidade $\left(u_{*}, 0\right)$ tem um dos seguintes tipos topológicos

i) $k \geq 2$

a) Se $n$ é par, $k$ é ímpar, $a_{n}^{(k)}\left(u_{*}\right) \cdot a_{n-1}\left(u_{*}\right)<0$ e $a_{n-1}\left(u_{*}\right)<0$ então $\left(u_{*}, 0\right)$ é topologicamente equivalente a um nó. Ver figura 2.6 a).

b) Se $n$ é par, $k$ é ímpar, $a_{n}^{(k)}\left(u_{*}\right) \cdot a_{n-1}\left(u_{*}\right)<0$ e $a_{n-1}\left(u_{*}\right)>0$ então $\left(u_{*}, 0\right)$ é topologicamente equivalente a uma sela. Ver figura 2.6 b).

c) Se $n$ é par, $k$ é ímpar, $a_{n}^{(k)}\left(u_{*}\right) \cdot a_{n-1}\left(u_{*}\right)>0$ e $a_{n-1}\left(u_{*}\right)<0$ então $\left(u_{*}, 0\right)$ é topologicamente equivalente a uma sela. Ver figura 2.6 c).

d) Se n é par, $k$ é ímpar, $a_{n}^{(k)}\left(u_{*}\right) \cdot a_{n-1}\left(u_{*}\right)>0$ e $a_{n-1}\left(u_{*}\right)>0$ então $\left(u_{*}, 0\right)$ é topologicamente equivalente a um nó. Ver figura $\left.2.6 \mathrm{~d}\right)$.

e) Se $(n-3) \cdot k$ é par então $\left(u_{*}, 0\right)$ é uma sela-nó. A variedade hiperbólica é tangente ao eixo $v$. A variedade central é tangente ao eixo $u$ e aponta na direção positiva se $a_{n}^{(k)}\left(u_{*}\right) \cdot a_{n-1}\left(u_{*}\right)<0$, ou na direção negativa se $a_{n}^{(k)}\left(u_{*}\right) \cdot a_{n-1}\left(u_{*}\right)>0$. Ver figura 2.7 .

ii) $k=1$.

a) Se n é par, $a_{n}^{(k)}\left(u_{*}\right) \cdot a_{n-1}\left(u_{*}\right)<0$ e $a_{n-1}\left(u_{*}\right)<0$ então $\left(u_{*}, 0\right)$ é topologicamente equivalente a um nó. Ver figura 2.6 a).

b) Se $n$ é par, $a_{n}^{(k)}\left(u_{*}\right) \cdot a_{n-1}\left(u_{*}\right)<0$ e $a_{n-1}\left(u_{*}\right)>0$ então $\left(u_{*}, 0\right)$ é topologicamente equivalente a uma sela. Ver figura 2.6 b).

c) Se n é par, $a_{n}^{(k)}\left(u_{*}\right) \cdot a_{n-1}\left(u_{*}\right)>0$ e $a_{n-1}\left(u_{*}\right)<0$ então $\left(u_{*}, 0\right)$ é topologicamente equivalente a uma sela. Ver figura $2.6 \mathrm{c}$ ).

d) Se $n$ é par, $a_{n}^{(k)}\left(u_{*}\right) \cdot a_{n-1}\left(u_{*}\right)>0$ e $a_{n-1}\left(u_{*}\right)>0$ então $\left(u_{*}, 0\right)$ é topologicamente equivalente a um nó. Ver figura 2.6 d).

e) Se $n$ é ímpar então $\left(u_{*}, 0\right)$ é uma sela-nó. A variedade hiperbólica é tangente ao eixo $v$. A variedade central é tangente à reta $v=$ $-\frac{a_{n}^{(k)}\left(u_{*}\right)}{a_{n-1}\left(u_{*}\right)} u$ e aponta na direção positiva se $a_{n}^{(k)}\left(u_{*}\right) \cdot a_{n-1}\left(u_{*}\right)<0$, ou na direção negativa se $a_{n}^{(k)}\left(u_{*}\right) \cdot a_{n-1}\left(u_{*}\right)>0$. Ver figura 2.8. 


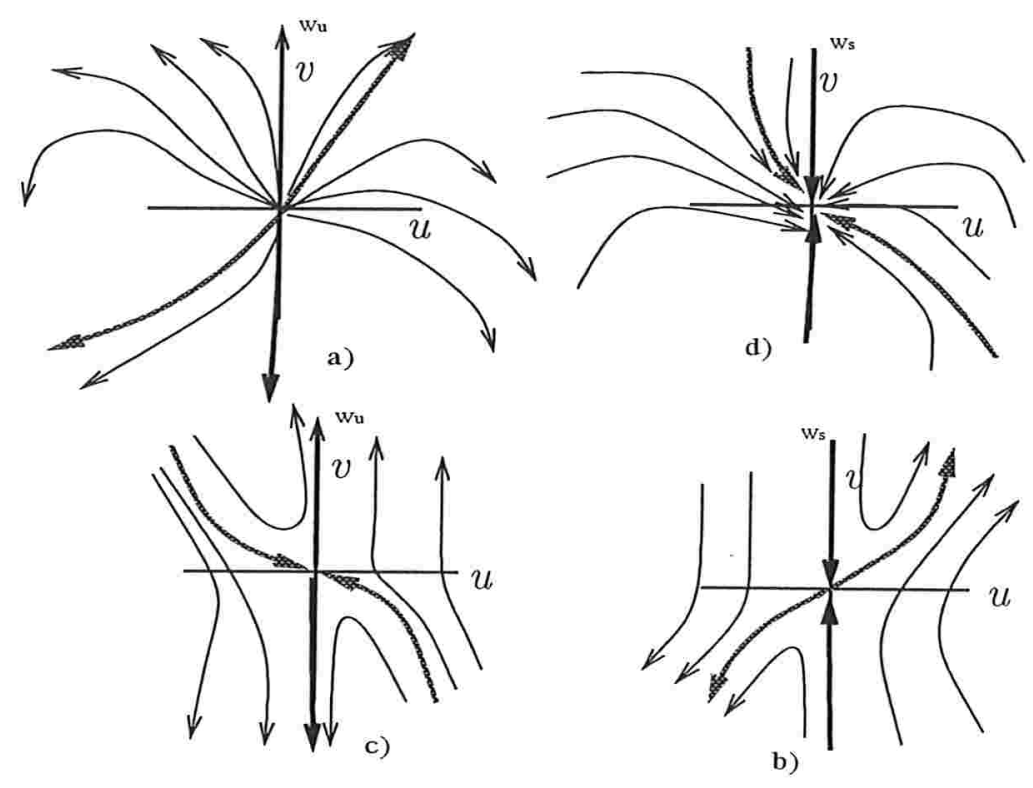

Figura 2.6: Retratos de fase das singularidades semi-hiperbólicas dos tipos nó e sela. $n \geq 5$

Prova. O auto-espaço $E_{0}$ associado ao autovalor nulo é gerado pelo vetor

i) $V_{0}=(1,0)$, se $a_{n}^{\prime}\left(u_{*}\right)=0$.

ii) $V_{0}=\left(1,-\frac{a_{n}^{\prime}\left(u_{*}\right)}{a_{n-1}\left(u_{*}\right)}\right)$, se $a_{n}^{\prime}\left(u_{*}\right) \neq 0$.

$\mathrm{O}$ auto-espaço $E_{1}$ associado ao autovalor não nulo é gerado pelo vetor $V_{1}=(0,1)$.

A variedade central $W_{c}$ tem que ser tangente à reta que passa pela origem na direção $V_{0}$. Podemos representar $W_{c}$ como o gráfico de uma função $h$ : $\Re \rightarrow \Re$ de classe $C^{r}$

$$
W_{c}=\left\{(u, v) \Re^{2}: v=h(u)\right\}
$$

De fato, a função $Q(u, v)=\sum_{i=0}^{n} a_{i}(u) v^{n-i}$ tem as condições $Q\left(u_{*}, 0\right)=0$, e $\frac{\partial Q}{\partial v}\left(u_{*}, 0\right)=-a_{n-1}\left(u_{*}\right) \neq 0$. Pelo Teorema da Função Implícita existe uma função $h: \mathcal{U} \rightarrow \Re$ de classe $C^{r}$ definida numa vizinhança $\mathcal{U}$ de $u_{*}$ tal que $Q(u, h(u))=0$ para todo $u \in \mathcal{U}$.

Segue que a restrição do campo $\widetilde{X}(f)$ à variedade central é descrita pela função

$$
g(u)=\widetilde{X}(f)_{\mid W_{c}}=h^{n-3}(u) .
$$

Precisamos, então, descrever o comportamento assintótico da $g(u)$ em 




b)

Figura 2.7: Retratos de fase das singularidades semi-hiperbólicas do tipo sela-nó. $n \geq 5$ e $k \geq 2$
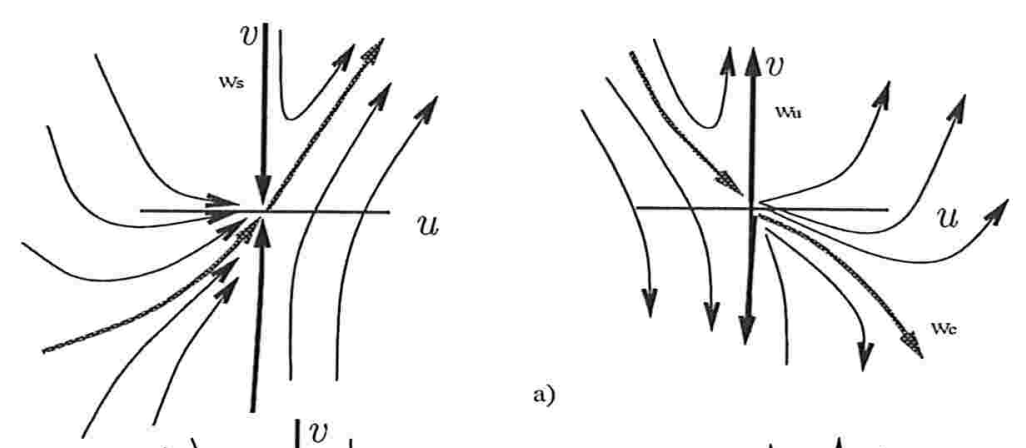

a)
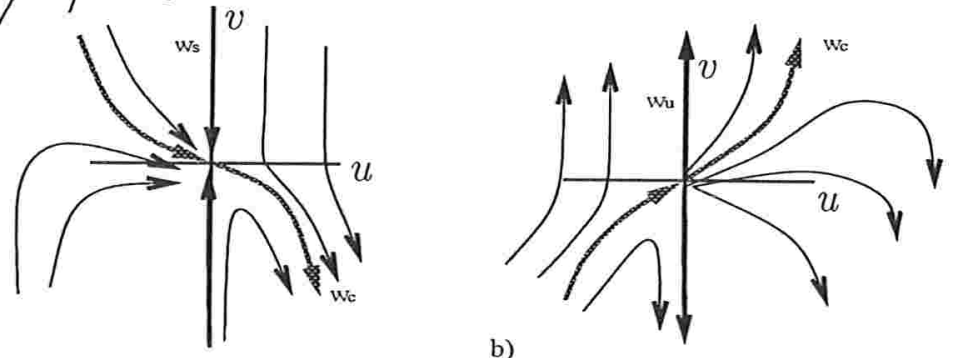

Figura 2.8: Retratos de fase das singularidades semi-hiperbólicas do tipo sela-nó. $n \geq 5$ e $k=1$ 
uma vizinhança de $u_{*}$. Para isso, consideramos a função $\varphi$ definida por

$$
\varphi(u)=h^{\prime}(u) h^{n-3}(u)+\sum_{i=0}^{n} a_{i}(u) h^{n-i}(u) .
$$

Essa função é identicamente nula, e é obtida substituindo $v=h(u)$ na equação $v^{\prime}=Q(u, v)$.

Seja $k \in N, k \geq 1$ tal que $a_{n}^{(k)}\left(u_{*}\right) \neq 0$ e $a_{n}^{(j)}\left(u_{*}\right)=0$ para $j<k$.

Para facilitar os cálculos, suporemos $u_{*}=0$

Derivando $\varphi$ várias vezes, podemos obter as derivadas de $h$ em $u_{*}$.

Caso i). $k \geq 2$. Pelas condições de tangências de $h(u)$, podemos escrever $h(u)=h_{2} u^{2}+\cdots+h_{k} u^{k}+\cdots$. Então,

$$
\begin{aligned}
\phi\left(u_{*}\right)= & 0 \\
\phi^{\prime}\left(u_{*}\right)= & 0 \\
\phi^{\prime \prime}\left(u_{*}\right)= & 2 a_{n-1}\left(u_{*}\right) h_{2}=0 \\
\phi^{\prime \prime \prime}\left(u_{*}\right)= & 3 !\left(2 h_{2}^{2}+a_{n-1}\left(u_{*}\right) h_{3}\right)=0 \\
\vdots & \\
\phi^{(k)}\left(u_{*}\right)= & k !\left(a_{n}^{(k)}\left(u_{*}\right)+a_{n-1}\left(u_{*}\right) h_{k}\right) \\
& \left.+a_{n-1}^{\prime}\left(u_{*}\right) h_{k-1}+\cdots+a_{n-1}^{(k-2)}\left(u_{*}\right) h_{2}\right) \\
& +A_{3 k}\left(h_{2}, \cdots, h_{k-1}\right)+A_{2 k}\left(h_{2}, \cdots, h_{k-2}\right)+A_{1 k}\left(h_{2}, \cdots, h_{k-3}\right) \\
& +A_{0 k}\left(h_{2}, \ldots, h_{k-4}\right)=0 \\
&
\end{aligned}
$$

onde $A_{i k}=\frac{d^{k}}{d u^{k}}\left(a_{i} \cdot h^{4-i}\right)\left(u_{*}\right)$ para $i=0, \ldots, n-1$.

Resolvemos as equações (2.9) para os coeficientes $h_{i}$, obtendo

$$
h_{2}=h_{3}=\cdots=h_{k-1}=0
$$

$\mathrm{e}$

$$
h_{k}=-\frac{a_{n}^{(k)}}{a_{n-1}\left(u_{*}\right)}
$$

A função $h(u)$ tem a forma

$$
h(u) \equiv \alpha u^{k}+O\left(u^{k+1}\right)
$$

onde $\alpha=-\frac{a_{n}^{(k)}\left(u_{*}\right)}{a_{n-1}\left(u_{*}\right)}$.

A restriçã̃o do campo à variedade central tem a forma

$$
g(u)=\alpha^{n-3} u^{k(n-3)}+O\left(u^{k+1(n-2)}\right) .
$$

A conclusão do caso $i$ ) segue de observar a paridade de $n$ e de $k$, e os sinais de $a_{n}^{(k)}$ e $a_{n-1}$. 
Caso ii). Com as condições $h\left(u_{*}\right)=0, a_{n}\left(u_{*}\right)=0, a_{n}^{\prime}\left(u_{*}\right) \neq 0 \mathrm{e}$ $a_{n-1}\left(u_{*}\right) \neq 0$, as primeiras duas derivadas de $\varphi$ avaliadas em $u=u_{*}$ têm as expressões

$$
\begin{gathered}
\varphi^{\prime}\left(u_{*}\right)=a_{n}^{\prime}\left(u_{*}\right)+a_{n-1}\left(u_{*}\right) h^{\prime}\left(u_{*}\right)=0 \\
\varphi^{\prime \prime}\left(u_{*}\right)=\begin{array}{l}
(n-3)(n-4) h^{n-5}\left(u_{*}\right)\left(h^{\prime}\left(u_{*}\right)^{3}+a_{n}^{\prime \prime}\left(u_{*}\right)+2 a_{n-1}^{\prime}\left(u_{*}\right) h^{\prime}\left(u_{*}\right)\right. \\
+2 a_{n-2}\left(u_{*}\right)\left(h^{\prime}\left(u_{*}\right)\right)^{2}+a_{n-1}\left(u_{*}\right) h^{\prime \prime}\left(u_{*}\right)=0
\end{array}
\end{gathered}
$$

Resolvendo essas equações para $h\left(u_{*}\right)$ e suas derivadas, obtemos

$$
\begin{gathered}
h^{\prime}\left(u_{*}\right)=-\frac{a_{n}^{\prime}\left(u_{*}\right)}{a_{n-1}\left(u_{*}\right)} \\
h^{\prime \prime}\left(u_{*}\right)=-\frac{a_{n}^{\prime \prime}\left(u_{*}\right)}{a_{n-1}\left(u_{*}\right)}+2 \frac{a_{n-1}^{\prime}\left(u_{*}\right)}{a_{n-1}\left(u_{*}\right)} \frac{a_{n}^{\prime}\left(u_{*}\right)}{a_{n-1}\left(u_{*}\right)}-2 \frac{a_{n-2}\left(u_{*}\right)}{a_{n-1}\left(u_{*}\right)}\left(\frac{a_{n}^{\prime}\left(u_{*}\right)}{a_{n-1}\left(u_{*}\right)}\right)^{2}
\end{gathered}
$$
te.

Devemos somar a $h^{\prime \prime}\left(u_{*}\right)$ o termo $\frac{2}{a_{n-1}\left(u_{*}\right)}\left(\frac{a_{n}^{\prime}}{a_{n-1}\left(u_{*}\right)}\right)^{3}$ quando $n=5$ somen-

Logo, a restrição do campo $\widetilde{X}(f)$ à variedade central dada pela função $g(u)$ tem o comportamento assintótico numa vizinhança de $u_{*}$ da forma

$$
g(u)=A^{n-3}\left(u-u_{*}\right)^{n-3}+O\left(\left(u-u_{*}\right)^{n-2}\right)
$$

onde $A=-\frac{a_{n}^{\prime}\left(u_{*}\right)}{a_{n-1}\left(u_{*}\right)}$.

A conclusão do caso $i$ ) segue de observar a paridade de $n$ e os sinais de $a_{n}^{(k)}$ e $a_{n-1}$. Por exemplo.

Se $n$ é par, $a_{n}^{(k)}<0$ e $a_{n-1}\left(u_{*}\right)>0,\left(u_{*}, 0\right)$ é uma singularidade do tipo sela.

Se $n$ é par, $a_{n}^{(k)}>0$ e $a_{n-1}\left(u_{*}\right)<0,\left(u_{*}, 0\right)$ é uma singularidade do tipo nó.

Se $n$ é ímpar, $\left(u_{*}, 0\right)$ é uma singularidade do tipo sela-nó.

Para mais referências sobre os tipos topológicos das singularidades semihiperbólicas ver [1] pág. 222-225.

Observação 2.3.2 Quando $n=4$, o campo $\widetilde{X}_{2}(f)$ deriva de uma equação de segunda ordem e tem, em uma singularidade nilpotente, a parte linear igual a $y \frac{\partial}{\partial x}$. Quando $n>4$, o campo $\tilde{X}_{2}(f)$ não deriva de uma equação de segunda ordem, mas tem, em uma singularidade nilpotente, a parte linear igual a $x \frac{\partial}{\partial y}$. Assim, a variedade central, no primeiro caso, é tangente ao eixo $x$, no entanto, no segundo caso é tangente ao eixo $y$. 
Uma classificação das singularidades nilpotentes dos campos derivados das equações diferenciais de segunda ordem foi dada em [5], considerando-se a equivalência topológica entre os campos e suas partes principais. Essencialmente, obteve-se oito classes de equivalências topológicas. Essas classes foram encontradas, também, em [3] usando as formas normais do campo. 


\section{Capítulo 3}

\section{Estabilidade estrutural de $X(f)$}

Neste capítulo, definimos para cada $n$ o conjunto $\Sigma^{n} \subset \mathcal{E}^{n, r}$ de campos $X(f)=X\left(\sum_{i=0}^{n} a_{i}(x) y^{i}\right)$ definidos em $M=S^{1} \times \Re$ com propriedades simples que caracterizam os campos vetoriais estruturalmente estáveis.

Denotamos por $\widetilde{X}(f)$ e $\widetilde{M}=S^{1} \times\left[-\frac{\pi}{2}, \frac{\pi}{2}\right]$ a compactificação de $X(f)$ e $M$ respectivamente como foi introduzida nas seções 1.3 e 2.2. Lembramos que $C_{0}=S^{1} \times\{0\}$ e $C_{ \pm \frac{\pi}{2}}=S^{1} \times\left\{ \pm \frac{\pi}{2}\right\}$.

Distinguimos os conjuntos $\Sigma^{n}$ para $n=1, \cdots, 4$ e $n \geq 5$, por causa do comportamento particular do campo $\widetilde{X}(f)$ nos círculos $C_{ \pm \frac{\pi}{2}}$, como especificamos a seguir:

$n=1,2$. Os círculos $C_{ \pm \frac{\pi}{2}}$ constituem órbitas periódicas de $\widetilde{X}(f)$.

$n=3$. O campo $\tilde{X}(f)$ tem trajetórias tangentes aos círculos $C_{ \pm \frac{\pi}{2}}$.

$n=4$. O campo $\widetilde{X}(f)$ tem singularidades hiperbólicas em $C_{ \pm \frac{\pi}{2}}$.

$n \geq 5$. O campo $\widetilde{X}(f)$ tem singularidades semi-hiperbólicas em $C_{ \pm \frac{\pi}{2}}$.

Cada um desses casos será tratado em uma seção deste capítulo, na qual definiremos $\Sigma^{n}$ e provaremos a genericidade de $\Sigma^{n}$ (i,e. $\Sigma^{n}$ é aberto e denso em $\left.\mathcal{E}^{n, r}\right)$. Mostraremos que o conjunto de parâmetros $\mu=\left(\mu_{0}, \ldots, \mu_{k}\right)$ em $\Re^{k+1}$ tais que $X_{\mu}(f)=X\left(f+\mu_{0}+\mu_{1} y+\mu_{k-1} y^{n-1}+\mu_{k} y^{n}\right)$ não pertence a $\Sigma^{n}$, tem medida de Lebesgue zero em $\Re^{k+1}$. O número de parâmetros $k$ depende de $n$ e do comportamento do campo nas vizinhanças dos círculos $C_{0}$ e $C_{ \pm \frac{\pi}{2}}$.

Na seção 3.6, provamos a caracterização de $X(f) \in \mathcal{E}^{n, r}$ estruturalmente estável.

Finalmente, na seção 3.7, apresentamos exemplos de campos $X(f) \in \Sigma^{n}$ que podem formar algumas das regiões canônicas estudadas. 
Em todo o capítulo usaremos a seguinte notação: $\widetilde{X}_{\mu}(f)$ denotará a compactificação de $X_{\mu}(f)$,

$\Delta X(f)(x, y)$, denotará o determinante de $D X(f)(x, y)$, e $\sigma X(f)(x, y)$, denotará o traço de $D X(f)(x, y)$.

\subsection{Caso $n=1$.}

\subsubsection{Definição de $\Sigma^{1}$}

Lembramos que o campo $X(f)$ pode apresentar na parte finita de $M$ órbitas periódicas de tipo homotópica a zero e as que circundam o cilindro sem cortar o círculo $C_{0}$.

Vimos na proposição 2.4 que o campo $\tilde{X}(f)$ tem órbitas periódicas nos círculos $C_{ \pm \frac{\pi}{2}}$. As órbitas periódicas $\gamma_{ \pm}(u)=\left(\phi(u), \pm \frac{\pi}{2}\right)$ com período $\tau$ têm índice de estabilidade $\chi=0$ (i.e. a primeira derivada da aplicação de Poincaré igual a 1).

Definição 3.1 Definimos por $\Sigma^{1}$, o conjunto de campos $X(f)$ de classe $C^{r}$ com $r \geq 2$ tais que suas compactificaçôes $\tilde{X}(f)$ têm :

1. as singularidades hiperbólicas, contidas no círculo $C_{0}$;

2. as órbitas periódicas hiperbólicas, contidas no int $(\widetilde{M})$, e as órbitas periódicas em $C_{ \pm \frac{\pi}{2}}$ semi-estáveis;

3. nenhuma conexão de selas.

Observação 3.1.1 Os campos $X(f) \in \mathcal{E}^{1, r}$ não podem ter trajetórias que escapam ao infinito isoladamente. Pois se existirem, elas devem atingir uma singularidade ou um ponto de tangência no infinito. Ver seção 1.4.

\subsubsection{Genericidade de $\Sigma^{1}$}

Seja $\mu=\left(\mu_{0}, \mu_{1}\right) \in \Re^{2}$.

Teorema 1 Dado $X(f) \in \mathcal{E}^{1, r}(M), r \geq 1$. Então, o conjunto

$$
B^{1}(X(f))=\left\{\mu \in \Re^{2}: X\left(f+\mu_{0}+\mu_{1} y\right) \notin \Sigma^{1}(M)\right\}
$$

é um subconjunto de medida de Lebesgue zero em $\Re^{2}$.

A prova do Teorema 1 segue de vários lemas. 
Lema 3.1 Para $X(f)=X\left(a_{0}(x)+a_{1}(x) y\right) \in \mathcal{E}^{1, r}(M)$ com $r \geq 2$, o conjunto

$$
\begin{gathered}
B_{1}^{1}(X(f))=\left\{\mu_{0} \in \Re: \widetilde{X}\left(f+\mu_{0}\right)\right. \text { tem alguma singularidade } \\
\left.\left(u_{*}, 0\right) \in C_{0} \text { com } \Delta \widetilde{X}\left(f+\mu_{0}\right)=0\right\}
\end{gathered}
$$

tem medida de Lebesgue zero em $\Re$.

Prova. O conjunto de valores críticos de $-a_{0}$ é dado por

$$
\operatorname{Crit}\left(-a_{0}\right)=\left\{\mu_{0} \in \Re: \exists x_{\mu_{0}} \text { tal que }-a_{0}\left(x_{\mu_{0}}\right)=\mu_{0} \text { e } a_{0}^{\prime}\left(x_{\mu_{0}}\right)=0\right\} .
$$

Pelo Lema 1.1 (de Sard), Crit $\left(-a_{0}\right)$ têm medida de Lebesgue zero em $\Re$.

Por outro lado, podemos escrever

$$
\begin{aligned}
\operatorname{Crit}\left(-a_{0}\right)= & \left\{\mu_{0} \in \Re: \exists\left(x_{\mu_{0}}, 0\right) \in C_{0} \text { tal que } \widetilde{X}\left(f+\mu_{0}\right)\left(x_{\mu_{0}}, 0\right)=(0,0)\right. \\
& \left.\operatorname{com} \Delta \tilde{X}\left(f+\mu_{0}\right)\left(x_{\mu_{0}}, 0\right)=0\right\} .
\end{aligned}
$$

Segue que $B_{1}^{1}(X(f))=\operatorname{Crit}\left(-a_{0}\right)$ e portanto tem medida de Lebesgue zero em $\Re$.

Lema $3.2 \operatorname{Dados} X(f)=X\left(a_{0}(x)+a_{1}(x) y\right) \in \mathcal{E}^{1, r}(M), r \geq 1$ e $\mu_{0} \notin$ $B_{1}^{1}(X(f))$. Então, o conjunto

$$
\begin{aligned}
B_{2}^{1}\left(X(f) ; \mu_{0}\right)= & \left\{\mu_{1} \in \Re: \tilde{X}\left(f+\mu_{0}+\mu_{1} y\right)\right. \text { tem alguma } \\
& \text { singularidade não-hiperbólica }\}
\end{aligned}
$$

tem medida de Lebesgue zero em $\Re$.

Prova. Para $\mu_{0} \notin B_{1}^{1}(X(f))$, toda singularidade de $\tilde{X}\left(f+\mu_{0}\right)$, da forma $\left(u_{*}, 0\right) \in C_{0}$, satisfaz $\Delta \widetilde{X}\left(f+\mu_{0}\right)\left(u_{*}, 0\right) \neq 0$.

Seja $k_{\mu_{0}}$ o número de singularidades de $\widetilde{X}\left(f+\mu_{0}+\mu_{1} y\right)$ em $C_{0}$.

Para cada singularidade de $\tilde{X}\left(f+\mu_{0}+\mu_{1} y\right),\left(u_{*, i}, 0\right) \in C_{0}$, existe um único valor $\mu_{1, i}^{*} \in \Re$ tal que $\sigma \widetilde{X}\left(f+\mu_{0}+\mu_{1, i}^{*} y\right)=0$. Pois $\sigma \widetilde{X}\left(f+\mu_{0}+\right.$ $\left.\mu_{1} y\right)=a_{1}(u)+\mu_{1}$. Portanto, $\left(u_{*, i}, 0\right)$ é uma singularidade não-hiperbólica de $\tilde{X}\left(f+\mu_{0}+\mu_{1, i}^{*} y\right)$.

Então, o conjunto $B_{2}^{1}\left(X(f) ; \mu_{0}\right)$ pode ser descrito da seguinte forma

$$
B_{2}^{1}\left(X(f) ; \mu_{0}\right)=\left\{\mu_{1} \in \Re: a_{1}(x)+\mu_{1}=0, a_{0}(x)+\mu_{0}=0 \text { e } a_{0}^{\prime}(x)>0\right\}
$$

Segue que $B_{2}^{1}\left(X(f) ; \mu_{0}\right)$ é um conjunto finito e portanto tem medida zero em $\Re$. 
Lema 3.3 Dado $X(f) \in \mathcal{E}^{1, r}(M)$. Então,

i) Para $\mu_{0} \in \Re$ e $r \geq 1$, o conjunto

$$
\begin{aligned}
B_{3}^{1}\left(X(f) ; \mu_{0}\right)=\left\{\mu_{1} \in \Re:\right. & \tilde{X}\left(f+\mu_{0}+\mu_{1} y\right) \text { tem alguma órbita } \\
& \text { periódica nấ-hiperbólica contida em } M\}
\end{aligned}
$$

tem medida de Lebesgue zero em $\Re$.

ii) Para $r \geq 2$, o conjunto

$$
\begin{aligned}
B_{4}^{1}(X(f))=\left\{\mu_{1} \in \Re:\right. & \tilde{X}\left(f+\mu_{1} y\right) \text { tem alguma órbita } \\
& \text { periódica não semi-hiperbólica em } \left.C_{ \pm \frac{\pi}{2}}\right\}
\end{aligned}
$$

tem medida de Lebesgue zero em $\Re$. tipos:

Prova. $i)$ Uma órbita periódica $\gamma$ de $\widetilde{X}\left(f+\mu_{0}+\mu_{1} y\right)$ é uma dos seguintes

a) homotópica a zero. Contém em seu interior uma singularidade do campo $C_{0}$ e corta transversalmente o eixo $x$.

b) não-homotópica a zero. Circunda o cilindro sem interceptar o eixo $x$.

Para cada situação, encontramos expressões das derivadas da aplicação do primeiro retorno com respeito a um parâmetro. Salientamos que essas expressões serão obtidas para um campo geral $X(f)$ onde $f(x, y)=$ $\sum_{i=0}^{n} a_{i}(x) y^{i}$ e $n \geq 1$.

Começamos com as órbitas periódicas homotópicas a zero.

Caso $a$ ). Para um valor de $\mu_{0} \in \Re$, consideramos uma órbita periódica de $\widetilde{X}\left(f+\mu_{0}+\mu_{1} y\right), \gamma\left(t, p, \mu_{1}\right)=\left(\varphi\left(t, p, \mu_{1}\right), \psi\left(t, p, \mu_{1}\right)\right)$ que passa por $p=\left(x_{0}, 0\right)$ com período $\tau=\tau\left(x_{0}, \mu_{1}\right)$.

Seja $\pi\left(x, \mu_{1}\right)$ a transformação de Poincaré definida em um intervalo $I \subset$ $C_{0}$ com $x_{0} \in I$ e associada ao fluxo do campo $X\left(f+\mu_{0}+\mu_{1} y\right)$.

As derivadas de $\pi\left(x, \mu_{1}\right)$ com respeito a $x$ e $\mu_{1}$ foram calculadas em [1]. Aqui, nós as escrevemos em termos dos coeficientes de $f(x, y)+\mu_{0}+\mu_{1} y$ como segue

$$
\begin{aligned}
\frac{\partial \pi}{\partial x}\left(x_{0}, \mu_{1}\right)= & \prod_{i=1}^{n} \exp \left(\int_{0}^{\tau\left(x_{0}, \mu_{1}\right)}\left(i a_{i}(\varphi(s)) \psi^{i-1}(s)+\mu_{1}\right) d s\right) \\
\frac{\partial \pi}{\partial \mu_{1}}\left(x_{0}, \mu_{1}\right)= & \frac{\frac{\partial \pi}{\partial x}\left(x_{0}, \mu_{1}\right)}{-\left|\gamma^{\prime}\left(0, x_{0}, \mu_{1}\right)\right|} \int_{0}^{\tau\left(x_{0}, \mu_{1}\right)} \prod_{i=1}^{n} \exp \left(-\int_{0}^{s}\left(i a_{i}(\varphi(t)) \psi^{i-1}(t)\right.\right. \\
& \left.\left.+\mu_{1}\right) d t\right) \psi^{2}(s) d s
\end{aligned}
$$


Como $\frac{\partial \pi}{\partial \mu_{1}}\left(x_{0}, \mu_{1}\right) \neq 0$, o Teorema da Função Implícita implica que existe uma única função $\mu_{1}(x)$ definida em uma vizinhança $I_{x_{0}}$ de $x_{0}$ tal que $\pi\left(x, \mu_{1}(x)\right)-x=0$ vale para $\forall x \in I_{x_{0}}$. Derivando a última equação com respeito a $x$, temos

$$
\frac{\partial \pi}{\partial x}\left(x_{0}, \mu_{1}\right)+\frac{\partial \pi}{\partial \mu_{1}}\left(x_{0}, \mu_{1}\right) \frac{\partial \mu_{1}}{\partial x}-1=0
$$

e pelo fato que $\frac{\partial \pi}{\partial \mu_{1}}\left(x_{0}, \mu_{1}\right) \neq 0$, obtemos a condição:

$$
\gamma \text { é não-hiperbólica se e só se } \frac{\partial \mu_{1}}{\partial x}=0 .
$$

Destacamos que para cada $x \in I_{x_{0}}, \mu_{1}(x)$ determina um valor do parâmetro tal que $X\left(f+\mu_{0}+\mu_{1}(x) y\right)$ tem uma órbita periódica passando por $(x, 0)$. Essa órbita periódica é não-hiperbólica quando $\mu_{1}(x)$ for um valor crítico de $\mu_{1}(x): I_{x_{0}} \rightarrow \Re$.

Assim, para cada $\mu_{0} \in \Re$ fixo, o conjunto de valores críticos de $\mu_{1}(x)$ é escrito na forma

$$
\begin{aligned}
O_{1}= & \left\{\mu_{1}(x) \in \Re: X\left(f+\mu_{0}+\mu_{1}(x) y\right)\right. \text { tem uma órbita periódica } \\
& \text { não-hiperbólica homotópica a zero passando por }(x, 0)\} .
\end{aligned}
$$

Caso $b)$. Seja $\gamma(t)=(\varphi(t), \psi(t))$ uma órbita periódica não-homotópica a zero que passa por $\left(0, y_{0}\right)$ com $y_{0} \neq 0$.

A transformação de Poincaré, $\pi\left(y, \mu_{1}\right)$, é definida por

$$
\pi\left(y, \mu_{1}\right)=\Psi\left(\tau, y, \mu_{1}\right)
$$

onde $\tau$ é o período das funções $a_{i}(x)$ e $\Psi\left(x, y, \mu_{1}\right)$ é solução da equação diferencial

$$
\frac{d \Psi}{d x}\left(x, y, \mu_{1}\right)=\frac{1}{\Psi}\left(\sum_{i=0}^{n} a_{i}(x) \Psi^{i}+\mu_{1} \Psi\right)=F\left(x, y, \mu_{1}\right)
$$

com condição inicial $\Psi\left(0, y, \mu_{1}\right)=y$.

A derivada de $\Psi\left(x, y, \mu_{1}\right)$ com respeito ao parâmetro $\mu_{1}$ é justamente a solução da equação linear

$$
\frac{d}{d x}\left(\frac{\partial \Psi}{\partial \mu_{1}}\right)=D_{2} F\left(x, \Psi\left(x, y, \mu_{1}\right), \mu_{1}\right) \frac{\partial \Psi}{\partial \mu_{1}}\left(x, y, \mu_{1}\right)+D_{3} F\left(x, \Psi\left(x, y, \mu_{1}\right), \mu_{1}\right)
$$

onde $D_{2} F\left(x, y, \mu_{1}\right)=-\frac{a_{0}(x)}{y^{2}}$ e $D_{3} F\left(x, y, \mu_{1}\right)=1$. 
Pela equação (3.1), a derivada de $\pi\left(y, \mu_{1}\right)$ com respeito ao parâmetro $\mu_{1}$ é dado por

$$
\frac{\partial \pi}{\partial \mu_{1}}\left(y, \mu_{1}\right)=\exp \left(\mathcal{I}\left(\tau, y, \mu_{1}\right)\right) \int_{0}^{\tau} \exp \left(-\mathcal{I}\left(s, y, \mu_{1}\right)\right) d s
$$

onde $\mathcal{I}\left(t, y, \mu_{1}\right)=\int_{0}^{t} D_{2} F\left(s, \Psi\left(s, y, \mu_{1}\right), \mu_{1}\right) d s$

Da mesma maneira que no caso das órbitas homotópicas a zero, encontramos uma função $\mu_{1}: I_{y_{0}} \rightarrow \Re$ definida em um intervalo do eixo $y$ contendo $y_{0}$ e sem interseção com o eixo $x$ tal que $\pi\left(y, \mu_{1}(y)\right)-y=0$. Derivando essa equação com respeito a $y$, concluímos que

$$
\gamma \text { é não-hiperbólica se e só se } \frac{\partial \mu_{1}}{\partial y}=0 .
$$

Portanto, o conjunto de valores críticos de $\mu_{1}(y)$ é escrito da forma

$$
\begin{aligned}
O_{2}= & \left\{\mu_{1}(\xi) \in \Re: X\left(f+\mu_{0}+\mu_{1}(\xi) y\right)\right. \text { tem uma órbita periódica } \\
& \text { não-hiperbólica circundando o cilindro que passa por }(0, \xi)\}
\end{aligned}
$$

Terminamos a demonstração do lema observando que

$$
B_{3}^{1}\left(X(f) ; \mu_{0}\right)=O_{1} \cup O_{2}
$$

e aplicando o Lema 1.1 (de Sard) aos conjuntos $O_{1}$ e $O_{2}$. Portanto, $B_{3}^{1}\left(X(f) ; \mu_{0}\right)$ tem medida zero em $\Re$.

ii) Primeiramente, observamos que as órbitas periódicas de $\widetilde{X}\left(f+\mu_{0}+\right.$ $\left.\mu_{1} y\right)$ em $C_{ \pm \frac{\pi}{2}}$ não dependem de $\mu_{0}$. Além disso, a segunda derivada da transformação de Poincaré dessas órbitas periódicas é da forma

$\pi^{\prime \prime}= \pm 2 \int_{0}^{\tau} a_{1}(s) d s+2 \mu_{1} \tau$.

Segue que o conjunto $B_{4}^{1}(X(f))$ de parâmetros $\mu_{1}$, para os quais as órbitas periódicas em $C_{ \pm \frac{\pi}{2}}$ não são semi-estáveis (isto é, $\pi^{\prime \prime}=0$ ), é finito. Portanto, $B_{4}^{1}(X(f))$ tem medida de Lebesgue zero em $\Re$.

Lembramos a propriedade 3 da definição de $\Sigma^{1}$ : " $X(f) \in \Sigma^{1}(M)$ não tem nenhuma conexão de selas".

Lema 3.4 Dados $X(f) \in \mathcal{E}^{1, r}(M), r \geq 1$ e $\mu_{0} \notin B_{1}^{1}(X(f))$. Então, o conjunto

$B_{5}^{1}\left(X(f) ; \mu_{0}\right)=\left\{\mu_{1} \in \Re: \tilde{X}\left(f+\mu_{0}+\mu_{1} y\right)\right.$ não tem a propriedade 3 de $\left.\Sigma^{1}\right\}$

tem medida de Lebesgue zero em $\Re$. 
Prova. Fixamos $\mu_{0} \notin B_{1}(X(f))$. O número de singularidades de $\widetilde{X}(f+$ $\left.\mu_{0}\right)$ é finito, por conseguinte, as conexões de selas de $\widetilde{X}_{\mu}(f)$ também acontecem em número finito de valores do parâmetro $\mu_{1}$.

Afirmamos que todas as conexões podem ser quebradas com perturbações da forma $\widetilde{X}\left(f+\mu_{0}+\mu_{1} y\right)$.

Suponhamos que para $\mu_{1}^{*}, \tilde{X}\left(f+\mu_{0}+\mu_{1}^{*} y\right)$ tenha uma trajetória $\widehat{p q}$ na parte superior de $\widetilde{M}$ conectando as selas $p$ e $q$ em $C_{0}$. Indicamos por $S_{E}(q)$ a separatriz estável de $q$ e por $S_{I}(p)$ a separatriz instável de $p$. Ver figura 3.1.

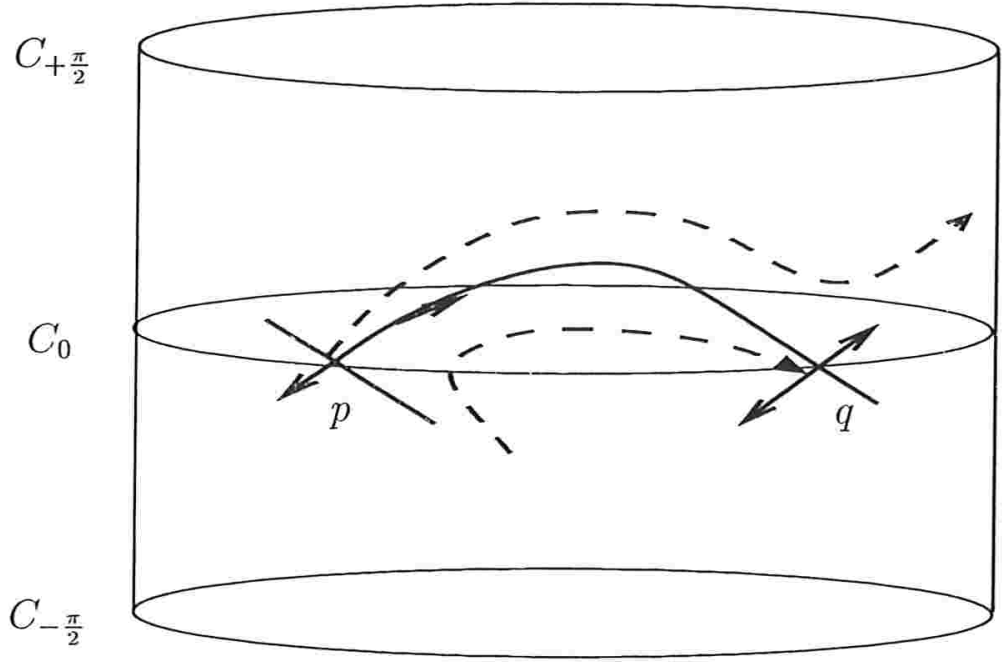

a)

Figura 3.1: Desconexões das selas $p$ e $q$ para $\mu_{1}>\mu_{1}^{*}$

Usamos a fórmula da integral de Sotomayor-Melnikov para calcular a derivada da função separação das variedades estáveis e instáveis das selas de $\widetilde{X}\left(f+\mu_{0}+\mu_{1} y\right)$. A função separação é definida em uma seção transversal à trajetória que liga as selas. Para maiores detalhes sobre a integral, ver $[7,13]$.

Se denotamos por $\operatorname{sep}\left(\mu_{1}\right)$ a função de separação das variedades $S_{I}(p, \widetilde{X}(f+$ $\left.\mu_{0}+\mu_{1} y\right)$ e $S_{E}\left(q, \tilde{X}\left(f+\mu_{0}+\mu_{1} y\right)\right.$, então, a derivada de $\operatorname{sep}\left(\mu_{1}\right)$ com respeito ao parâmetro $\mu_{1}$ é da forma

$$
\begin{aligned}
\operatorname{sep}_{\mu_{1}}\left(\mu_{1}^{*}\right)= & \int_{-\infty}^{+\infty} \exp \left(-\int_{0}^{t} \operatorname{div}\left(\tilde{X}\left(f+\mu_{0}+\mu_{1}^{*} y\right)(u(s), v(s))\right) d s\right) \\
& \cdot \widetilde{X}\left(f+\mu_{0}+\mu_{1}^{*} y\right) \wedge \frac{d}{d \mu_{1}} \tilde{X}\left(f+\mu_{0}+\mu_{1}^{*} y\right)(u(s), v(s)) d t
\end{aligned}
$$


onde $\wedge$ é o produto de vetores definido como segue

$\left(v_{1}, v_{2}\right) \wedge\left(w_{1}, w_{2}\right)=-\operatorname{det}\left(\begin{array}{cc}v_{1} & v_{2} \\ w_{1} & w_{2}\end{array}\right)$ e $(u(s), v(s))$ é a órbita que conecta as selas $p$ e $q$.

Em nosso caso, conseguimos

$$
\begin{gathered}
\operatorname{sep}_{\mu_{1}}(0)=-\int_{-\infty}^{+\infty} \exp \left(-\int_{0}^{t} d i v\left(\tilde{X}\left(f+\mu_{0}+\mu_{1}^{*} y\right)(u(s), v(s))\right) d s\right) \\
\cdot \sin ^{2}(v(t)) \cos ^{2}(v(t)) d t
\end{gathered}
$$

Observamos que o integrando em (3.2) é uma função não-negativa. Pois $v(t) \in\left[-\frac{\pi}{2}, \frac{\pi}{2}\right]$. Portanto, $\operatorname{sep}_{\mu_{1}}(0)<0$ e a conexão das selas $p$ e $q$ é quebrada sem que possa surgir nenhuma outra conexão. Este fato segue de considerar que a perturbação dada pelo parâmetro $\mu_{1}$ sempre aponta na direção $y$.

Concluímos que $B_{5}^{1}\left(X(f) ; \mu_{0}\right)$ é um conjunto discreto de parâmetros $\mu_{1}$ tais que $\tilde{X}\left(f+\mu_{0}+\mu_{1} y\right)$ tem alguma conexão de selas. Portanto $B_{5}^{1}\left(X(f) ; \mu_{0}\right)$ tem medida de Lebesgue nula em $\Re$.

Prova do Teorema 1. O conjunto $B^{1}(X(f))$ é a união dos seguintes conjuntos

$$
\begin{gathered}
\mathcal{B}_{1}=B_{1}^{1}(X(f)) \times \Re, \\
\mathcal{B}_{2}=\bigcup_{\left.\mu_{0} \in \Re-B_{1}^{1}(X(f))\right)}\left\{\mu_{0}\right\} \times B_{2}^{1}\left(X(f), \mu_{0}\right), \\
\mathcal{B}_{3}=\bigcup_{\mu_{0} \in \Re}\left\{\mu_{0}\right\} \times B_{3}^{1}\left(X(f) ; \mu_{0}\right), \\
\mathcal{B}_{4}=\Re \times B_{4}^{1}(X(f))
\end{gathered}
$$

e

$$
\mathcal{B}_{5}=\bigcup_{\left.\mu_{0} \in \Re-B_{1}^{1}(X(f))\right)}\left\{\mu_{0}\right\} \times B_{5}^{1}\left(X(f), \mu_{0}\right),
$$

onde $B_{i}^{1}(X(f), \cdot), i=1, \ldots, 5$, são dados pelos lemas $3.1,3.2,3.3,3.4$ respectivamente.

Cada conjunto $\mathcal{B}_{i}$ satisfaz as seguintes propriedades:

- Contém parâmetros $\left(\mu_{0}, \mu_{1}\right) \in \Re^{2}$ tais que o campo $X\left(f+\mu_{0}+\mu_{1} y\right)$ viola pelo menos uma das propriedades de $\Sigma^{1}$. 
- É mensurável. Pois seu complemento em $\Re^{2}$ é um conjunto aberto.

- A medida é zero em $\Re^{2}$. Pois, $\mathcal{B}_{1}$ e $\mathcal{B}_{4}$ são produtos de $\Re$ por um conjunto de medida nula em $\Re$. Para calcular a medida de $\mathcal{B}_{i}$ com $i=2,3$ e 5, aplicamos o Teorema de Fubini [10] como segue

$$
\int_{\Re^{2}} \chi\left(\mathcal{B}_{i}\right) d \mu_{0} d \mu_{1}=\int\left(\int \chi\left(\cdot, B_{i}^{1}\left(X(f), \mu_{0}\right)\right) d \mu_{1}\right) d \mu_{0}=0
$$

onde $\chi(\cdot)$ é a função característica de conjuntos em $\Re^{2}$ também chamada de indicadora.

Então, $B^{1}(X(f))$ é união de conjuntos de medida zero em $\Re^{2}$, portanto, é mensurável com medida de Lebesgue zero em $\Re^{2}$.

Teorema $2 \Sigma^{1}$ é aberto e denso em $\mathcal{E}^{1, r}(M)$ com $r \geq 2$.

Prova. A densidade de $\Sigma^{1}$ segue diretamente do Teorema 1. Pois, para cada $X(f) \in \mathcal{E}^{1, r}$ e $\forall \epsilon>0$ existe $\mu=\left(\mu_{0}, \mu_{1}\right) \in \Re^{2}$ tal que $\left|X_{\mu}(f)-X(f)\right|<\epsilon$, onde $X_{\mu}(f)=X\left(f+\mu_{0}+\mu_{1} y\right) \in \Sigma^{1}$.

Agora queremos ver que $\Sigma^{1}$ é aberto em $\mathcal{E}^{1, r}$.

Sejam $X(f)=y \frac{\partial}{\partial x}+\left(a_{0}(x)+a_{1}(x) y\right) \frac{\partial}{\partial y}$ e $X(g)=y \frac{\partial}{\partial x}+\left(b_{0}(x)+b_{1}(x) y\right) \frac{\partial}{\partial y}$. Pelas proposições 2.1 e 2.6, as singularidades de $\tilde{X}(f)$ são da forma $\left(u_{*}, 0\right) \in$ $C_{0}$ com $u_{*}$ raíz de $a_{0}(u)=0$. A hiperbolicidade dessas singularidades é dada pelas relações $\Delta X(f)\left(u_{*}, 0\right)=a_{0}^{\prime}\left(u_{*}\right) \neq 0$ e $\sigma X(f)\left(u_{*}, 0\right)=a_{1}\left(u_{*}\right) \neq 0$.

Assim, escolhendo $\mu_{0}$ e $\mu_{1}$ suficientemente pequenos, vemos que o campo $X(g)$ com funções coeficientes $b_{0}=a_{0}+\mu_{0}, b_{1}=a_{1}+\mu_{1}$ tem singularidades do mesmo tipo que $X(f)$.

Além disso, se $X(f)$ tem uma órbita periódica hiperbólica, o campo $X(g)$ com $b_{1}=a_{1}(u)+\mu_{1}$ para valores pequenos de $\mu_{1}$ terá uma órbita periódica hiperbólica. Também, a semi-estabilidade das órbitas periódicas em $C_{ \pm \frac{\pi}{2}}$ não é alterada por essas mesmas perturbações.

Então, é possível escolher uma vizinhança aberta, $\mathcal{U}$, de $X(f)$ em $\mathcal{E}^{1, r}$ tal que $\mathcal{U} \subset \Sigma^{1}$.

\subsection{Caso $n=2$.}

\subsubsection{Definição de $\Sigma^{2}$}

Vimos na proposição 2.4 que o campo $\widetilde{X}(f)$ tem órbitas periódicas nos círculos $C_{ \pm \frac{\pi}{2}}$. 
As órbitas $\gamma_{ \pm}(u)=\left(\phi(u), \pm \frac{\pi}{2}\right)$ com $u \in S^{1}$ e período $\tau$ têm estabilidade $\chi=\mp \int_{0}^{\tau} a_{2}(u) d u$.

Definição 3.2 Definimos $\Sigma^{2}$ como o conjunto dos campos $X(f) \in \mathcal{E}^{2, r}, r \geq$ 1 , tais que suas compactificações $\widetilde{X}(f)$ têm :

1. as singularidades hiperbólicas e contidas no círculo $C_{0}$;

2. as órbitas periódicas hiperbólicas e contidas em $\widetilde{M}$;

3. nenhuma conexão de selas.

A observação 3.1.1, também se aplica aos campos $X(f) \in \mathcal{E}^{2, r}$.

\subsubsection{Genericidade de $\Sigma^{2}$}

Seja $\mu=\left(\mu_{0}, \mu_{1}, \mu_{2}\right) \in \Re^{3}$.

Teorema 3 Dado $X(f) \in \mathcal{E}^{2, r}(M), r \geq 1$. Então, o conjunto

$$
B^{2}(X(f))=\left\{\mu \in \Re^{3}: X\left(f+\mu_{0}+\mu_{1} y+\mu_{2} y^{2}\right) \notin \Sigma^{2}(M)\right\}
$$

é um subconjunto de medida de Lebesgue zero em $\Re^{3}$.

A prova do Teorema 3 segue de vários lemas.

Lema 3.5 Dado $X(f) \in \mathcal{E}^{2, r}(M)$ com $r \geq 1$. Então, o conjunto

$$
\begin{gathered}
B_{1}^{2}(X(f))=\left\{\mu_{0} \in \Re: \widetilde{X}\left(f+\mu_{0}\right)\right. \text { tem alguma singularidade } \\
\left.\left(u_{*}, 0\right) \in C_{0} \text { com } \Delta \widetilde{X}\left(f+\mu_{0}\right)=0\right\}
\end{gathered}
$$

tem medida de Lebesgue zero em $\Re$.

Prova. Ver prova do Lema 3.1.

Lema 3.6 Dados $X(f) \in \mathcal{E}^{2, r}(M), r \geq 1$ e $\mu_{0} \notin B_{1}^{2}(X(f))$. Então, o conjunto

$$
\begin{aligned}
B_{2}^{2}\left(X(f) ; \mu_{0}\right)= & \left\{\mu_{1} \in \Re: \tilde{X}\left(f+\mu_{0}+\mu_{1} y\right)\right. \text { tem alguma } \\
& \text { singularidade nấo-hiperbólica }\}
\end{aligned}
$$

tem medida de Lebesgue zero em $\Re$.

Prova. Ver prova do Lema 3.2. 
Lema 3.7 Dado $X(f) \in \mathcal{E}^{2, r}(M), r \geq 1$. Para $\mu_{0} \in \Re$, o conjunto

$$
\begin{array}{r}
B_{3}^{2}\left(X(f) ; \mu_{0}\right)=\left\{\mu_{1} \in \Re: \widetilde{X}\left(f+\mu_{0}+\mu_{1} y\right)\right. \text { tem alguma órbita } \\
\text { periódica não-hiperbólica }\}
\end{array}
$$

tem medida de Lebesgue zero em $\Re$.

Prova. Ver prova do Lema 3.3 parte $i$ ).

Lema 3.8 Dado $X(f) \in \mathcal{E}^{2, r}(M) \operatorname{com} f(x, y)=a_{0}(x)+a_{1}(x) y+a_{2}(x) y^{2}, e$ $r \geq 1$. Então, o conjunto

$$
\begin{aligned}
& B_{4}^{2}(X(f))=\left\{\mu_{2} \in \Re: \widetilde{X}\left(f+\mu_{2} y^{2}\right)\right. \text { tem alguma órbita } \\
& \text { periódica não-hiperbólica em } \left.C_{ \pm \frac{\pi}{2}}\right\}
\end{aligned}
$$

tem medida de Lebesgue zero em $\Re$.

Prova. Pela Proposição 2.4, o campo $\widetilde{X}(f)$ tem duas órbitas periódicas de período $\tau$ (igual ao período das funções $a_{i}(u)$ ) nos círculos $C_{ \pm \frac{\pi}{2}}$ com estabilidade $\chi=\mp \int_{0}^{\tau} a_{2}(u) d u$. Logo, $\widetilde{X}\left(f+\mu_{2} y^{2}\right)$ tem órbitas periódicas em $C_{ \pm \frac{\pi}{2}}$ não-hiperbólicas se e somente se $\mu_{2}= \pm \frac{1}{\tau} \int_{0}^{\tau} a_{2}(u) d u$.

Segue, então, que $B_{4}^{2}(X(f))$ é discreto e portanto de medida nula em $\Re$.

Observação 3.2.1 Para todo $\left(\mu_{0}, \mu_{1}\right) \in \Re^{2}, B_{4}^{2}\left(X\left(f+\mu_{0}+\mu_{1} y\right)\right)=B_{4}^{2}(X(f))$.

Lembramos a propriedade 3 da definição de $\Sigma^{2}$ : " $X(f) \in \Sigma^{2}(M)$ não tem nenhuma conexão de selas".

Lema 3.9 Dados $X(f) \in \mathcal{E}^{2, r}(M), r \geq 1$ e $\mu_{0} \notin B_{1}^{2}(X(f))$. Então, o conjunto

$B_{5}^{2}\left(X(f) ; \mu_{0}\right)=\left\{\mu_{1} \in \Re: \widetilde{X}\left(f+\mu_{0}+\mu_{1} y\right)\right.$ não tem a propriedade 3 de $\left.\Sigma^{2}\right\}$ tem medida de Lebesgue zero em $\Re$.

\section{Prova.}

Ver a prova do Lema 3.4.

Prova do Teorema 3. O conjunto $B^{2}(X(f))$ é a união dos seguintes conjuntos: 


$$
\begin{gathered}
\mathcal{B}_{1}=B_{1}^{2}(X(f)) \times \Re^{2}, \\
\mathcal{B}_{2}=\bigcup_{\mu_{0} \in \Re-B_{1}^{2}(X(f))}\left\{\mu_{0}\right\} \times B_{2}^{1}\left(X(f), \mu_{0}\right) \times \Re, \\
\mathcal{B}_{3}=\bigcup_{\mu_{0} \in \Re}\left\{\mu_{0}\right\} \times B_{3}^{2}\left(X(f) ; \mu_{0}\right) \times \Re, \\
\mathcal{B}_{4}=\Re^{2} \times B_{4}^{2}(X(f)),
\end{gathered}
$$

e

$$
\mathcal{B}_{5}=\bigcup_{\mu_{0} \in \Re-B_{1}^{1}(X(f))}\left\{\mu_{0}\right\} \times B_{4}^{1}\left(X(f), \mu_{0}\right) \times \Re,
$$

onde $B_{i}^{2}(X(f), \cdot), i=1, \ldots, 5$, são dados pelos lemas 3.5, 3.6, 3.7, 3.8, 3.9 respectivamente.

Cada conjunto $\mathcal{B}_{i}$ satisfaz as seguintes propriedades:

- Contém parâmetros $\left(\mu_{0}, \mu_{1}, \mu_{2}\right) \in \Re^{3}$ tais que o campo $X\left(f+\mu_{0}+\mu_{1} y+\mu_{2} y^{2}\right)$ viola pelo menos uma das propriedades de $\Sigma^{2}$.

- É mensurável. Pois seu complemento em $\Re^{3}$ é um conjunto aberto.

- Tem medida zero em $\Re^{3}$. Pois, $\mathcal{B}_{1}$ e $\mathcal{B}_{4}$ são produtos de $\Re^{2}$ por um conjunto de medida nula em $\Re$. Para calcular a medida de $\mathcal{B}_{i}$ com $i=2,3$ e 5 , aplicamos o Teorema de Fubini como segue

$$
\int_{\Re^{3}} \chi\left(\mathcal{B}_{i}\right) d \mu_{0} d \mu_{1} d \mu_{2}=\int_{\Re^{2}}\left(\int \chi\left(\cdot, B_{i}^{1}\left(X(f), \mu_{0}\right), \cdot\right) d \mu_{1}\right) d \mu_{0} d \mu_{2}=0
$$

onde $\chi(\cdot)$ é a função característica de conjuntos em $\Re^{3}$, também chamada de indicadora.

Então, $B^{2}(X(f))$ é união de conjuntos de medida zero em $\Re^{3}$, portanto, é mensurável com medida de Lebesgue zero em $\Re^{3}$.

Apresentamos a seguir o Teorema de genericidade de $\Sigma^{2}$.

Teorema $4 \Sigma^{2}$ é aberto e denso em $\mathcal{E}^{2, r}(M)$ com $r \geq 1$.

Prova. A densidade de $\Sigma^{2}$ segue diretamente do Teorema 3.

Para ver que $\Sigma^{2}$ é aberto em $\mathcal{E}^{2, r}$, seguimos a mesma linha da prova do Teorema de genericidade para $n=1$. Neste caso, devemos ter em conta que se $X(f)$ tem órbitas periódicas hiperbólicas nos círculos $C_{ \pm \frac{\pi}{2}}$, o campo $X\left(f+\mu_{0}+\mu_{1} y+\mu_{2} y^{2}\right)$, para valores pequenos de $\mu_{2}$, terá uma órbita periódica hiperbólica, também. 


\subsection{Caso $n=3$}

Lembramos que as trajetórias de $X(f)$ que escapam ao infinito são transversais ou tangentes aos círculos $C_{ \pm \frac{\pi}{2}}$ em pontos isolados, como vimos na proposição 2.5 .

\subsubsection{Definição de $\Sigma^{3}$}

Definição 3.3 Definimos $\Sigma^{3}$ como o conjunto formado pelos campos $X(f) \in$ $\mathcal{E}^{3, r}(M), r \geq 1$, tais que $\widetilde{X}(f)$ tem:

1. as singularidades hiperbólicas e contidas em $C_{0}$;

2. as órbitas periódicas hiperbólicas e contidas no int $(\widetilde{M})$.

3. as tangências de trajetórias com $C_{ \pm \frac{\pi}{2}}$ de tipo parabólico.

4. (a) Nenhuma conexão de selas.

(b) As separatrizes de pontos singulares em $C_{0}$ transversais a $C_{ \pm \frac{\pi}{2}}$.

(c) As trajetórias tangentes a $C_{ \pm \frac{\pi}{2}}$ no máximo em um ponto.

\subsubsection{Genericidade de $\Sigma^{3}$}

Teorema 5 Dado $X(f) \in \mathcal{E}^{3, r}(M), r \geq 1$. Então, o conjunto

$$
B^{3}(X(f))=\left\{\left(\mu_{0}, \mu_{1}, \mu_{2}\right) \in \Re^{3}: X\left(f+\mu_{0}+\mu_{1} y+\mu_{2} y^{3}\right) \notin \Sigma^{3}(M)\right\}
$$

é um subconjunto de medida de Lebesgue zero em $\Re^{3}$.

A prova do Teorema 5 segue dos seguintes lemas.

Lema 3.10 Dado $X(f) \in \mathcal{E}^{3, r}(M), r \geq 1$. Então, o conjunto

$$
\begin{gathered}
B_{1}^{3}(X(f))=\left\{\mu_{0} \in \Re: \tilde{X}\left(f+\mu_{0}\right)\right. \text { tem alguma singularidade } \\
\left.\left(u_{*}, 0\right) \in C_{0} \text { com } \Delta \widetilde{X}\left(f+\mu_{0}\right)=0\right\}
\end{gathered}
$$

tem medida de Lebesgue zero em $\Re$.

Prova. A prova é similar ao Lema 3.1. 
Lema 3.11 Dados $X(f) \in \mathcal{E}^{3, r}(M), r \geq 1$ e $\mu_{0} \notin B_{1}^{3}(X(f))$. Então, o conjunto

$$
\begin{aligned}
B_{2}^{3}\left(X(f) ; \mu_{0}\right)= & \left\{\mu_{1} \in \Re: \tilde{X}\left(f+\mu_{0}+\mu_{1} y\right)\right. \text { tem alguma } \\
& \text { singularidade não-hiperbólica }\}
\end{aligned}
$$

tem medida de Lebesgue zero em $\Re$.

Prova. A prova é similar ao Lema 3.2.

Lema 3.12 Dado $X(f) \in \mathcal{E}^{3, r}(M), r \geq 1$. Para $\mu_{0} \in \Re$, o conjunto

$$
\begin{aligned}
& B_{3}^{3}\left(X(f) ; \mu_{0}\right)=\left\{\mu_{1} \in \Re: \widetilde{X}\left(f+\mu_{0}+\mu_{1} y\right)\right. \text { tem alguma órbita } \\
& \text { periódica não-hiperbólica }\}
\end{aligned}
$$

tem medida de Lebesgue zero em $\Re$.

Prova. A prova é similar ao Lema 3.3.

Lembramos a propriedade 3 de $\Sigma^{3}$ : "As tangências de trajetórias de $X(f) \in \Sigma^{3} \operatorname{com} C_{ \pm \frac{\pi}{2}}$ são parabólicas".

Lema 3.13 Dado $X(f) \in \mathcal{E}^{3, r}(M), r \geq 1$. Então, o conjunto

$$
\begin{aligned}
B_{4}^{3}(X(f))= & \left\{\mu_{2} \in \Re: X\left(f+\mu_{2} y^{3}\right)\right. \text { não tem a } \\
& \text { propriedade } \left.3 \text { de } \Sigma^{3}\right\}
\end{aligned}
$$

tem medida de Lebesgue zero em $\Re$.

Prova. Seja $X(f) \operatorname{com} f(x, y)=\sum_{i=0}^{3} a_{i}(x) y^{i}$. Pela proposição 2.5, o campo $\tilde{X}\left(f+\mu_{2} y^{3}\right)$ tem $\left(u_{*}, \pm \frac{\pi}{2}\right) \in C_{ \pm \frac{\pi}{2}}$ como ponto de tangência se $a_{3}\left(u_{*}\right)+\mu_{2}=0$ e mais ainda a tangência é parabólica se $a_{3}^{\prime}\left(u_{*}\right) \neq 0$.

O conjunto de valores críticos de $-a_{3}(u)$,

$$
\operatorname{Crit}\left(-a_{3}(u)\right)=\left\{\mu_{2} \in \Re: \exists\left(u_{*}, 0\right) \text { tal que } a_{3}\left(u_{*}\right)+\mu_{2}=0 \text { e } a_{3}^{\prime}\left(u_{*}\right)=0\right\},
$$

descreve o conjunto de valores de $\mu_{2} \in \Re$ tais que $X\left(f+\mu_{0}+\mu_{1} y+\mu_{2} y^{3}\right)$ não satisfaz a propriedade 3 de $\Sigma^{3}$.

Então, pelo lema de Sard 1.1, $B_{4}^{3}\left(X(f) ; \mu_{0}, \mu_{1}\right)$ tem medida de Lebesgue zero em $\Re$.

Observação 3.3.1 $X(f)$ e $X\left(f+\mu_{0}+\mu_{1} y\right)$ têm os mesmos pontos de tangências em $C_{ \pm \frac{\pi}{2}}$. 
Lembramos a propriedade 4 de $\Sigma^{3}$ : " $X(f) \in \Sigma^{3}$ se

1. não tem nenhuma conexão de selas.

2. tem as separatrizes de pontos singulares em $C_{0}$ transversais a $C_{ \pm \frac{\pi}{2}}$.

3. tem as trajetórias tangentes a $C_{ \pm \frac{\pi}{2}}$ no máximo em um ponto."

Lema 3.14 Dados $X(f) \in \mathcal{E}^{3, r}(M), r \geq 1, \mu_{0} \notin B_{1}^{3}(X(f))$ e $\mu_{2} \notin B_{4}^{3}(X(f))$. Então, o conjunto

$$
\begin{gathered}
B_{5}^{3}\left(X(f) ; \mu_{0}, \mu_{2}\right)=\left\{\mu_{1} \in \Re: X\left(f+\mu_{0}+\mu_{1} y+\mu_{2} y^{3}\right)\right. \text { não tem } \\
\text { a propriedade } \left.4 \text { de } \Sigma^{3}\right\}
\end{gathered}
$$

tem medida de Lebesgue zero em $\Re$.

Prova. Fixamos $\mu_{0} \notin B_{1}^{3}(X(f))$ e $\mu_{2} \notin B_{3}^{3}(X(f))$ como nos lemas 3.10 e 3.13

As conexões de selas de $\widetilde{X}\left(f+\mu_{0}+\mu_{1} y+\mu_{2} y^{3}\right)$ devem acontecer em um número finito de valores do parâmetro $\mu_{1}$, pois o número de singularidades é finito.

Basicamente, temos três situações em que $\tilde{X}(f)$ tem separatrizes conectando singularidades em $C_{0}$ ou pontos de tangência em $C_{ \pm \frac{\pi}{2}}$.

a) Conexões de selas pertencentes a $C_{0}$.

b) Uma separatriz de sela tangente a $C_{+\frac{\pi}{2}}$ ou $C_{-\frac{\pi}{2}}$ com tangência parabólica.

c) Uma órbita com duas tangências parabólicas a $C_{ \pm \frac{\pi}{2}}$. As tangências podem estar em círculos $C_{ \pm \frac{\pi}{2}}$ diferentes.

$\mathrm{Na}$ figura 3.2 , mostramos as situações $a$ ), $b$ ) e $c$ ).

Todas essas conexões de selas ou de tangências acontecem em um número finito de valores do parâmetro $\mu_{1}$. A análise feita no lema 3.4 se aplica diretamente as situações $a$ ), $b$ ) e $c$ ), ainda no caso em que uma órbita tenha duas tangências parabólicas em um mesmo círculo $C_{ \pm \frac{\pi}{2}}$.

De fato, podemos quebrar essas conexões com perturbações da forma $\tilde{X}\left(f+\mu_{0}+\mu_{1} y+\mu_{2} y^{3}\right)$ para $\mu_{0}$ e $\mu_{2}$ fixos. A integral de Sotomayor-Melnikov nos permite calcular a derivada da função separação das variedades estáveis e instáveis das selas conectadas, da seguinte forma

$$
\begin{gathered}
\operatorname{sep}_{\mu_{1}}(0)=-\int_{-\infty}^{+\infty} \exp \left(-\int_{0}^{t} \operatorname{div}\left(\tilde{X}\left(f+\mu_{0}+\mu_{1}^{*} y+\mu_{2} y^{3}\right)(u(s), v(s))\right) d s\right) \\
\cdot \sin ^{2}(v(t)) \cos ^{2}(v(t)) d t
\end{gathered}
$$




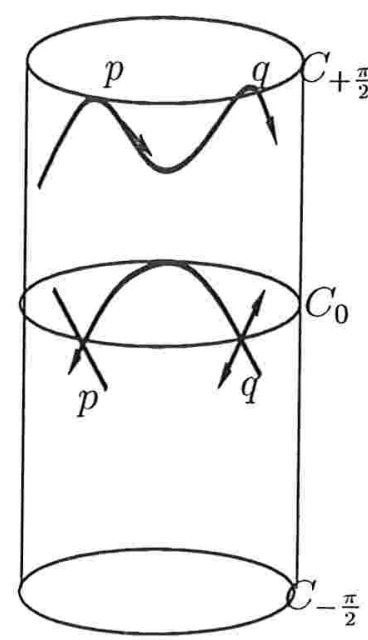

a)

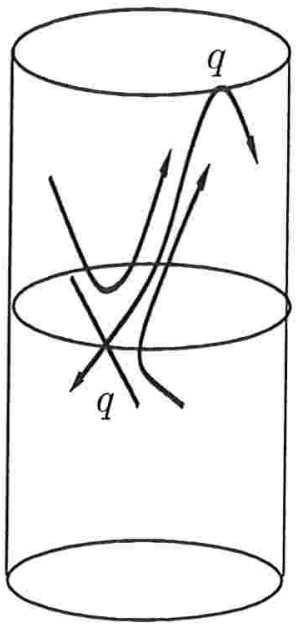

b)

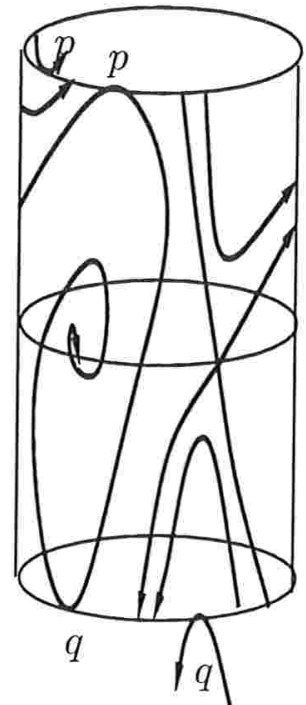

c)

Figura 3.2: Tipos de conexões de selas em $C_{0}$ e de pontos de tangência em $C_{ \pm \frac{\pi}{2}}$.

Nos casos $b$ ) e $c$ ) devemos considerar o tempo de percurso das trajetórias tangentes aos círculos $C_{ \pm \frac{\pi}{2}}$. Então, a derivada da função separação das trajetórias tangentes a $C_{ \pm \frac{\pi}{2}}$ tem a forma

$$
\begin{gathered}
\operatorname{sep}_{\mu_{1}}(0)=-\frac{1}{|\widetilde{X}(f)(q)|} \int_{0}^{T} \exp \left(-\int_{0}^{t} \operatorname{div}\left(\tilde{X}\left(f+\mu_{0}+\mu_{1}^{*} y+\mu_{2} y^{3}\right)(u(s), v(s))\right) d s\right) \\
\cdot \sin ^{2}(v(t)) \cos ^{2}(v(t)) d t .
\end{gathered}
$$

onde $T$ é o tempo de percurso da trajetória tangente entre um ponto $q \in$ $\operatorname{int}(\widetilde{M})$ e o ponto de tangência $p \in C_{ \pm \frac{\pi}{2}}$.

Os integrandos em (3.3) e em (3.4) são funções não-negativas para $v(t) \epsilon$ $\left[-\frac{\pi}{2}, \frac{\pi}{2}\right]$. Portanto, $\operatorname{sep}_{\mu_{1}}(0)<0$ e as conexões são quebradas sem que possa surgir nenhuma outra conexão. Este fato segue de considerar que a perturbação dada pelo parâmetro $\mu_{1}$ sempre aponta na direção $y$. Ver figura 3.3.

Concluímos que $B_{4}\left(X(f) ; \mu_{0}, \mu_{2}\right)$ é um subconjunto discreto de $\Re$ e portanto tem medida de Lebesgue nula. 


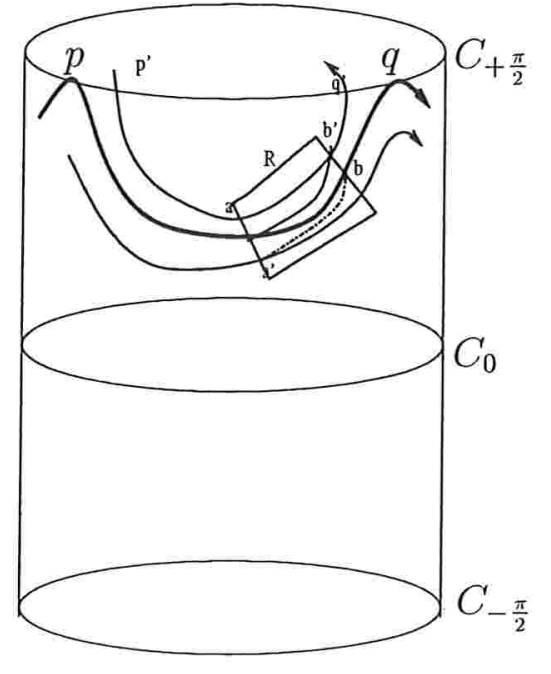

a)

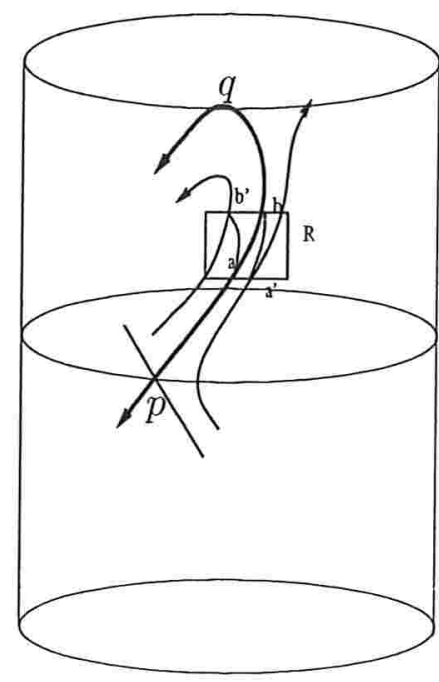

b)

Figura 3.3: Desconexões de separatrizes

Prova do Teorema 5. O conjunto $B^{3}(X(f))$ é a união dos seguintes conjuntos

$$
\begin{gathered}
\mathcal{B}_{1}=B_{1}^{3}(X(f)) \times \Re^{2}, \\
\mathcal{B}_{2}=\bigcup_{\mu_{0} \in \Re-B_{1}^{3}(X(f))}\left\{\mu_{0}\right\} \times B_{2}^{3}\left(X(f), \mu_{0}\right) \times \Re, \\
\mathcal{B}_{3}=\bigcup_{\mu_{0} \in \Re}\left\{\mu_{0}\right\} \times B_{3}^{3}\left(X(f) ; \mu_{0}\right) \times \Re, \\
\mathcal{B}_{4}=\Re^{2} \times B_{4}^{3}(X(f)),
\end{gathered}
$$

$\mathrm{e}$

$$
\mathcal{B}_{5}=\bigcup_{\left(\mu_{0}, \mu_{2}\right) \in\left(\Re-B_{1}^{3}(X(f))\right) \cup\left(\Re-B_{4}^{3}(X(f))\right)}\left\{\mu_{0}\right\} \times B_{5}^{3}\left(X(f), \mu_{0}, \mu_{2}\right) \times\left\{\mu_{2}\right\},
$$

onde $B_{i}^{3}(X(f), \cdot), i=1, \ldots, 5$, são dados pelos lemas $3.10,3.11,3.12,3.13 \mathrm{e}$ 3.14 respectivamente.

Cada conjunto $\mathcal{B}_{i}$ satisfaz as seguintes condições:

- Contém parâmetros $\left(\mu_{0}, \mu_{1}, \mu_{2}\right) \in \Re^{3}$ tais que o campo $X\left(f+\mu_{0}+\mu_{1} y+\mu_{2} y^{3}\right)$ viola pelo menos uma das propriedades de $\Sigma^{3}$. 
- É mensurável. Pois seu complemento em $\Re^{3}$ é um conjunto aberto.

- Tem medida zero em $\Re^{3}$. Pois, $\mathcal{B}_{1}$ e $\mathcal{B}_{4}$ são produtos de $\Re^{2}$ por um conjunto de medida nula em $\Re$. Para calcular a medida de $\mathcal{B}_{i}$ com $i=2,3$ e 5, aplicamos o Teorema de Fubini como segue

$$
\begin{aligned}
& \int_{\Re^{3}} \chi\left(\mathcal{B}_{2}\right) d \mu_{0} d \mu_{1} d \mu_{2}=\int_{\Re^{2}}\left(\int_{\Re} \chi\left(\cdot, B_{2}^{3}(X(f), \cdot), \cdot\right) d \mu_{1}\right) d \mu_{0} d \mu_{2}=0 \\
& \int_{\Re^{3}} \chi\left(\mathcal{B}_{3}\right) d \mu_{0} d \mu_{1} d \mu_{2}=\int_{\Re^{2}}\left(\int_{\Re} \chi\left(\cdot, B_{3}^{3}(X(f), \cdot), \cdot\right) d \mu_{1}\right) d \mu_{0} d \mu_{2}=0 \\
& \int_{\Re^{3}} \chi\left(\mathcal{B}_{5}\right) d \mu_{0} d \mu_{1} d \mu_{2}=\int_{\Re^{2}}\left(\int_{\Re} \chi\left(\cdot, B_{5}^{3}(X(f), \cdot, \cdot), \cdot\right) d \mu_{1}\right) d \mu_{0} d \mu_{2}=0
\end{aligned}
$$

onde $\chi(\cdot)$ é a função característica de conjuntos em $\Re^{3}$, também chamada de função indicadora.

Então, $B^{3}(X(f))$ é união de conjuntos de medida zero em $\Re^{3}$, portanto, é mensurável com medida de Lebesgue zero em $\Re^{3}$.

Apresentamos a seguir o Teorema de genericidade de $\Sigma^{3}$.

Teorema $6 \Sigma^{3}$ é aberto e denso em $\mathcal{E}^{3, r}(M)$ com $r \geq 1$.

Prova. A densidade de $\Sigma^{3}$ segue diretamente do Teorema 5. Para cada $X(f) \in \mathcal{E}^{3, r}$ e $\forall \epsilon>0$ existe $\mu=\left(\mu_{0}, \mu_{1}, \mu_{2}\right) \in \Re^{3}$ tal que $\left|X_{\mu}(f)-X(f)\right|<\epsilon$ onde $X_{\mu}(f)=X\left(f+\mu_{0}+\mu_{1} y+\mu_{2} y^{3}\right) \in \Sigma^{3}$.

Agora, queremos ver que $\Sigma^{3}$ é aberto em $\mathcal{E}^{3}$.

Sejam $X(f)=y \frac{\partial}{\partial x}+\sum_{i=0}^{3} a_{i}(x) y^{i} \frac{\partial}{\partial y}$ e $X(g)=y \frac{\partial}{\partial x}+\sum_{i=0}^{3} b_{i}(x) y^{i} \frac{\partial}{\partial y}$. Pelas proposições 2.1 e 2.6 , as singularidades de $\tilde{X}(f)$ são da forma $\left(u_{*}, 0\right) \in C_{0}$ com $u_{*}$ raíz de $a_{0}(u)=0$. A hiperbolicidade dessas singularidades é dada pelas relações $\Delta X(f)\left(u_{*}, 0\right)=a_{0}^{\prime}\left(u_{*}\right) \neq 0$ e $a_{1}\left(u_{*}\right) \neq 0$.

Os pontos de tangência parabólica são dados pelas condições $a_{3}\left(u_{*}\right)=0$ e $a_{3}^{\prime}\left(u_{*}\right) \neq 0$.

Assim, escolhendo $\mu_{0}, \mu_{1}$ e $\mu_{2}$ suficientemente pequenos, vemos que o campo $X(g)$ com funções coeficientes $b_{0}=a_{0}+\mu_{0}, b_{1}=a_{1}+\mu_{1}$ e $b_{3}=a_{3}+\mu_{2}$ tem singularidades e pontos de tangências do mesmo tipo que $X(f)$.

Além disso, se $X(f)$ tem uma órbita periódica hiperbólica, o campo $X(g)$ com $b_{1}=a_{1}(u)+\mu_{1}$ para valores pequenos de $\mu_{1}$ terá uma órbita periódica hiperbólica.

Então, é possível escolher uma vizinhança aberta, $\mathcal{U}$, de $X(f)$ em $\mathcal{E}^{3, r}$ tal que $\mathcal{U} \subset \Sigma^{3}$. 


\subsection{Caso $n=4$}

Dado $X(f) \in \mathcal{E}^{4, r}$ com $r \geq 1$. O campo compactificado $\widetilde{X}(f)$ tem singularidades nos círculos $C_{0}$ e $C_{ \pm \frac{\pi}{2}}$ como vimos na Proposição 2.6.

\subsubsection{Definição de $\Sigma^{4}$}

Definição 3.4 Definimos por $\Sigma^{4}$ o conjunto dos campos $X(f) \in \mathcal{E}^{4, r}$, com $r \geq 1$, tais que $\widetilde{X}(f)$ tem:

1. As singularidades $\left(u_{*}, \cdot\right) \in C_{0} \cup C_{ \pm \frac{\pi}{2}}$ hiperbólicas e os autovalores correspondentes às singularidades em $\mathrm{C}_{ \pm \frac{\pi}{2}}$ distintos.

2. As órbitas periódicas hiperbólicas e contidas no int $(\widetilde{M})$.

3. Nenhuma conexão de separatrizes de singularidades. Mais especificamente

(a) nenhuma conexão de selas pertencentes a $C_{0}$ ou a $C_{ \pm \frac{\pi}{2}}$,

(b) separatrizes de selas em $C_{0}$ ou em $C_{ \pm \frac{\pi}{2}}$ não são variedades fracas de nós em $C_{ \pm \frac{\pi}{2}}$.

Na figura 3.4, apresentamos os casos dos campos que não pertencem a $\Sigma^{4}$ por violar a condição 3. Em [4], esses campos são exibidos como que violam as condições B.3 e B.4.

\subsubsection{Genericidade de $\Sigma^{4}$.}

Teorema 7 Dado $X(f) \in \mathcal{E}^{4, r}(M), r \geq 1$. Então, o conjunto

$$
B^{4}(X(f))=\left\{\mu \in \Re^{4}: X\left(f+\mu_{0}+\mu_{1} y+\mu_{2} y^{3}+\mu_{3} y^{4}\right) \notin \Sigma^{4}(M)\right\}
$$

é um subconjunto de medida de Lebesgue zero em $\Re^{4}$.

A prova deste Teorema segue de vários lemas. O primeiro lema dá a medida do conjunto de campos que têm no mínimo uma singularidade nãohiperbólica em $\widetilde{M}$.

Lema 3.15 Dado $X(f)=X\left(\sum_{i=0}^{4} a_{i}(x) y^{i}\right) \in \mathcal{E}^{4, r}(M)$, com $r \geq 1$. Então,

a) o conjunto

$$
\begin{gathered}
B_{1}^{4}(X(f))=\left\{\mu_{0} \in \Re: \widetilde{X}\left(f+\mu_{0}\right)\right. \text { tem alguma singularidade em } \\
\left.C_{0} \text { com } \Delta \widetilde{X}\left(f+\mu_{0}\right)=0\right\}
\end{gathered}
$$

tem medida de Lebesgue zero em $\Re$. 


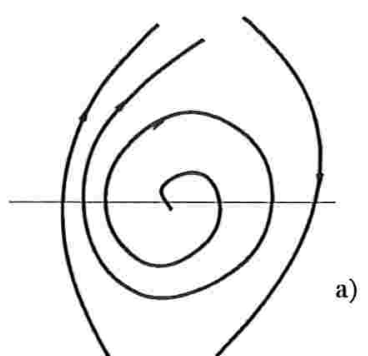

a)

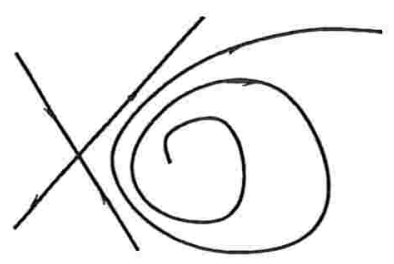

c)

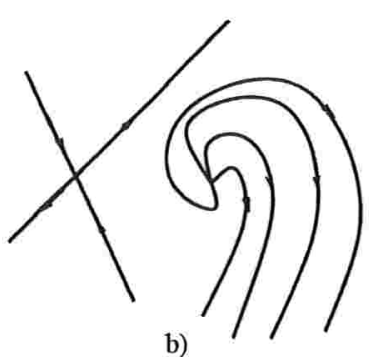

b)

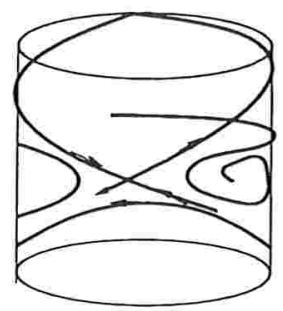

Figura 3.4: a) não cumpre B.3. b) e c) não cumprem B.4. d) a situação $c$ ) vista no cilindro compacto, não satisfaz a condição 3 da definição 3.4

b) o conjunto

$$
\begin{aligned}
& B_{2}^{4}(X(f))=\left\{\mu_{3} \in \Re: \tilde{X}\left(f+\mu_{3} y^{4}\right)\right. \text { tem alguma singularidade em } \\
& \left.C_{ \pm \frac{\pi}{2}} \operatorname{com} \Delta \widetilde{X}\left(f+\mu_{3} y^{4}\right)=0\right\}
\end{aligned}
$$

tem medida de Lebesgue zero em $\Re$

Prova. Os conjuntos $\operatorname{Crit}\left(-a_{0}\right)=\left\{\mu_{0}=-a_{0}(x): a_{0}^{\prime}(x)=0\right\} \mathrm{e}$ $\operatorname{Crit}\left(-a_{4}\right)=\left\{\mu_{3}=-a_{4}(x): a_{4}^{\prime}(x)=0\right\}$ determinam os conjuntos de parâmetros $\mu_{0}$ e $\mu_{3}$ tais que o campo $\widetilde{X}\left(f+\mu_{0}+\mu_{3} y^{4}\right)$ tem singularidades em $C_{0}$ e $C_{ \pm \frac{\pi}{2}}$ nas quais $\Delta \widetilde{X}\left(f+\mu_{0}+\mu_{3} y^{4}\right)=0$. Pelo Teorema de Sard, esses conjuntos têm medida de Lebesgue zero em $\Re$.

No seguinte lema, damos a medida do conjunto de campos que têm singularidades não-hiperbólicas em $\widetilde{M}$ quando $\Delta \neq 0$ e $\sigma=0$ ou, se são hiperbólicas em $C_{ \pm \frac{\pi}{2}}$, têm autovalores iguais.

Lema 3.16 Dados $X(f)=X\left(\sum_{i=0}^{4} a_{i}(x) y^{i}\right) \in \mathcal{E}^{4, r}(M)$ com $r \geq 1, \mu_{0} \notin$ $B_{1}^{4}(X(f))$ e $\mu_{3} \notin B_{2}^{4}(X(f))$. Então 
a) o conjunto

$$
\begin{aligned}
B_{3}^{4}\left(X(f) ; \mu_{0}\right)= & \left\{\mu_{1} \in \Re: \widetilde{X}\left(f+\mu_{0}+\mu_{1} y\right)\right. \text { tem alguma } \\
& \text { singularidade não-hiperbólica em } \left.C_{0}\right\}
\end{aligned}
$$

tem medida de Lebesgue zero em $\Re$.

b) o conjunto

$$
\begin{aligned}
B_{4}^{4}\left(X(f) ; \mu_{3}\right)=\{ & \left\{\mu_{2} \in \Re: \widetilde{X}\left(f+\mu_{2} y^{3}+\mu_{3} y^{4}\right)\right. \text { tem alguma } \\
& \text { singularidade hiperbólica com autovalores } \\
& \text { associados iguais ou não-hiperbólica em } \left.C_{ \pm \frac{\pi}{2}}\right\}
\end{aligned}
$$

tem medida de Lebesgue zero em $\Re$.

Prova. a) Para $\mu_{0} \notin B_{1}^{4}(X(f)), \Delta\left(\tilde{X}\left(f+\mu_{0}\right)\right)\left(u_{*}, 0\right) \neq 0$ com $\left(u_{*}, 0\right)$ singularidade de $\tilde{X}\left(f+\mu_{0}\right)$ em $C_{0}$. Notar que as singularidades de $\tilde{X}(f)$ são em números finitos, digamos $k$ singularidades em $C_{0}$.

Para cada $\left(x_{*, i}, 0\right) \in C_{0}$ singularidade de $\widetilde{X}\left(f+\mu_{0}+\mu_{1} y\right)$ existe um único valor $\mu_{1, i}^{*}$ tal que $\sigma\left(\tilde{X}\left(f+\mu_{0}+\mu_{1, i}^{*} y\right)\right)=0$. Pois $\sigma\left(\tilde{X}\left(f+\mu_{0}+\mu_{1}^{*} y\right)\right)=$ $a_{1}\left(x_{*, i}\right)+\mu_{1}$.

Segue facilmente que

$$
B_{3}^{4}\left(X(f) ; \mu_{0}\right) \text { é um conjunto discreto de } \Re \text {. }
$$

b) Dado $\mu_{3} \notin B_{2}^{4}(X(f))$. A condição

$$
a_{3}\left(u_{*}\right)+\mu_{2}=2 \sqrt{a_{4}^{\prime}\left(u_{*}\right)}
$$

implica que $\tilde{X}\left(f+\mu_{2} y^{3}+\mu_{3} y^{4}\right)$ tem uma singularidade $\left(u_{*}, \pm \frac{\pi}{2}\right)$ hiperbólica e com autovalores iguais. Então, existe um único valor $\mu_{2}^{*}$ que satisfaz a condição (3.5)

Por outro lado, para cada $\left(u_{*}, \pm \frac{\pi}{2}\right)$ singularidade não-hiperbólica de $\tilde{X}(f+$ $\left.\mu_{2} y^{3}+\mu_{3} y^{4}\right)$, existe um único valor $\mu_{2}^{*}$ tal que $\sigma\left(\widetilde{X}\left(f+\mu_{2}^{*} y^{3}+\mu_{3} y^{4}\right)\right)=$ $-\left(a_{3}\left(u_{*}\right)+\mu_{2}\right)=0$.

Dessas condições segue que o conjunto $B_{4}^{4}\left(X(f) ; \mu_{3}\right)$ é um subconjunto discreto de $\Re$ e portanto tem medida de Lebesgue zero em $\Re$.

A seguir, apresentamos a medida do conjunto de campos que têm no mínimo uma órbita periódica não-hiperbólica. Observamos que as órbitas periódicas de $\widetilde{X}_{\mu}(f)$ estão contidas no interior de $\widetilde{M}$. Isso nos permite usar o próprio campo $X_{\mu}(f)$. 
Lema 3.17 Dado $X(f) \in \mathcal{E}^{4, r}(M)$ com $r \geq 1$. Para $\mu_{0} \in \Re$, o conjunto

$$
\begin{array}{r}
B_{5}^{4}\left(X(f) ; \mu_{0}\right)=\left\{\mu_{1} \in \Re: \tilde{X}\left(f+\mu_{0}+\mu_{1} y\right)\right. \text { tem alguma órbita } \\
\text { periódica náo-hiperbólica }\}
\end{array}
$$

tem medida de Lebesgue zero em $\Re$.

Prova. A prova é idêntica a do Lema 3.3.

O seguinte lema dá a medida do conjunto de campos $X\left(f+\mu_{0}+\mu_{1} y+\mu_{3} y^{4}\right)$ que possuem conexões de selas tanto em $C_{0}$ quanto em $C_{ \pm \frac{\pi}{2}}$.

Lembramos a propriedade 3 de $\Sigma^{4}$ : " $X(f) \in \Sigma^{4}$ se

1. $\tilde{X}(f)$ não tem conexão de selas pertencentes a $C_{0}$ ou a $C_{ \pm \frac{\pi}{2}}$,

2. as separatrizes de selas de $\widetilde{X}(f)$ em $C_{0}$ ou em $C_{ \pm \frac{\pi}{2}}$ não são variedades fracas de nós em $C_{ \pm \frac{\pi}{2}}$.'

Lema 3.18 Dados $X(f) \in \mathcal{E}^{4, r}(M)$ com $r \geq 1, \mu_{0} \notin B_{1}^{4}(X(f))$ e $\mu_{3} \notin$ $B_{2}^{4}(X(f))$. Então, o conjunto

$$
\begin{aligned}
B_{6}^{4}\left(X(f) ; \mu_{0}, \mu_{3}\right)=\left\{\mu_{1} \in \Re:\right. & \tilde{X}\left(f+\mu_{0}+\mu_{1} y+\mu_{3} y^{4}\right) \\
& \text { não tem a propriedade } \left.3 \text { de } \Sigma^{4}\right\}
\end{aligned}
$$

tem medida de Lebesgue zero em $\Re$.

Prova. Fixamos $\mu_{0} \notin B_{1}^{4}(X(f))$ e $\mu_{3} \notin B_{2}^{4}(X(f))$.

Como o número de singularidades de $\widetilde{X}\left(f+\mu_{0}+\mu_{3} y^{4}\right)$ é finito, as conexões de selas, também, acontecem em número finito para cada parâmetro $\mu_{1}$.

Basicamente temos três tipos de separatrizes conectando singularidades de $\widetilde{X}(f)$. Todas essas conexões podem ser quebradas com perturbações da forma $\widetilde{X}\left(f+\mu_{0}+\mu_{1} y+\mu_{3} y^{4}\right)$. Na figura 3.5, vemos as possíveis conexões de separatrizes de singularidades.

De fato, a integral de Sotomayor-Melnikov nos permite calcular a derivada da função separação das variedades estáveis e instáveis da seguinte forma

$$
\begin{gathered}
\operatorname{sep}_{\mu_{1}}(0)=-\int_{-\infty}^{+\infty} \exp \left(-\int_{0}^{t} \operatorname{div}\left(\tilde{X}\left(f+\mu_{0}+\mu_{1}^{*} y+\mu_{3} y^{4}\right)\right) d s\right) \\
\cdot \sin ^{2}(v(t)) \cos ^{3}(v(t)) d t .
\end{gathered}
$$

O integrando em (3.6) é uma função não-negativa para $v(t) \in\left[-\frac{\pi}{2}, \frac{\pi}{2}\right]$. Portanto, $\operatorname{sep}_{\mu_{1}}(0)<0$ e as conexões são quebradas sem que possa surgir 


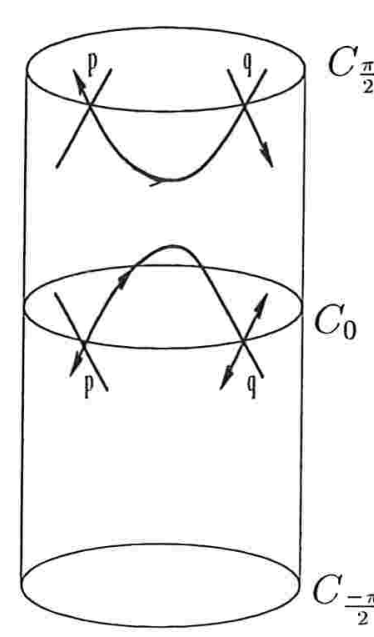

a)

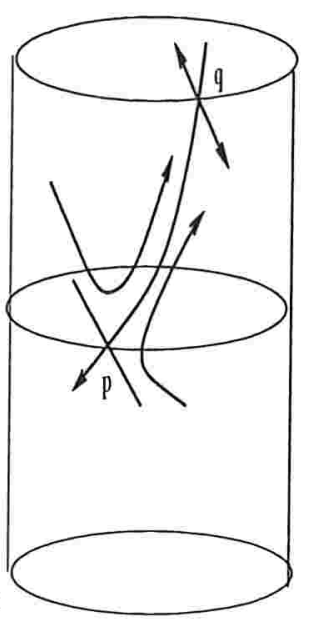

b)

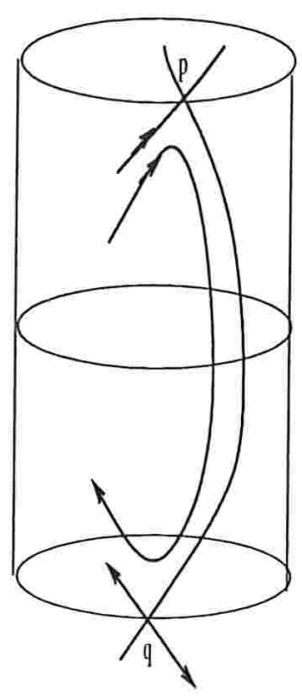

c)

Figura 3.5: Tipos de conexões de separatrizes de singularidades
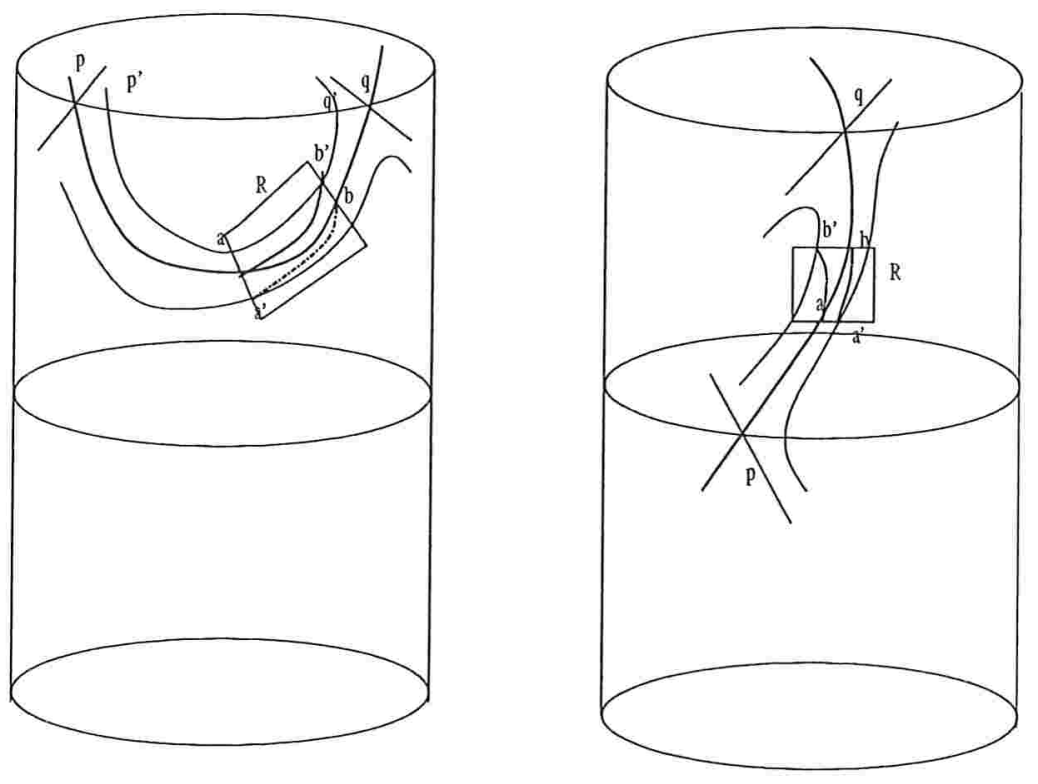

Figura 3.6: Desconexões de separatrizes 
nenhuma outra conexão. Este fato segue de considerar que a perturbação dada pelo parâmetro $\mu_{1}$ sempre aponta na direção y. Ver figura 3.6.

Concluímos que $B_{4}\left(X(f) ; \mu_{0}, \mu_{3}\right)$ é um subconjunto discreto de $\Re$, e portanto de medida de Lebesgue zero.

Prova do Teorema 7. O conjunto $B^{4}(X(f))$ é a união dos seguintes conjuntos:

$$
\begin{gathered}
\mathcal{B}_{1}=B_{1}^{4}(X(f)) \times \Re^{3}, \\
\mathcal{B}_{2}=\Re^{3} \times B_{2}^{4}(X(f)), \\
\mathcal{B}_{3}=\bigcup_{\mu_{0} \in \Re-B_{1}^{4}(X(f))}\left\{\mu_{0}\right\} \times B_{3}^{4}\left(X(f), \mu_{0}\right) \times \Re^{2}, \\
\mathcal{B}_{4}=\Re^{2} \times B_{4}^{4}\left(X(f), \mu_{3}\right) \times \bigcup_{\mu_{3} \in \Re-B_{2}^{4}(X(f))}\left\{\mu_{3}\right\}, \\
\mathcal{B}_{5}=\bigcup_{\mu_{0} \in \Re}\left\{\mu_{0}\right\} \times B_{5}^{4}\left(X(f) ; \mu_{0}\right) \times \Re^{2}
\end{gathered}
$$

$\mathrm{e}$

$$
\mathcal{B}_{6}=\underset{\left(\mu_{0}, \mu_{3}\right) \in\left(\Re-B_{1}^{4}(X(f))\right) \cup\left(\Re-B_{2}^{4}(X(f))\right)}{\bigcup_{0}}\left\{\mu_{0}\right\} \times B_{6}^{4}\left(X(f), \mu_{0}, \mu_{3}\right) \times \Re \times\left\{\mu_{3}\right\},
$$

onde $B_{i}^{4}(X(f), \cdot), i=1, \ldots, 6$, são dados pelos lemas $3.15,3.16,3.17$ e 3.18.

Cada conjunto $\mathcal{B}_{i}$ satisfaz as seguintes condições:

- Contém parâmetros $\left(\mu_{0}, \mu_{1}, \mu_{2}, \mu_{3}\right) \in \Re^{4}$ tais que o campo $X\left(f+\mu_{0}+\mu_{1} y+\mu_{2} y^{3}+\mu_{3} y^{4}\right)$ viola pelo menos uma das propriedades de $\Sigma^{4}$.

- É mensurável. Pois seu complemento em $\Re^{4}$ é um conjunto aberto.

- Tem medida zero em $\Re^{4}$. Pois, $\mathcal{B}_{1}$ e $\mathcal{B}_{2}$ são produtos de $\Re^{3}$ por um conjunto de medida nula em $\Re$. Para calcular a medida de $\mathcal{B}_{i}$ para $i=3, \ldots, 6$, aplicamos o Teorema de Fubini como segue

$$
\int_{\Re^{4}} \chi\left(\mathcal{B}_{2}\right) d \mu_{0} \cdots d \mu_{3}=\int_{\Re^{3}}\left(\int_{\Re} \chi\left(\cdot, \cdot, \cdot, B_{3}^{4}(X(f), \cdot)\right) d \mu_{3}\right) d \mu_{0} d \mu_{1} d \mu_{2}=0
$$




$$
\begin{aligned}
& \int_{\Re^{4}} \chi\left(\mathcal{B}_{3}\right) d \mu_{0} \cdots d \mu_{3}=\int_{\Re^{3}}\left(\int_{\Re} \chi\left(\cdot, B_{4}^{4}(X(f), \cdot), \cdot, \cdot\right) d \mu_{1}\right) d \mu_{0} d \mu_{2} d \mu_{3}=0 \\
& \int_{\Re^{4}} \chi\left(\mathcal{B}_{5}\right) d \mu_{0} \cdots d \mu_{3}=\int_{\Re^{3}}\left(\int_{\Re} \chi\left(\cdot, B_{5}^{4}(X(f), \cdot), \cdot \cdot\right) d \mu_{1}\right) d \mu_{0} d \mu_{2} d \mu_{3}=0 \\
& \int_{\Re^{4}} \chi\left(\mathcal{B}_{6}\right) d \mu_{0} \cdots d \mu_{3}=\int_{\Re^{3}}\left(\int_{\Re} \chi\left(\cdot, B_{6}^{4}(X(f), \cdot), \cdot \cdot\right) d \mu_{1}\right) d \mu_{0} d \mu_{2} d \mu_{3}=0
\end{aligned}
$$

onde $\chi(\cdot)$ é a função indicadora de conjuntos de $\Re^{4}$.

Então, $B^{4}(X(f))$ é união de conjuntos de medida zero em $\Re^{4}$, portanto, é mensurável e com medida de Lebesgue zero em $\Re^{4}$.

Apresentamos a seguir o teorema de genericidade de $\Sigma^{4}$.

Teorema $8 \Sigma^{4}$ é aberto e denso em $\mathcal{E}^{4, r}(M)$ com $r \geq 1$.

Prova. A densidade de $\Sigma^{4}$ segue diretamente do Teorema 7. Pois, para cada $X(f) \in \mathcal{E}^{4, r}$ e $\forall \epsilon>0$ existe $\mu=\left(\mu_{0}, \mu_{1}, \mu_{2}, \mu_{3}\right) \in \Re^{4}$ tal que $\left|X_{\mu}(f)-X(f)\right|<$ $\epsilon$ e $X_{\mu}(f) \in \Sigma^{4}$.

Agora queremos ver que $\Sigma^{4}$ é aberto em $\mathcal{E}^{4, r}$.

Escrevemos $X(f)=y \frac{\partial}{\partial x}+\sum_{i=0}^{4} a_{i}(x) y^{i} \frac{\partial}{\partial y}$ e $X(g)=y \frac{\partial}{\partial x}+\sum_{i=0}^{4} b_{i}(x) y^{i} \frac{\partial}{\partial y}$. Pelas proposições 2.1 e 2.6 , as singularidades de $\tilde{X}(f),\left(u_{*}, \cdot\right)$, estão localizadas no círculo $C_{0}$ satisfazendo as relações $a_{0}\left(u_{*}\right)=0$ e $a_{0}^{\prime}\left(u_{*}\right) \neq 0$, e nos círculos $C_{ \pm \frac{\pi}{2}}$ satisfazendo $a_{4}\left(u_{*}\right)=0$ e $a_{4}^{\prime}\left(u_{*}\right) \neq 0$.

Logo, escolhendo $\mu_{0}, \mu_{1}, \mu_{2}$ e $\mu_{3}$ suficientemente pequeno, o campo $X(g)$ com funções coeficientes $b_{0}=a_{0}+\mu_{0}, b_{1}=a_{1}+\mu_{1}, b_{3}=a_{3}+\mu_{2}$ e $b_{4}=a_{4}+\mu_{3}$ tem singularidades do mesmo tipo que $X(f)$.

Além disso, se $X(f)$ tem uma órbita periódica hiperbólica, o campo $X(g)$ com $b_{1}=a_{1}(u)+\mu_{1}$ para valores pequenos de $\mu_{1}$ terá uma órbita periódica hiperbólica.

\subsection{Caso $n \geq 5$.}

Dado $X(f) \in \mathcal{E}^{n, r}, n \geq 5$ e $r \geq 2$. O campo compactificado $\tilde{X}(f)$ tem singularidades em $C_{0}$ e $C_{ \pm \frac{\pi}{2}}$. 


\subsubsection{Definição de $\Sigma^{n}$}

Definição 3.5 Definimos por $\Sigma^{n}$, para $n \geq 5$, o conjunto dos campos $X(f) \in$ $\mathcal{E}^{n, r}$ com $r \geq 2$ tais que $\tilde{X}(f)$ tem:

1. As singularidades $\left(u_{*}, 0\right) \in C_{0}$ hiperbólicas e as singularidades $\left(u_{*}, \pm \frac{\pi}{2}\right) \in$ $C_{ \pm \frac{\pi}{2}}$ semi-hiperbólicas.

2. As órbitas periódicas hiperbólicas e contidas em $\widetilde{M}$.

3. Nenhuma conexão de separatrizes de singularidades. Mais especificamente

(a) nenhuma conexão de selas pertencentes a $C_{0}$,

(b) separatrizes de selas em $C_{0}$ não são variedades invariantes de singularidades em $C_{ \pm \frac{\pi}{2}}$.

(c) nenhuma conexão entre singularidades pertencentes a $C_{ \pm \frac{\pi}{2}}$ através de variedades invariantes.

\subsubsection{Genericidade de $\Sigma^{n}$}

Teorema 9 Dado $X(f) \in \mathcal{E}^{n, r}(M)$ com $n \geq 5$ e $r \geq 2$, o conjunto

$$
B^{n}(X(f))=\left\{\left(\mu_{0}, \mu_{1}, \mu_{2}\right) \in \Re^{3}: X\left(f+\mu_{0}+\mu_{1} y+\mu_{2} y^{n-1}\right) \notin \Sigma^{n}(M)\right\}
$$

é um subconjunto de medida de Lebesgue zero em $\Re^{3}$.

Primeiro, determinamos a medida do conjunto de campos que têm no mínimo uma singularidade não-hiperbólica em $C_{0}$ ou não semi-hiperbólica em $C_{ \pm \frac{\pi}{2}}$

Lema 3.19 Dado $X(f) \in \mathcal{E}^{n, r}(M)$ para $n \geq 5$ e $r \geq 1$. Então,

a) $O$ conjunto

$$
\begin{array}{r}
B_{1}^{n}(X(f))=\left\{\mu_{0} \in \Re: \widetilde{X}\left(f+\mu_{0}\right)\right. \text { tem alguma singularidade } \\
\left.\left(u_{*}, 0\right) \in C_{0} \text { com } \Delta \widetilde{X}\left(f+\mu_{0}\right)=0\right\}
\end{array}
$$

tem medida de Lebesgue zero em $\Re$.

b) O conjunto

$$
\begin{gathered}
B_{2}^{n}(X(f))=\left\{\mu_{2} \in \Re: \widetilde{X}\left(f+\mu_{2} y^{n-1}\right)\right. \text { tem alguma singularidade em } \\
\left.C_{ \pm \frac{\pi}{2}} \operatorname{com} \sigma \widetilde{X}\left(f+\mu_{2} y^{n-1}\right)=0\right\}
\end{gathered}
$$

tem medida de Lebesgue zero em $\Re$. 
Prova. A prova de $a$ ) é idêntica do Lema 3.15 inciso $a$ ).

b) Para cada singularidade $\left(u, \pm \frac{\pi}{2}\right)$ de $\widetilde{X}\left(f+\mu_{2} y^{n-1}\right)$, a condição $a_{n-1}(u)+$ $\mu_{2}=0$ determina que tal singularidade não é semi-hiperbólica. Porquanto, $B_{2}^{n}(X(f))$ deve ser um conjunto discreto e, assim, de medida nula em $\Re$.

Lema 3.20 Dado $X(f) \in \mathcal{E}^{n, r}(M)$ para $n \geq 5$ e $r \geq 1$, e dado $\mu_{0} \notin$ $B_{1}^{n}(X(f))$. Então, o conjunto

$$
\begin{aligned}
B_{3}^{n}\left(X(f) ; \mu_{0}\right)=\left\{\mu_{1} \in \Re:\right. & \tilde{X}\left(f+\mu_{0}+\mu_{1} y\right) \text { tem alguma } \\
& \text { singularidade não-hiperbólica em } \left.C_{0}\right\}
\end{aligned}
$$

tem medida de Lebesgue zero em $\Re$.

Prova. A prova é idêntica do Lema 3.16 inciso a).

A seguir, apresentamos a medida do conjunto de campos que têm no mínimo uma órbita periódica não-hiperbólica contida no interior de $\widetilde{M}$.

Lema 3.21 Dado $X(f) \in \mathcal{E}^{n, r}(M)$ com $n \geq 5$ e $r \geq 1$. Para $\mu_{0} \in \Re$, o conjunto

$$
\begin{array}{r}
B_{4}^{n}\left(X(f) ; \mu_{0}\right)=\left\{\mu_{1} \in \Re: \tilde{X}\left(f+\mu_{0}+\mu_{1} y\right)\right. \text { tem alguma órbita } \\
\text { periódica não-hiperbólica }\}
\end{array}
$$

tem medida de Lebesgue zero em $\Re$.

Prova. A prova é similar ao Lema 3.3 parte $i)$.

O seguinte lema dá a medida do conjunto de campos $X\left(f+\mu_{0}+\mu_{1} y+\right.$ $\left.\mu_{2} y^{n-1}\right)$ que possuem conexões de separatrizes de singularidades tanto estejam em $C_{0}$ quanto em $C_{ \pm \frac{\pi}{2}}$.

Lembramos a propriedade 3 de $\Sigma^{n} \operatorname{com} n \geq 5$ : " $X(f) \in \Sigma^{n}$ se

1. não tem nenhuma conexão de selas pertencentes a $C_{0}$,

2. as separatrizes de selas em $C_{0}$ não são variedades invariantes de singularidades em $C_{ \pm \frac{\pi}{2}}$.

3. nenhuma conexão entre singularidades pertencentes a $C_{ \pm \frac{\pi}{2}}$ através de variedades invariantes." 
Lema 3.22 Dados $X(f) \in \mathcal{E}^{n}(M)$ com $n \geq 5$ e $r \geq 2$, e dados $\mu_{0} \notin$ $B_{1}^{n}(X(f))$ e $\mu_{2} \notin B_{2}^{n}(X(f))$. Entâo, o conjunto

$$
\begin{aligned}
& B_{5}^{n}\left(X(f) ; \mu_{0}, \mu_{2}\right)=\left\{\mu_{1} \in \Re: \quad \tilde{X}\left(f+\mu_{0}+\mu_{1} y+\mu_{2} y^{n-1}\right)\right. \text { não tem } \\
& \text { a propriedade } \left.3 \text { de } \Sigma^{n}\right\}
\end{aligned}
$$

tem medida de Lebesgue zero em $\Re$.

Prova. A prova é similar ao Lema 3.18. Neste caso, salientamos que a derivada da função separação das variedades estáveis e instáveis, calculada a partir da integral de Sotomayor-Melnikov, é da seguinte forma

$$
\begin{aligned}
\operatorname{sep}_{\mu_{1}}(0)=-\int_{-\infty}^{+\infty} \exp \left(-\int_{0}^{t} \operatorname{div}\left(\tilde{X}\left(f+\mu_{0}+\mu_{1}^{*} y+\mu_{2} y^{n-1}\right)\right) d s\right) \\
\cdot \sin ^{2}(v(t)) \cos ^{n-1}(v(t)) d t .
\end{aligned}
$$

Prova do Teorema 9. O conjunto $B^{n}(X(f))$ é a união dos seguintes conjuntos:

$$
\begin{gathered}
\mathcal{B}_{1}=B_{1}^{n}(X(f)) \times \Re^{2}, \\
\mathcal{B}_{2}=\Re^{2} \times B_{2}^{n}(X(f)), \\
\mathcal{B}_{3}=\bigcup_{\mu_{0} \in \Re-B_{1}^{n}(X(f))}\left\{\mu_{0}\right\} \times B_{3}^{n}\left(X(f), \mu_{0}\right) \times \Re, \\
\mathcal{B}_{4}=\bigcup_{\mu_{0} \in \Re}\left\{\mu_{0}\right\} \times B_{4}^{n}\left(X(f) ; \mu_{0}\right) \times \Re,
\end{gathered}
$$

e

$$
\mathcal{B}_{5}=\bigcup_{\left(\mu_{0}, \mu_{2}\right) \in\left(\Re-B_{1}^{n}(X(f))\right) \cup\left(\Re-B_{2}^{n}(X(f))\right)}\left\{\mu_{0}\right\} \times B_{5}^{n}\left(X(f) ; \mu_{0}, \mu_{2}\right) \times\left\{\mu_{2}\right\}
$$

onde $B_{i}^{n}(X(f), \cdot), i=1, \ldots, 5$, são dados pelos lemas 3.19, 3.20, 3.21 e 3.22 .

Cada conjunto $\mathcal{B}_{i}$ satisfaz as seguintes condições:

- Contém parâmetros $\left(\mu_{0}, \mu_{1}, \mu_{2}\right) \in \Re^{3}$ tais que o campo $X\left(f+\mu_{0}+\mu_{1} y+\mu_{2} y^{n-1}\right)$ viola pelo menos uma das propriedades de $\Sigma^{n}$.

- É mensurável. Pois seu complemento em $\Re^{3}$ é um conjunto aberto. 
- Tem medida zero em $\Re^{3}$. Pois, $\mathcal{B}_{1}$ e $\mathcal{B}_{2}$ são produtos de $\Re^{2}$ por um conjunto de medida nula em $\Re$. Para calcular a medida de $\mathcal{B}_{i}$ com $i=3,4$ e 5, aplicamos o Teorema de Fubini como foi realizado no caso $n=4$ (ver prova do Teorema 7 ).

Então, segue que $B^{n}(X(f))$ é união de conjuntos de medida zero em $\Re^{3}$,e portanto, é mensurável e com medida de Lebesgue zero em $\Re^{3}$.

Apresentamos a seguir o Teorema de genericidade de $\Sigma^{n}$.

Teorema 10 Dado $n \geq 5$. $\Sigma^{n}$ é aberto e denso em $\mathcal{E}^{n, r}(M)$ com $r \geq 2$.

Prova. A densidade de $\Sigma^{n}$ segue diretamente do Teorema 9. Pois, para cada $X(f) \in \mathcal{E}^{n, r}$ e $\forall \epsilon>0$ existe $\mu=\left(\mu_{0}, \mu_{1}, \mu_{2}\right) \in \Re^{3}$ tal que

$\left|X\left(f \mu_{0}+\mu_{1} y+\mu_{2} y^{n-1}\right)-X(f)\right|<\epsilon$ onde $X\left(f \mu_{0}+\mu_{1} y+\mu_{2} y^{n-1}\right) \in \Sigma^{n}$.

Agora, queremos ver que $\Sigma^{n}$ é aberto em $\mathcal{E}^{n, r}$.

Escrevemos $X(f)=y \frac{\partial}{\partial x}+\sum_{i=0}^{n} a_{i}(x) y^{i} \frac{\partial}{\partial y}$ e $X(g)=y \frac{\partial}{\partial x}+\sum_{i=0}^{n} b_{i}(x) y^{i} \frac{\partial}{\partial y}$. Pelas proposições 2.1 e 2.6 , as singularidades de $\widetilde{X}(f),\left(u_{*}, \cdot\right)$, estão localizadas no círculo $C_{0}$ satisfazendo as relações $a_{0}\left(u_{*}\right)=0$ e $a_{0}^{\prime}\left(u_{*}\right) \neq 0$, e nos círculos $C_{ \pm \frac{\pi}{2}}$ satisfazendo $a_{n}\left(u_{*}\right)=0$ e $a_{n-1}\left(u_{*}\right) \neq 0$. Então, escolhendo $\mu_{0}, \mu_{1}$ e $\mu_{2}$ suficientemente pequeno, o campo $X(g)$ com funções coeficientes $b_{0}=a_{0}+\mu_{0}, b_{1}=a_{1}+\mu_{1}$ e $b_{n-1}=a_{n-1}+\mu_{2}$ tem singularidades do mesmo tipo que $X(f)$.

Além disso, se $X(f)$ tem uma órbita periódica hiperbólica, o campo $X(g)$ com $b_{1}=a_{1}(u)+\mu_{1}$ para valores pequenos de $\mu_{1}$ terá uma órbita periódica hiperbólica, também.

\subsection{Caracterização da Estabilidade Estrutu- ral}

Nesta seção provaremos o Teorema de caracterização dos campos $X(f)=$ $y \frac{\partial}{\partial x}+f(x, y) \frac{\partial}{\partial y}$ estruturalmente estáveis onde $f(x, y)=\sum_{i=0}^{n} a_{i}(x) y^{i}, n \geq 1$ e $a_{i}(x)$ são funções periódicas de classe $C^{r}$, com $r \geq 1$ se $n=2,3$, 4, e $r \geq 2$ se $n=1$ ou $n \geq 5$.

Teorema $11 X(f) \in \mathcal{E}^{n, r}$, com $r \geq 1$ se $n=2,3,4$, e $r \geq 2$ se $n=1$ ou $n \geq 5$, é estruturalmente estável se e só se $X(f) \in \Sigma^{n}$.

A prova será dada para todo $n$ e dividida em duas proposições de necessidade e suficiência de $\Sigma^{n}$. Especificaremos o valor de $n$ quando precisarmos destacar as propriedades de $X(f)$. 


\subsubsection{Necessidade}

Proposição 3.1 (Necessidade) Se $X(f) \in \mathcal{E}^{n, r}$ com $r \geq 1$ se $n=2,3,4$ e $r \geq 2$ se $n=1$ ou $\geq 5$ é estruturalmente estável então $X(f) \in \Sigma^{n}$.

Prova. Existe uma vizinhança $\mathcal{U}$ de $X(f)$ em $\mathcal{E}^{n, r}$ tal que para $X(g) \in$ $\mathcal{U}$ existe $h_{g}: \widetilde{M} \rightarrow \widetilde{M}$ homeomorfismo que leva trajetórias de $\widetilde{X}(f)$ em trajetórias de $\widetilde{X}(g)$.

Pela densidade de $\Sigma^{n}$, existe um campo $X\left(\sum_{i=0}^{n} b_{i}(x) y^{i}\right) \in \Sigma^{n} \cap \mathcal{U}$ tal que $X(f)$ é topologicamente equivalente a $X\left(\sum_{i=0}^{n} b_{i}(x) y^{i}\right)$. Então, $X(f)$ herda as seguintes propriedades das trajetórias de $X\left(\sum_{i=0}^{n} b_{i}(x) y^{i}\right)$ :

- Em $C_{0}, X(f)$ tem um número finito de singularidades, todas topologicamente equivalentes a selas ou anti-selas.

- Em $C_{ \pm \frac{\pi}{2}}, X(f)$ tem um número finito de tangências (quando $n=3$ ) ou de singularidades (quando $n \geq 4$ ). As tangências são localmente internas ou externas.

- As órbitas periódicas formam um conjunto finito, todas são atratoras ou repulsoras e contidas no interior de $\widetilde{M}$.

- Não tem conexões de separatrizes de singularidades, nem separatrizes de selas tangentes a $C_{ \pm \frac{\pi}{2}}$.

Resta provar as seguintes propriedades de $X(f)$.

a) As singularidades em $C_{0}$ são todas hiperbólicas. De fato, se $\Delta \widetilde{X}(f)=$ 0 em alguma singularidade, digamos $\left(u_{*}, 0\right)$, podemos considerar o campo $\widetilde{X}\left(f+\epsilon \beta(u)\left(u-u_{*}\right)\right)$ onde $\beta$ é uma função periódica, nãonegativa em uma vizinhança $V$ de $u_{*}$ e com suporte compacto contido em $V, \beta\left(u_{*}\right)=1$ e $V$ não contém nenhuma outra singularidade. Segue que $\Delta \widetilde{X}(f)\left(u_{*}, \cdot\right)=-\epsilon \beta\left(u_{*}\right)$. Então, escolhendo o sinal apropriado para $\epsilon$, obtemos que $\left(u_{*}, \cdot\right)$ sendo também uma singularidade de $\widetilde{X}\left(f+\epsilon \beta(u)\left(u-u_{*}\right)\right)$ tem índice distinto como singularidade de $\widetilde{X}(f)$, contradizendo a estabilidade estrutural de $X(f)$.

b) Em $C_{ \pm \frac{\pi}{2}}$, segundo o valor de $n$, temos singularidades hiperbólicas, semihiperbólicas, ou pontos de tangências quadráticas.

Para $n=3$, suponhamos que $\left(u_{*}, \frac{\pi}{2}\right)$ é um ponto de tangência nãoquadrática, mas é localmente interna ou externa. Isto é, $\left(u_{*}, \frac{\pi}{2}\right)$ satisfaz $a_{3}\left(u_{*}\right)=0$ e $a_{3}^{\prime}\left(u_{*}\right)=0$. 
Seja $V$ uma vizinhança de $u_{*}$ em $\Re$ tal que não contenha nenhum outro ponto de tangência de $\widetilde{X}(f)$. O campo $X\left(f+\epsilon\left(x-u_{*}\right) \beta(x) y^{3}\right)$ onde $\beta(x)$ é uma função periódica não-negativa de classe $C^{r}$ com suporte contido em $V$, está suficientemente próximo de $X(f)$ conforme $\epsilon$ seja pequeno. Vemos que $\left(u_{*}, \frac{\pi}{2}\right)$ é um ponto de tangência de $X\left(f+\epsilon\left(x-u_{*}\right) \beta(x) y^{3}\right)$ com $\partial \widetilde{M}$, pois

$$
X\left(f+\epsilon\left(x-u_{*}\right) \beta(x) y^{3}\right) \cdot b\left(u_{*}, \frac{\pi}{2}\right)=0
$$

e mais ainda, a tangência é quadrática, pois

$$
X^{2}\left(f+\epsilon\left(x-u_{*}\right) \beta(x) y^{3}\right) \cdot b\left(u_{*}, \frac{\pi}{2}\right)=\epsilon \neq 0
$$

onde $b: \widetilde{M} \rightarrow \Re$ é definida por $b(u, v)=\cos (v)$ como foi introduzida na Proposição 2.5 .

Então, se $\left(u_{*}, \frac{\pi}{2}\right)$ é um ponto de tangência externa (respectivamente, interna) de $\widetilde{X}(f)$, tomamos um $\epsilon$ positivo (respectivamente, negativo) com tal que $X\left(f+\epsilon\left(x-u_{*}\right) \beta(x) y^{3}\right)$ tenha em $\left(u_{*}, \frac{\pi}{2}\right)$ um ponto de tangência interna (respectivamente, externa), contradizendo a estabilidade estrutural de $X(f)$.

Para $n=4$, a hiperbolicidade de todas as singularidades de $\tilde{X}(f)$ em $C_{ \pm \frac{\pi}{2}}$ pode ser mostrada da mesma maneira que o inciso $a$ ), considerando os sistemas próximo a $X(f)$ da forma $X\left(f+\epsilon\left(x-u_{*}\right) \beta(x) y^{4}\right)$ onde $\left(u_{*}, \pm \frac{\pi}{2}\right)$ é uma singularidade de $\widetilde{X}(f)$.

Só precisamos tratar aqui, o caso em que uma singularidade de $\widetilde{X}(f)$ tenha associado autovalores de $D \tilde{X}(f)$ iguais. De fato, se $\left(u_{*}, \pm \frac{\pi}{2}\right)$ é uma tal singularidade de $\tilde{X}(f)$, então satisfaz as seguintes condições: $a_{4}^{\prime}\left(u_{*}\right)>0$ e $a_{3}\left(u_{*}\right)=2 \sqrt{a_{4}^{\prime}\left(u_{*}\right)}$. Logo, tomando um campo suficientemente próximo a $\widetilde{X}(f)$ da forma $X\left(f+\epsilon\left(x-u_{*}\right) \beta(x) y^{3}\right)$ onde $\beta(x)$ é uma função periódica não-negativa com suporte contido em uma vizinhança de $u_{*}$ e sem conter nenhuma outra singularidade, obtemos que $\left(u_{*}, \pm \frac{\pi}{2}\right)$ é, também, uma singularidade de $X\left(f+\epsilon\left(x-u_{*}\right) \beta(x) y^{3}\right)$, mas com autovalores associados não iguais. Mais especificamente, se $\epsilon<2 \sqrt{a_{4}^{\prime}\left(u_{*}\right)}-$ $a_{3}\left(u_{*}\right)$, a singularidade é um foco, e se $\epsilon>2 \sqrt{a_{4}^{\prime}\left(u_{*}\right)}-a_{3}\left(u_{*}\right)$ a singularidade é um nó hiperbólico. Portanto, os campos $X\left(f+\epsilon\left(x-u_{*}\right) \beta(x) y^{3}\right)$ e $X(f)$ não podem ser topologicamente equivalentes.

Para $n \geq 5$, se $\left(u_{*}, \pm \frac{\pi}{2}\right)$ é uma singularidade de $\tilde{X}(f)$ não semihiperbólica, então, satisfaz as condições: $a_{n}\left(u_{*}\right)=0$ e $a_{n-1}\left(u_{*}\right)=0$. 
Agora, o campo suficientemente próximo a $\widetilde{X}(f)$ da forma $X(f+\epsilon(x-$ $\left.\left.u_{*}\right) \beta(x) y^{n-1}\right)$, tem a $\left(u_{*}, \pm \frac{\pi}{2}\right)$ como uma singularidade semi-hiperbólica. Isto contradiz a estabilidade estrutural de $X(f)$.

c) As órbitas periódicas são todas hiperbólicas. De fato, suponhamos que $\gamma(s)=(\varphi(s), \xi(s))$ seja uma órbita periódica de período $\tau$ de $X(f)$ tal que seu índice característico $\chi(\gamma, X(f))=0$. Conseguiremos um campo $X(f+g)$ suficientemente próximo de $X(f)$ tendo a $\gamma$ como órbita periódica, mas com índice $\chi(\gamma, X(f+g)) \neq 0$.

Procuramos, então, uma função $g(x, y)=b_{0}(x)+b_{1}(x) y$ com $b_{0}$ e $b_{1}$ periódicas de classe $C^{r}$ tal que

i) $X(f+g)$ esteja próximo de $X(f)$ digamos a menos de $\epsilon \neq 0$.

ii) $X(f+g)(\gamma)=X(f)(\gamma)$ (i.e. $\gamma$ é uma órbita periódica de ambos campos).

iii) $\chi(\gamma, X(f+g)) \neq 0$

As condições $i), i i), i i i)$ anteriores determinam as seguintes:

$* \sup \left|b_{i}^{(k)}(x)\right|<\epsilon$ para $i, k=0, \ldots, r$ e $x \in S^{1}$.

* $b_{0}(\varphi(s))+b_{1}(\varphi(s)) \xi(s)=0$

* $b_{1}(\varphi(s))$ não é identicamente nula em $\Re$.

O problema se reduz, então, a encontrar uma função $b_{1}: \Re \rightarrow \Re$ de classe $C^{r}$ periódica e que não seja identicamente nula. Em seguida, com tal $b_{1}(u)$, podemos escolher $b_{0}: \Re \rightarrow \Re$ de classe $C^{r}$ periódica e que satisfaz $b_{0}(\varphi(s))=-b_{1}(\varphi(s)) \xi(s)$ para $s \in \Re$.

Devemos ter em conta as três situações seguintes.

1) A função $b_{0}$ não pode ser nula. Pois, caso contrário, teríamos que $\xi(s)=0$ para $s \in \Re$ e portanto o círculo $C_{0}$ seria uma órbita periódica de $X(f)$, contradizendo o fato que toda órbita periódica só pode interceptar $C_{0}$ em pontos isolados.

2) Se $\xi(s)$ for constante não-nula $\xi_{0}$, então podemos pôr $b_{1}(x)=\epsilon$ constante não-nula e $b_{0}(x)=-\epsilon \xi_{0}$ constante, também. Logo, vemos que a função $g(x, y)=-\epsilon \xi_{0}+\epsilon y \operatorname{com} \epsilon \neq 0$ pequeno, permite definir uma perturbação $|\epsilon|$-próxima de $X(f)$ e com $\chi(\gamma, X(f+g))=\epsilon$.

3) No caso que $\xi(s)$ não for constante, podemos escolher algum $s_{0} \in$ $(0, \tau)$ tal que $\xi\left(s_{0}\right) \neq 0$ e $\varphi\left(s_{0}\right) \neq 0$. Seja $W=\varphi([0, \tau]) \subset \Re$. Então, existe $U$, vizinhança aberta de $s_{0}$ contida em $[0, \tau]$, tal que $\varphi_{\mid U}$ tem inversa diferenciável (pois $\varphi^{\prime}\left(s_{0}\right)=\xi\left(s_{0}\right) \neq 0$ ). 
Suponhamos que $\xi\left(s_{0}\right)>0$. Denotamos $\widetilde{U}=\varphi(U) \subset W$ e seja $V=$ $(\alpha, \beta)$ uma vizinhança de $\varphi\left(s_{0}\right)$ tal que $V \subset \bar{V} \subset \widetilde{U}$. (Ver figura 3.7).

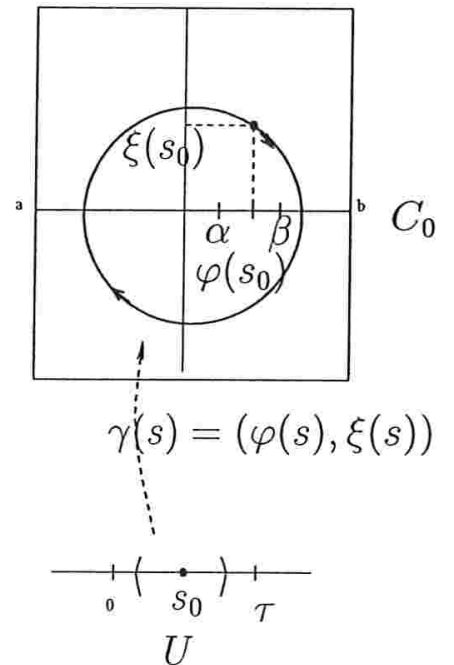

a)

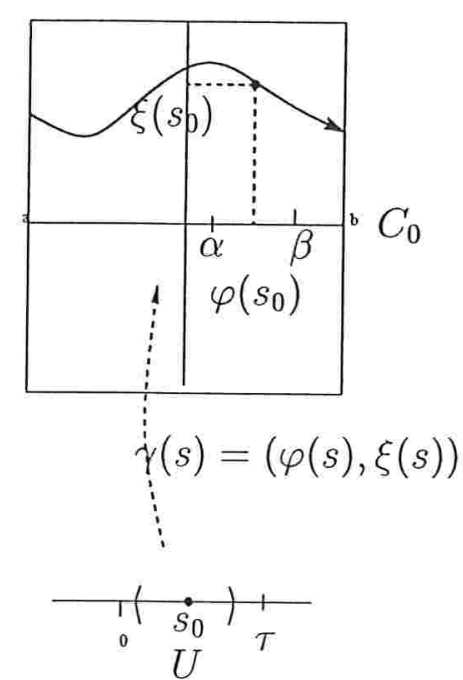

b)

Figura 3.7: a) Órbita periódica homotópica a zero. b) Órbita periódica circundando o cilindro. Os pontos $a$ e $b$ são identificados

Agora, podemos tomar uma função $b_{1}: \Re \rightarrow \Re$ com as seguintes propriedades:

* $b_{1}(x)>0$ para $x \in V$ e $b_{1}(x)=0$ para $x \in W-V$

* classe $C^{r}$, com $r \geq 1$

* estendida periodicamente a todo $\Re$.

Logo, $b_{0}: \Re \rightarrow \Re$ se define como $b_{0}(x)=-b_{1}(x) \varphi^{\prime}\left(\varphi^{-1}(x)\right)$ para $x \in$ $V$ e $b_{0}=0$ para $x \in W-V$. Essa função $b_{0}$ pode ser estendida periodicamente a todo $\Re$ e é de classe $C^{r}$.

Segue que a função $g(x, y)=\epsilon b_{0}(x)+\epsilon b_{1}(x) y$ é uma perturbação de $f(x, y)$ procurada. De fato, o campo $X(f+g)$ tem a $\gamma$ como uma órbita periódica com índice característico

$$
\chi(\gamma, X(f+g))=\epsilon \int_{\varphi^{-1}(\alpha)}^{\varphi^{-1}(\beta)} b_{1}(\varphi(s)) d s \neq 0 .
$$

Se $\gamma$ for atratora para $X(f)$, então tomando $\epsilon>0$, o campo $X(f+g)$ terá pelo menos duas órbitas periódicas a mais que $X(f)$, contradizendo 
o fato de $X(f)$ ser estruturalmente estável. Isto conclui a prova do caso c).

Portanto, os casos $a$ ), b) e c) junto com as propriedades mencionadas acima mostram que $X(f) \in \Sigma^{n}$.

\subsubsection{Suficiência}

Nesta seção, provamos que as propriedades de $\Sigma^{n}$ são suficientes para a estabilidade estrutural. Para isso, dividiremos $\widetilde{M}$ em regiões canônicas e construiremos um homeomorfismo sobre cada uma delas.

Denotamos por $W$ o conjunto de todas as separatrizes de $\widetilde{X}(f)$ em $\widetilde{M}$. (Ver definição 1.4)

Definição 3.6 Uma região canônica de $\widetilde{X}(f)$ é uma componente conexa de $\widetilde{M}-W$.

Definição $3.7 U m$ atrator (resp. uma fonte) de $\widetilde{X}$ associado a uma região canônica $R \subset \widetilde{M}$ é um nó, um foco ou ciclo limite de $X$ ou é um arco da fronteira de $R$ aonde as trajetórias contidas em $R$ tendem quando $t \rightarrow+\infty$ (resp. $t \rightarrow-\infty)$.

Definição 3.8 a) Uma região crítica de um ponto singular $p$ de $\tilde{X}(f)$ é uma vizinhança $D$ de $p$ tal que os sistemas suficientemente próximos a $\tilde{X}$ têm uma única singularidade e do mesmo tipo que $p$.

b) Uma região crítica de um ciclo limite $\Gamma$ de $\widetilde{X}(f)$ é um anel $A$ contendo $\Gamma$ tal que os sistemas suficientemente próximos $\widetilde{X}(f)$ têm um único ciclo limite e da mesma estabilidade que $\Gamma$.

Toda região canônica tem na sua fronteira somente uma fonte e um atrator. Esta propriedade, de grande utilidade para a classificação das regiões canônicas, foi dada em [18].

Denotamos por $\alpha, \omega$ e $\gamma$ uma fonte, um atrator e uma separatriz de $\widetilde{X}$ respectivamente. Para os casos em que uma separatriz seja um ponto (i.e. uma singularidade ou uma tangência externa) usamos $p$.

Classificamos uma região canônica $R$ pelo número e tipo de separatrizes contidas em sua fronteira $\partial R$.

- Tipo i. $\partial R=\{\alpha, \omega\}$. Por exemplo, uma região limitada por dois ciclos limites sem singularidades em seu interior. 
- Tipo ii. $\partial R=\left\{\alpha, \omega, \gamma_{1}\right\}$ onde $\gamma_{1}$ é uma trajetória transversal a $\partial M$ e $\alpha$ e $\omega$ pertencem a $\partial M$.

- Tipo iii. $\partial R=\left\{\alpha, \omega, \gamma_{1}, p_{1}\right\}$ onde $\gamma_{1}$ é uma trajetória com tangência interna a $\partial M$ e $p_{1}$ é um ponto de tangência externa.

- Tipo iv. $\partial R=\left\{\alpha, \omega, \gamma_{1}, \gamma_{2}\right\}$ onde $\gamma_{1}$ e $\gamma_{2}$ são trajetórias separatrizes.

- Tipo v. $\partial R=\left\{\alpha, \omega, p_{1}, \gamma_{1}, \gamma_{2}\right\}$ onde $p_{1}$ é uma sela, $\gamma_{1}$ e $\gamma_{2}$ são trajetórias que tendem a $p_{1}$.

- Tipo vi. $\partial R=\left\{\alpha, \omega, p_{1}, \gamma_{1}, \gamma_{2}, \gamma_{3}\right\}$ onde $p_{1}$ é uma sela, $\gamma_{1}, \gamma_{2}$ são trajetórias que tendem a $p_{1}$ e $\gamma_{3}$ é uma trajetória que não tende a $p_{1}$.

- Tipo vii. $\partial R=\left\{\alpha, \omega, p_{1}, \gamma_{1}, \gamma_{2}, \gamma_{3}\right\}$ onde $p_{1}$ é uma sela, $\gamma_{1}, \gamma_{2}$ e $\gamma_{3}$ são trajetórias que tendem a $p_{1}$.

- Tipo viii. $\partial R=\left\{\alpha, \omega, p_{1}, \gamma_{1}, \gamma_{2}, p_{2}, \gamma_{3}\right\}$ onde $p_{1}$ e $p_{2}$ são selas, $\gamma_{1}, \gamma_{2}$ trajetórias que tendem a $p_{1}$ e $\gamma_{3}$ trajetória que tende a $p_{2}$.

- Tipo ix. $\partial R=\left\{\alpha, \omega, p_{1}, \gamma_{1}, \gamma_{2}, p_{2}, \gamma_{3}, \gamma_{4}\right\}$ onde $p_{1}$ e $p_{2}$ são selas, $\gamma_{1}, \gamma_{2}$ trajetórias que tendem a $p_{1}$ e $\gamma_{3}$ e $\gamma_{4}$ trajetórias que tendem a $p_{2}$.

Na figura 3.8, apresentamos os tipos de regiões canônicas que encontramos em $\widetilde{M}$.

Em [18], Peixoto e Peixoto apresentaram cinco tipos de regiões canônicas. Posteriormente, Sotomayor em [19] estendeu o número de regiões a sete, considerando as tangências externas e internas entre a fronteira de $\widetilde{M}$ e as trajetórias que não são separatrizes de selas. Em [22], Teixeira apresentou outras regiões canônicas ao estudar as bifurcações genéricas sobre variedades com fronteira. Em [16], Neumann classificou os fluxos contínuos sobre variedades bidimensionais pela equivalência topológica das regiôes canônicas que os fluxos determinam.

Na tabela 3.1, mostramos as classificações de Peixoto-Peixoto e de Sotomayor em função da classificação dada neste trabalho.

Observar que os tipos $i i, v$ e viii são novos. Estes surgem pela presença de singularidades na fronteira de $\widetilde{M}{ }^{1}$

\footnotetext{
${ }^{1}$ A região canônica do tipo $i i$ foi observada e discutida pelo Prof. Ronaldo Garcia na fase final da tese.
} 


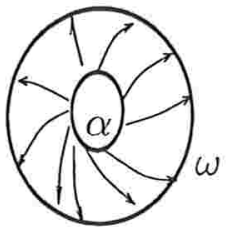

Tipo i.

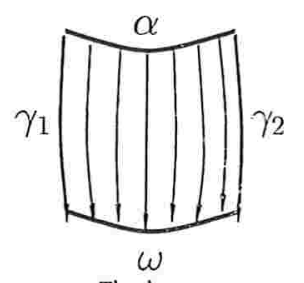

Tipoiv.

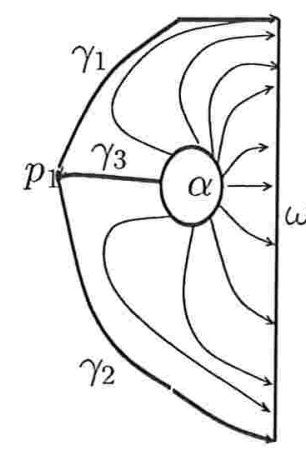

Tipo vii.

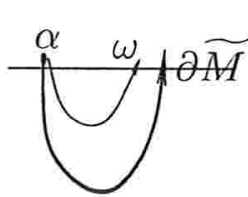

Tipo ï.

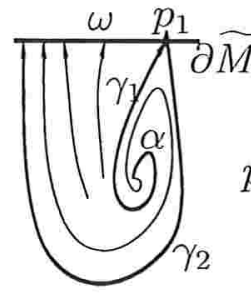

Tipov.

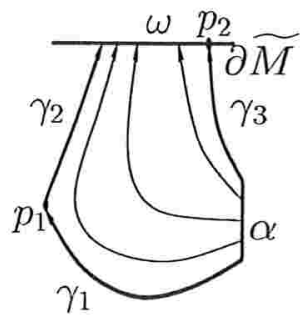

Tipo viii.

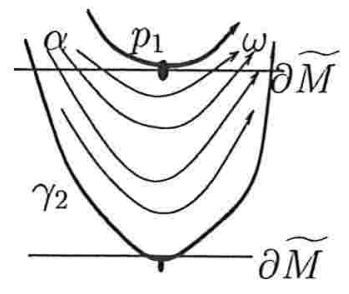

Tipo iii.

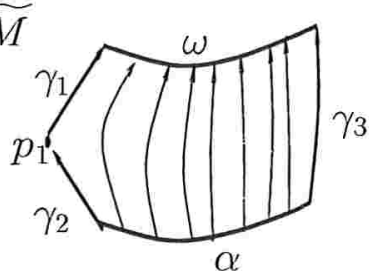

Tipo vi.

Figura 3.8: Regiões canônicas classificadas segundo o número e tipo de separatrizes que formam a fronteira. 


\begin{tabular}{llcc} 
& Tipo & Peixoto-Peixoto & Sotomayor \\
\hline i. & $\partial R=\{\alpha, \omega\}$ & I & 1 \\
ii. & $\partial R=\left\{\alpha, \omega, \gamma_{1}\right\}$ & & 6 \\
iii. & $\partial R=\left\{\alpha, \omega, \gamma_{1}, p_{1}\right\}$ & & 7 \\
iv. & $\partial R=\left\{\alpha, \omega, \gamma_{1}, \gamma_{2}\right\}$ & II & \\
v. & $\partial R=\left\{\alpha, \omega, p_{1}, \gamma_{1}, \gamma_{2}\right\}$ & & 4,5 \\
vi. & $\partial R=\left\{\alpha, \omega, p_{1}, \gamma_{1}, \gamma_{2}, \gamma_{3}\right\}$ & III & 3 \\
vii. & $\partial R=\left\{\alpha, \omega, p_{1}, \gamma_{1}, \gamma_{2}, \gamma_{3}\right\}$ & IV & \\
viii. & $\partial R=\left\{\alpha, \omega, p_{1}, \gamma_{1}, \gamma_{2}, p_{2}, \gamma_{3}\right\}$ & & 2 \\
ix. & $\partial R=\left\{\alpha, \omega, p_{1}, \gamma_{1}, \gamma_{2}, p_{2}, \gamma_{3}, \gamma_{4}\right\}$ & V &
\end{tabular}

Tabela 3.1: Classificação das regiões canônicas segundo Peixoto-Peixoto (I$\mathrm{V})$, Sotomayor $(1, \ldots, 7)$ e deste trabalho $(i, i i, \ldots, i x)$.

\section{Teorema da suficiência}

Nesta seção usaremos as seguintes notações:

1. $A B$ indicará o arco que une os pontos $A$ e $B$.

2. $l(A B)$ indicará o comprimento de arco.

3. A função $Z_{A B}: A B \rightarrow[0,1]$ definida por $Z_{A B}(M)=\frac{l(A M)}{l(A B)}$ para $M \in$ $A B$, é chamada de razão de comprimento de arco. Denotaremos por $Z_{A B}^{-1}$ a inversa de $Z_{A B}$.

Teorema 12 Para cada $Y(g) \in \mathcal{E}^{n, r}$ (com $r \geq 2$ se $n=1$ ou $n \geq 5$, e $r \geq 1$ se $n=2,3,4)$ suficientemente próximo de $X(f) \in \Sigma^{n}$, existe um homeomorfismo $T: \widetilde{M} \rightarrow \widetilde{M}$ que leva trajetórias de $\widetilde{X}(f)$ em trajetórias de $\tilde{Y}(g)$.

A prova deste teorema seguirá de vários lemas.

Lema 3.23 [Invariância topológica das regiões canônicas] Dado $X(f) \in \Sigma^{n}$ sobre $M=S^{1} \times \Re$. Seja $R$ uma região canônica de $\tilde{X}(f)$. Então, para cada $Y(g) \in \mathcal{E}^{n, r}$ suficientemente próximo de $X(f)$, existe um homeomorfismo $T_{R}: R \rightarrow \widetilde{R}$ entre as regiões canônicas $R$ de $\widetilde{X}(f)$ e $\widetilde{R}$ de $\widetilde{Y}(g)$ tal que

1. se $S \in \partial R$ é uma separatriz de $\widetilde{X}(f)$ entãa $T_{R}(S)$ é uma separatriz de $\widetilde{Y}(g)$ do mesmo tipo que $S$ e $T_{R}(S) \in \partial \widetilde{R}$ 
2. $T_{R}(\partial R)=\partial \widetilde{R}$

3. leva trajetórias de $\widetilde{X}(f)$ em trajetórias de $\widetilde{Y}(g)$.

Prova. Para cada tipo de região canônica, construímos um homeomorfismo por razão de comprimento de arco. Apresentamos detalhes das regiões dos tipos $v$ e $i x$.

- Região Tipo $i x$. A fronteira de $R$ (respectivamente $\widetilde{R}$ ) é descrita pelas selas $K_{0}$ e $K_{1}$ (resp. $\widetilde{K}_{0}$ e $\widetilde{K}_{1}$ ), pelos arcos de separatrizes $A_{i} K_{i}$ e $K_{i} B_{i}$ (resp. $\widetilde{A}_{i} \widetilde{K}_{i}$ e $\widetilde{K}_{i} \widetilde{B}_{i}$ ) para $i=0,1$ e pelos $\operatorname{arcos} A_{0} A_{1}$ e $B_{0} B_{1}$ (resp. $\widetilde{A}_{0} \widetilde{A}_{1}$ e $\widetilde{B}_{0} \widetilde{B}_{1}$ ).

Definimos a função $\varphi: R \rightarrow \widetilde{R}$ como segue:

Sobre a fronteira de $R$, os arcos de separatrizes de selas são aplicados nos correspondentes de $\widetilde{R}$ da seguinte forma:

$$
\varphi(A)= \begin{cases}Z_{\widetilde{A}_{0} \widetilde{A}_{1}}^{-1} \circ Z_{A_{0} A_{1}}(A) & \text { se } A \in A_{0} A_{1} \\ Z_{\widetilde{B}_{0} \widetilde{B}_{1}}^{-1} \circ Z_{B_{0} B_{1}}(A) & \text { se } A \in B_{0} B_{1} \\ Z_{\widetilde{A}_{i} \widetilde{K}_{i}}^{-1} \circ Z_{A_{i} K_{i}}(A) & \text { se } A \in A_{i} K_{i}, i=0,1 \\ Z_{\widetilde{K}_{i} \widetilde{B}_{i}}^{-1} \circ Z_{K_{i} B_{i}}(A) & \text { se } A \in K_{i} B_{i}, i=0,1\end{cases}
$$

Estendemos $\varphi$ para o interior de $R$ da seguinte maneira. Para cada trajetória $A B$ com $A \in A_{0} A_{1}$ e $B \in B_{0} B_{1}$, consideramos um $M \in A B$ tal que

$$
Z_{A B}(M)=\left(1-Z_{A_{0} A_{1}}(A)\right) Z_{A_{0} K_{0} B_{0}}\left(K_{0}\right)+Z_{A_{0} A_{1}}(A) Z_{A_{1} K_{1} B_{1}}\left(K_{1}\right) .
$$

O conjunto dos $M$ que satisfazem 3.8 constitui uma curva $\Gamma$ que une as selas $K_{0}$ e $K_{1}$.

Para $P \in \operatorname{int}(R)$, existe uma trajetória $A B$ que passa por $P$. Então, definimos $\varphi$ em $P$ como segue

$$
\varphi(P)= \begin{cases}Z_{\widetilde{A} \widetilde{M}}^{-1} \circ Z_{A M}(P) & \text { se } P \in A M \text { onde } M \in \Gamma(A) \\ Z_{\widetilde{M} \widetilde{B}}^{-1} \circ Z_{M B}(P) & \text { se } P \in M B \text { e } M \in \Gamma(A)\end{cases}
$$


onde $\widetilde{M}$ satisfaz

$$
Z_{\widetilde{A} \widetilde{B}}(\widetilde{M})=Z_{\widetilde{A}_{0} \widetilde{K}_{0} \widetilde{B}_{0}}\left(\widetilde{K}_{0}\right)+Z_{\widetilde{A}_{0} \widetilde{A}_{1}}(\widetilde{A})\left(Z_{\widetilde{A}_{1} \widetilde{K}_{1} \widetilde{B}_{1}}\left(\widetilde{K}_{1}\right)-\left(Z_{\widetilde{A}_{0} \widetilde{K}_{0} \widetilde{B}_{0}}\left(\widetilde{K}_{0}\right)\right)\right.
$$

A função $\varphi: R \rightarrow \widetilde{R}$, assim definida, é $1-1$ e sobrejetiva. A função inversa $\varphi^{-1}$ é definida da mesma maneira que $\varphi$. A continuidade de $\varphi$ para todo $P \in R-\left\{A_{i} K_{i} B_{i}\right\}$ com $i=0,1$, segue da continuidade das soluções com respeito aos valores iniciais.

- Região Tipo $v$. A fronteira de $R$ (respectivamente $\widetilde{R}$ ) é descrita pela singularidade $K$ (resp. $\widetilde{K})$, pelas trajetórias $K A_{0}\left(\operatorname{resp} . \widetilde{K} \widetilde{A}_{0}\right)$ e $B_{0} K$ $\left(\operatorname{resp} . \widetilde{B}_{0} \widetilde{K}\right)$ e pelos $\operatorname{arcos} B_{0} B_{0}$ e $A_{0} K\left(\operatorname{resp} . \widetilde{B}_{0} \widetilde{B}_{0}\right.$ e $\left.\widetilde{A}_{0} \widetilde{K}\right)$.

Um arco de fronteira de $R$ é levado ao correspondente arco de fronteira de $\widetilde{R}$ por razão de comprimento de arco da seguinte maneira:

$$
\varphi(A)=\left\{\begin{array}{cc}
Z_{\widetilde{A}_{0} \widetilde{K}}^{-1} \circ Z_{A_{0} K}(A) & \text { se } A \in A_{0} K \\
Z_{\widetilde{K} \widetilde{A}_{0}}^{-1} \circ Z_{K A_{0}}(A) & \text { se } A \in K A_{0} \\
Z_{\widetilde{B}_{0} \widetilde{K}}^{-1} \circ Z_{B_{0} K}(A) & \text { se } A \in B_{0} K
\end{array}\right.
$$

Estendemos $\varphi$ para o interior de $R$ da seguinte maneira.

Para $P \in \operatorname{int}(R)$, existe uma trajetória $B A$ que passa por $P$ onde $B \in B_{0} B_{0}$ e $A \in A_{0} K$. Então, definimos $\varphi$ em $P$ como segue

$$
\varphi(P)=Z_{\widetilde{B} \tilde{A}}^{-1} \circ Z_{B A}(P) .
$$

A função $\varphi: R \rightarrow \widetilde{R}$, assim definida, é $1-1$ e sobrejetiva. A função inversa $\varphi^{-1}$ é definida da mesma maneira que $\varphi$. A continuidade de $\varphi$, segue da continuidade das soluções com respeito aos valores iniciais.

Lema 3.24 Dado $X(f) \in \Sigma^{n}$ sobre $M=S^{1} \times \Re$. Para uma região crítica $C$ associada a uma singularidade de $\widetilde{X}(f)$ pertencente a $\widetilde{M}$ ou a um ponto de tangência de $\tilde{X}(f)$ com $\partial \widetilde{M}$ ou a um ciclo limite de $\widetilde{X}(f)$, existe um homeomorfismo $T_{C}: C \rightarrow C$ que leva trajetórias de $\widetilde{X}(f)$ em trajetórias de $\widetilde{Y}(g) \in \mathcal{E}^{n, r}$ suficientemente próximo de $X(f)$. 
Prova. As regiões críticas são discos para as singularidades de $\widetilde{X}(f)$ contidas no interior de $\widetilde{M}$, anéis para os ciclos limites de $\widetilde{X}(f)$ e arcos de círculos para as singularidades e para os pontos de tangências de $\widetilde{X}(f)$ na fronteira de $\widetilde{M}$.

A construção de um homeomorfismo para cada região crítica, foi realizada em $[19,18]$ aplicando a razão de comprimento de arco ou indiferentemente uma parametrização pelo tempo. Explicitamos, aqui, essa construção para as regiões críticas de uma singularidade atratora e de um ciclo limite atrator.

1. Seja $D$ uma região crítica de $K$, uma singularidade atratora de $\widetilde{X}(f)$. Para $\widetilde{X}(g)$ suficientemente próximo de $\widetilde{X}(f)$, existe um único $\widetilde{K}$ atrator contido em $D$.

Como $\partial D$ está contida em regiões canônicas, temos definido um homeomorfismo $T_{\partial D}: \partial D \rightarrow \partial D$ por razão de comprimento de arco.

Definimos a função $\varphi: D \rightarrow D$ como segue:

$$
\varphi(K)=\widetilde{K} .
$$

para $M \in D$, existem $v \in \partial D$ e $t \in \Re$ tais que a trajetória $\gamma_{v}$ de $\widetilde{X}(f)$ que passa por $v$ satisfaz $M=\gamma_{v}(t)$. Então, $\varphi(M)$ é definido por $\widetilde{\gamma}_{\widetilde{v}}(t)$ onde $\widetilde{v}=T_{\partial D}(v)$. Esta função é um homeomorfismo.

Outra forma de definir $\varphi$ é por razão de comprimento de arco da seguinte maneira:

$$
\varphi(M)= \begin{cases}T_{\partial D}(M) & \text { se } M \in \partial D \\ Z_{\widetilde{v} \widetilde{K}}^{-1} \circ Z_{v K}(M) & \text { se } M \in D^{\circ}-K \\ \widetilde{K} & \text { se } M=K\end{cases}
$$

onde $v K$ e $\widetilde{v} \widetilde{K}$ são arcos de trajetórias de $\widetilde{X}(f)$ e $\widetilde{X}(g)$ respectivamente. Pela continuidade das soluções de $\widetilde{(} X)(f)$ com respeito aos valores iniciais, $\varphi$ em $D-\{K\}$. Da mesma maneira, podemos definir $\varphi^{-1}$. Portanto, $\varphi$ é contínua em todo $D$.

2. Sejam $\Gamma$ e $\widetilde{\Gamma}$ os ciclos limites de $\widetilde{X}(f)$ e $\widetilde{X}(g)$ respectivamente, contidos em uma região crítica $S$ (anel).

Sejam $C$ e $C^{\prime}$ as curvas fronteira de $S$. Essas curvas são enviadas homeomorficamente sobre si mesmas por razão de comprimento de arco. Chamamos esses homeomorfismo por $T_{C}$ e $T_{C^{\prime \prime}}$. 
Seja $L$ um segmento transversal ao campo $\widetilde{X}(f)$ com extremos $a \in C$ e $b \in C^{\prime} . \tilde{L}$ é o segmento transversal a $\tilde{X}(g)$ que une os pontos $\tilde{a}=T_{C}(a)$ e $\tilde{b}=T_{C^{\prime}}$. Consideramos os pontos $o=L \cap \Gamma$ e $\tilde{o}=\tilde{L} \cap \widetilde{\Gamma}$.

Tratamos o caso em que $\Gamma$ e $\widetilde{\Gamma}$ são atratores.

Observar que uma trajetória $\gamma_{v}(t)$ de $\tilde{X}(f)$ que passa por $v \in C$ (respectivamente $v \in C^{\prime}$ ) intercepta o segmento $L$ em uma seqüência de pontos $v_{1}, \cdots, v_{n}, \cdots$ convergente a $\Gamma$. Da mesma maneira, uma trajetória $\gamma_{\bar{v}}(t)$ de $\widetilde{X}(g)$ que passa por $\tilde{v}=T_{C}(v)$, intercepta o segmento $\widetilde{L}$ na seqüência de pontos $\tilde{v}_{1}, \cdots, \tilde{v}_{n}, \cdots$ convergente a $\widetilde{\Gamma}$.

Então, definimos o homeomorfismo $\varphi: S \rightarrow S$ da seguinte maneira:

$$
\varphi(M)= \begin{cases}T_{C}(M) & \text { se } M \in C \\ Z_{\tilde{o} \tilde{o}}^{-1} \circ Z_{o o}(M) & \text { se } M \in \Gamma \\ Z_{\bar{v}_{i} \tilde{v}_{i+1}}^{-1} \circ Z_{v_{i} v_{i+1}}(M) & \text { se } M \in v_{i} v_{i+1} \\ T_{C^{\prime}}(M) & \text { se } M \in C^{\prime}\end{cases}
$$

onde oo e õõ são as trajetórias $\Gamma$ e $\widetilde{\Gamma}$. Um arco de trajetória $v_{i} v_{i+1}$ é levado ao arco $\tilde{v}_{i} \tilde{v}_{i+1}$ por razão de comprimento de arco.

Prova do Teorema 12. Seja $\widetilde{M^{*}}=\widetilde{M}-\bigcup_{k} C_{k}^{\circ}$ onde $C_{k}^{\circ}, k=1, \ldots, s$, é o interior de uma região crítica correspondente a um nó, foco ou ciclo limite.

Sejam $R_{i}$ e $\widetilde{R}_{i}, i=1, \ldots, r$ as regiões canônicas de $\widetilde{X}(f)$ e do campo $\widetilde{X}(g)$ suficientemente próximo de $\widetilde{X}(f)$ respectivamente. $\widetilde{R}_{i}$ é a região canônica correspondente a $R_{i}$. Consideramos $R_{i}^{*}=R_{i} \cap \widetilde{M}^{*}$ e $\widetilde{R}_{i}^{*}=\widetilde{R}_{i} \cap \widetilde{M}^{*}$.

Pelos Lemas 3.23 e 3.24, temos definidos os homeomorfismos $T_{C_{k}}: C_{k} \rightarrow$ $C_{k}$ e $T_{R_{i}^{*}}: R_{i}^{*} \rightarrow \widetilde{R}_{i}^{*}$. Estes homeomorfismos preservam o tipo de separatrizes de cada região canônica.

$\mathrm{Na}$ intersecção entre as regiões críticas e as regiões canônicas, e entre as regiões canônicas, os homeomorfismos são definidos de uma mesma maneira, isto é, por razão de comprimento de arco. 


\subsection{Exemplos de $X(f)$ estruturalmente estável}

Nesta seção apresentamos exemplos de campos $X(f) \in \Sigma^{n}$ que formam cada tipo de região canônica. Indicamos com $\alpha$ uma fonte, com $\omega$ um atrator representando um nó, um foco ou um ciclo limite, com $\gamma$ uma trajetória e com $p$ uma singularidade.

Tipo $i$. A fronteira da região $\partial R$ consiste de uma fonte e um atrator.

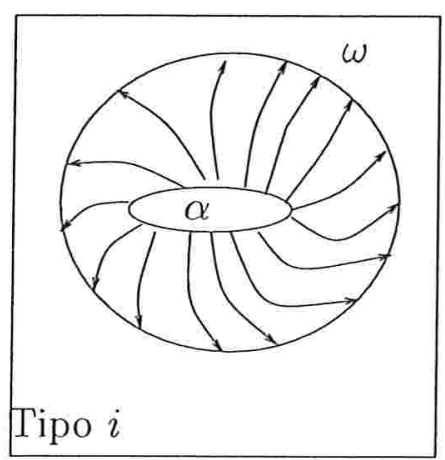

a)

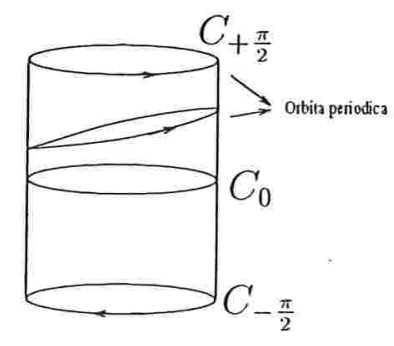

b)

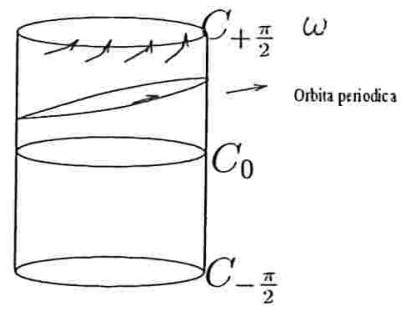

Figura 3.9: Região canônica Tipo i. a) e b) exemplos no cilindro.

Exemplo 3.1 Uma região limitada por dois ciclos limites circundando o cilindro sem conter nenhuma singularidade em seu interior. Ver figura 3.9 a). Esta situação aparece quando n é igual a 1 ou 2. Por exemplo, a equação estudada em [2] e [23]),

$$
x^{\prime \prime}+\alpha x^{\prime}+\beta+\sin (x)=0
$$

apresenta uma órbita periódica circundando o cilindro para certos valores dos parâmetros $\alpha$ e $\beta$.

Exemplo 3.2 Uma região limitada por um ciclo limite circundando o cilindro e um atrator no círculo $C_{ \pm \frac{\pi}{2}}$. Ver figura 3.9 b). Esta situação aparece quando $n$ é maior ou igual a 3 e o campo $X(f)$ não tem singularidades no infinito (i.e. $\tilde{X}(f)$ não tem singularidades ou pontos de tangências nos círculos $\left.C_{ \pm \frac{\pi}{2}}\right)$. 
Tipo ii. A fronteira da região $\partial R$ consiste de uma fonte, um atrator e somente uma trajetória.
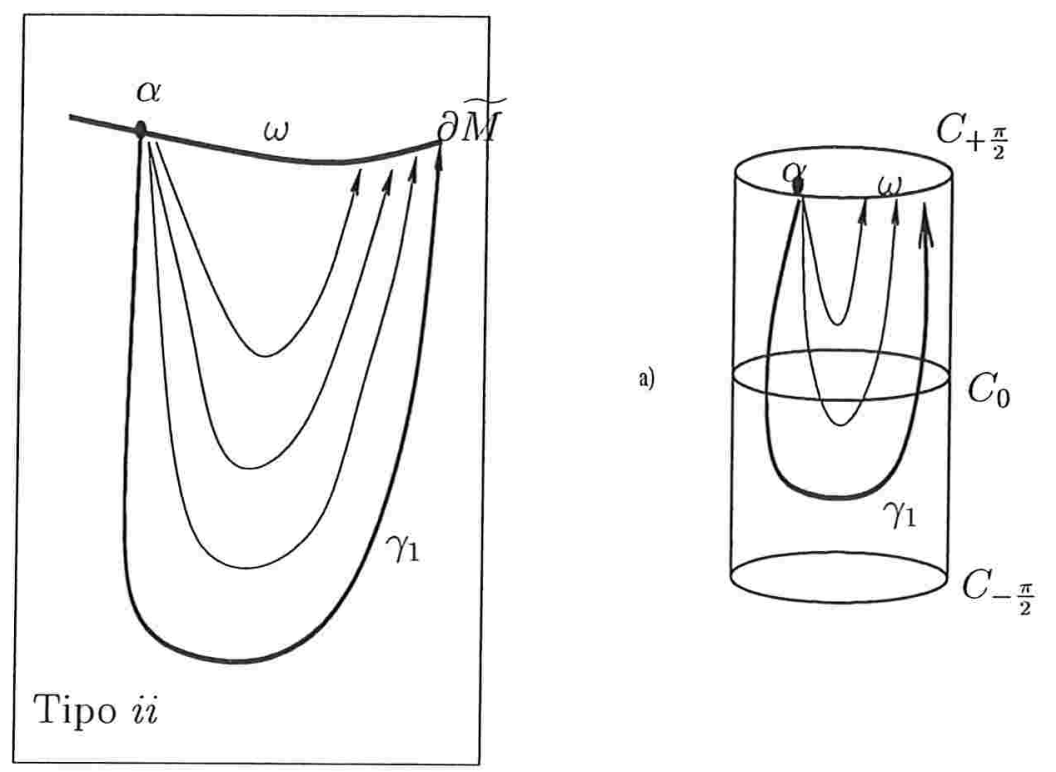

Figura 3.10: Região canônica Tipo ii. a) exemplo no cilindro.

Exemplo 3.3 Uma região limitada por um nó repulsor em $C_{+\frac{\pi}{2}}$ e a variedade fraca do nó cortando transversalmente a $\partial \widetilde{M}$. Ver figura 3.10 a). Esta situação aparece quando $n \geq 4$. 
Tipo iv. $\quad \partial R=\alpha \cup \omega \cup \gamma_{1} \cup \gamma_{2}$ onde $\gamma_{1}$ e $\gamma_{2}$ são separatrizes. Cada componente de fronteira pode ser reduzida a um ponto.
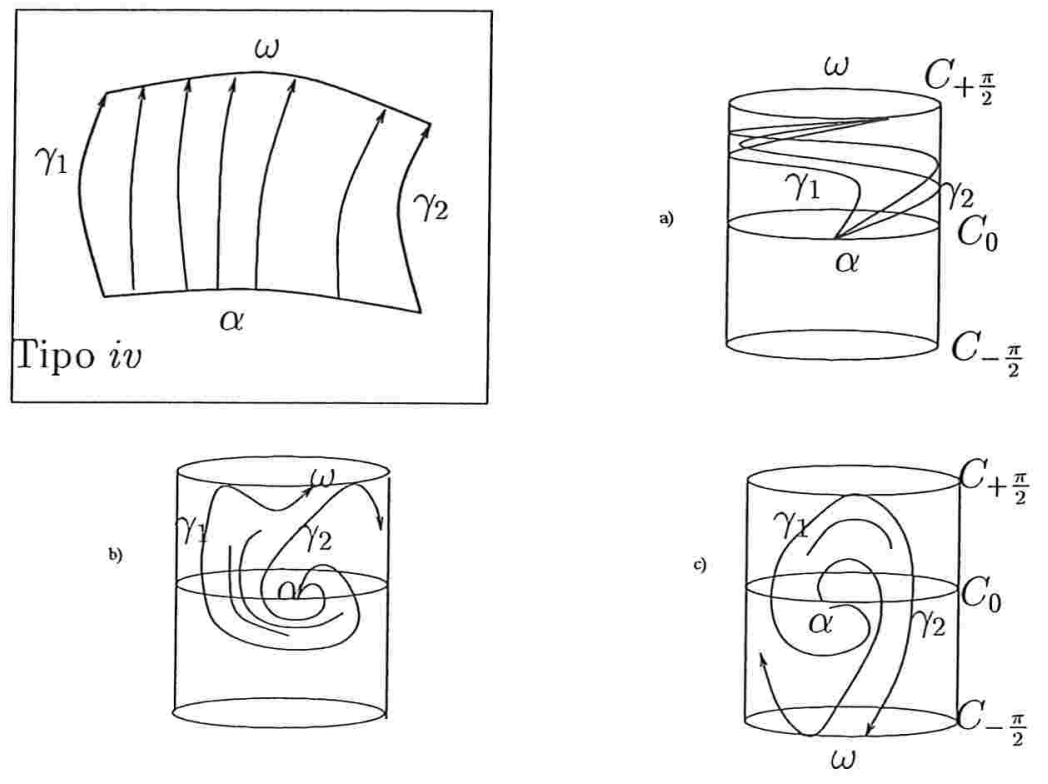

Figura 3.11: Região canônica Tipo iv. Três exemplos no cilindro.

Exemplo 3.4 Duas trajetórias que vêm de um nó em $C_{o}$ e chegam a uma singularidade do tipo nó em $C_{ \pm \frac{\pi}{2}}$. Ver figura 3.11 a). Esta situação aparece quando $n \geq 4$.

Exemplo 3.5 Duas trajetórias $\gamma_{1}$ e $\gamma_{2}$ tangentes a $C_{+\frac{\pi}{2}}$. Ver figura 3.11 b). Esta situação aparece quando $n=3$.

Exemplo 3.6 Duas trajetórias $\gamma_{1}$ e $\gamma_{2}$ tangentes a $C_{+\frac{\pi}{2}}$ e $C_{-\frac{\pi}{2}}$ respectivamente. Ver figura 3.11 c). Esta situação aparece quando $n=3$. 
Tipo $v$. A fronteira $\partial R$ consiste de uma singularidade $p_{1}$ e duas separatrizes $\gamma_{1}$ e $\gamma_{2}$ tendendo a ela, uma fonte $\alpha$ e um atrator $\omega$.

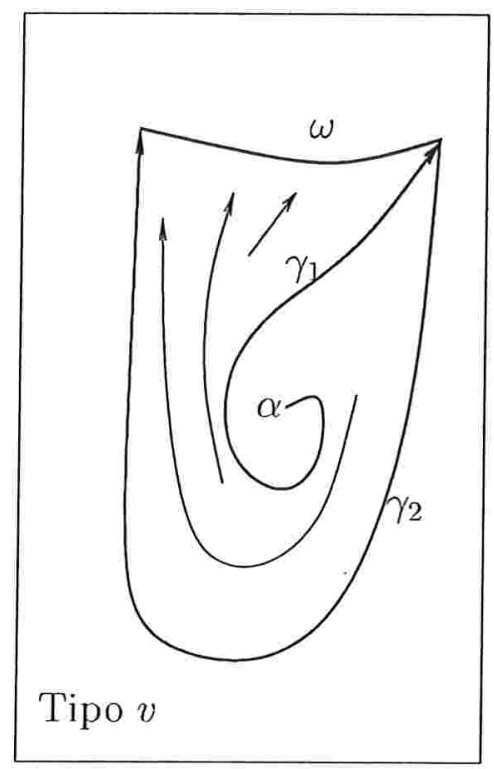

a)

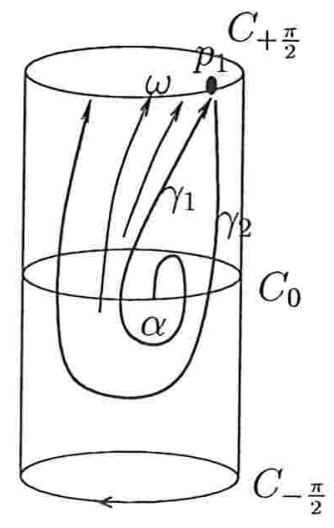

Figura 3.12: Região canônica Tipo v. a) exemplo no cilindro.

Exemplo 3.7 Uma sela ou sela-nó $p_{1}$ em $C_{+\frac{\pi}{2}}$, um atrator em $C_{ \pm \frac{\pi}{2}}$ e um repulsor em $C_{0}$. Ver figura 3.12. Esta situação aparece quando $n \geq 4$. 
Tipo vi. A fronteira $\partial R$ consiste de uma sela $p_{1}$ com duas separatrizes $\gamma_{1}$ e $\gamma_{2}$ tendendo a ela, uma fonte $\alpha$, um atrator $\omega$ e uma outra separatriz $\gamma_{3}$ que pode ser reduzida a um ponto.

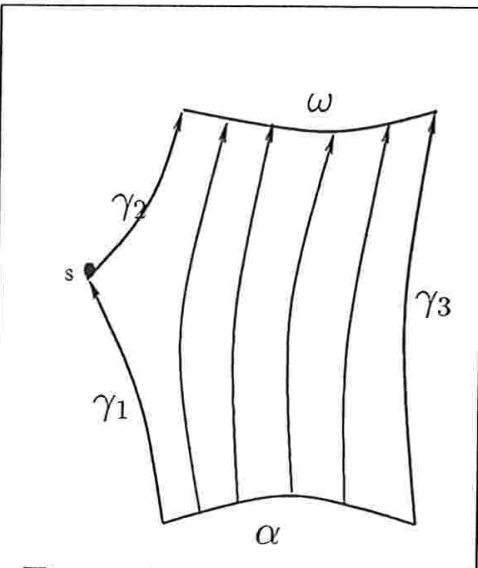

Tipo vi a)

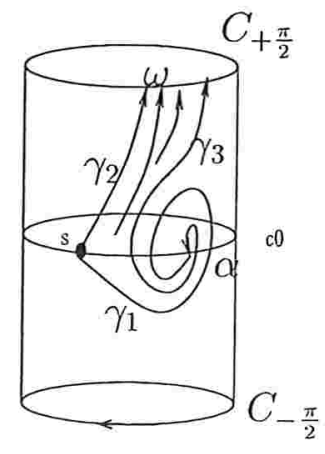

Figura 3.13: Região canônica T'ipo vi. Exemplos no cilindro.
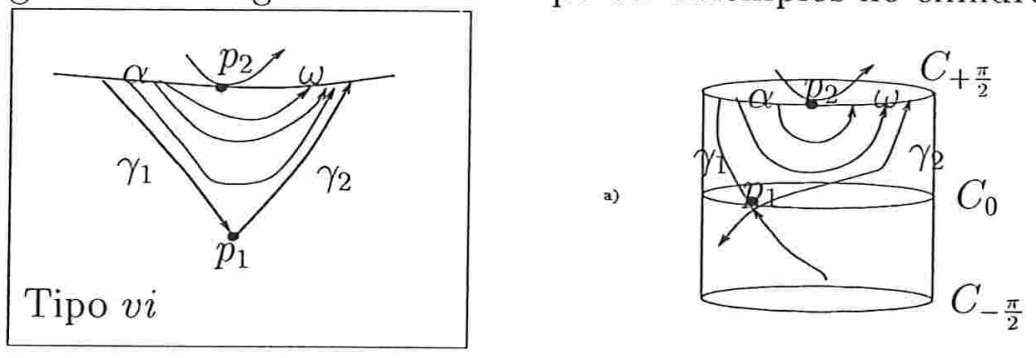

b)

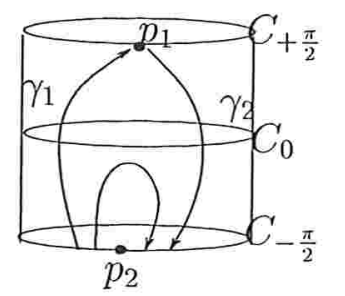

Figura 3.14: Região canônica tipo vi. Exemplos no cilindro onde $\gamma_{3}$ é reduzida a um ponto.

Exemplo 3.8 Uma sela $p_{1}$ e um foco (ou nó) em $C_{0}$ e um atrator em $C_{ \pm \frac{\pi}{2}}$. Ver figura 3.13. Esta situação aparece $\widetilde{X}(f)$ não tem singularidades ou pontos de tangências em $C_{ \pm \frac{\pi}{2}}$. Ver figura 3.13 a). 
Exemplo 3.9 Uma sela em $C_{0}$ com duas separatrizes tendendo $C_{+\frac{\pi}{2}}$ e um ponto de tangência externa em $C_{+\frac{\pi}{2}}$. Ver figura $3.14 a$ ). Esta situação acontece quando $n=3$.

Exemplo 3.10 Uma sela $(n=4)$ ou sela-nó $(n \geq 5) C_{+\frac{\pi}{2}}$ com duas separatrizes tendendo $C_{-\frac{\pi}{2}}$ e uma singularidade do tipo foco ou nó em $C_{+\frac{\pi}{2}}$. Ver figura $3.14 \mathrm{~b}$ ).

Tipo vii. $\partial R$ consiste de uma sela $p_{1}$ com três separatrizes $\gamma_{1}, \gamma_{2}$ e $\gamma_{3}$ tendendo à sela, uma fonte e um atrator.

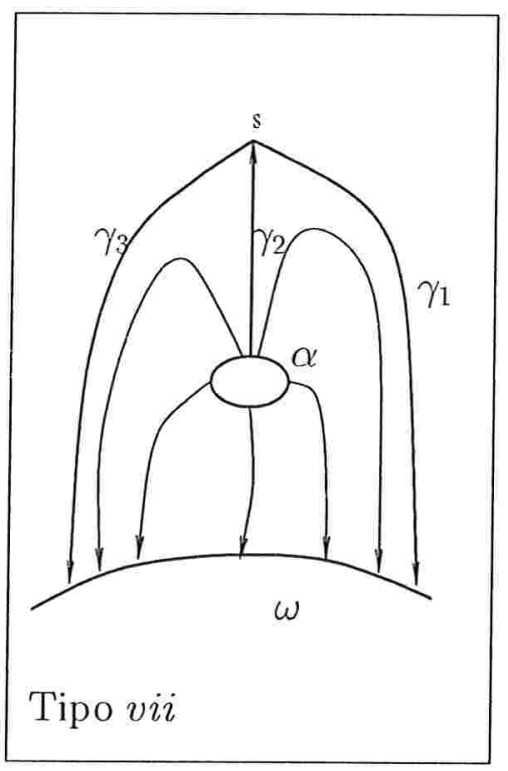

a)

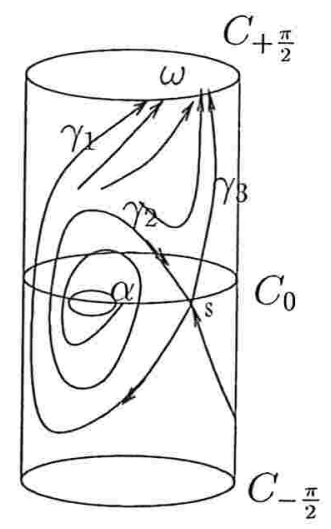

Figura 3.15: Região canônica Tipo vii. a) exemplo no cilindro.

Exemplo 3.11 Uma sela, duas separatrizes ilimitadas tendendo à sela e um ponto de tangência externa em $C_{ \pm \frac{\pi}{2}}$. Ver figura 3.15. 
Tipo viii. $\partial R=\left\{\alpha, \omega, p_{1}, \gamma_{1}, \gamma_{2}, p_{2}, \gamma_{3}\right\}$.
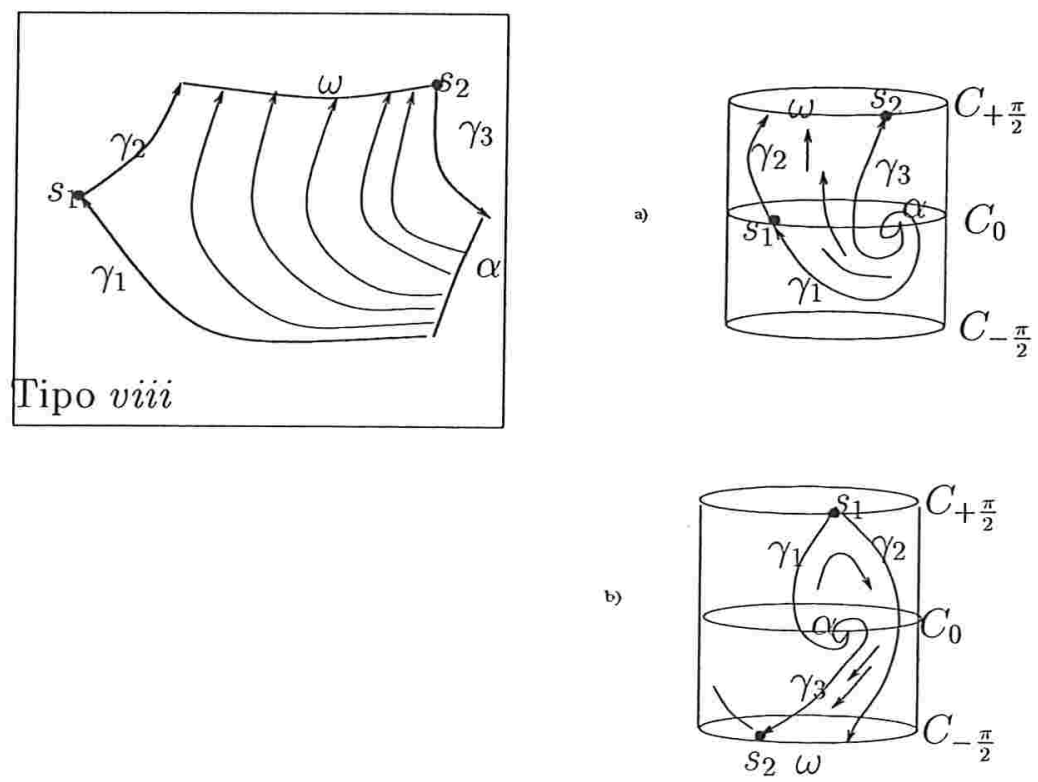

Figura 3.16: Região canônica Tipo viii. Exemplos no cilindro.

Exemplo 3.12 Uma sela em $C_{0}$ com duas separatrizes e uma sela ou sela-nó em $C_{ \pm \frac{\pi}{2}}$. Ver figura 3.16 a).

Exemplo 3.13 Uma sela $(n=4)$ ou sela-nó $(n>4)$ em $C_{+\frac{\pi}{2}}$ com duas separatrizes e uma sela $(n=4)$ ou sela-nó $(n>4)$ em $C_{-\frac{\pi}{2}}$. Ver figura 3.16 b). 
Tipo $i x . \partial R=\left\{\alpha, \omega, p_{1}, \gamma_{1}, \gamma_{2}, p_{2}, \gamma_{3}, \gamma_{4}\right\}$
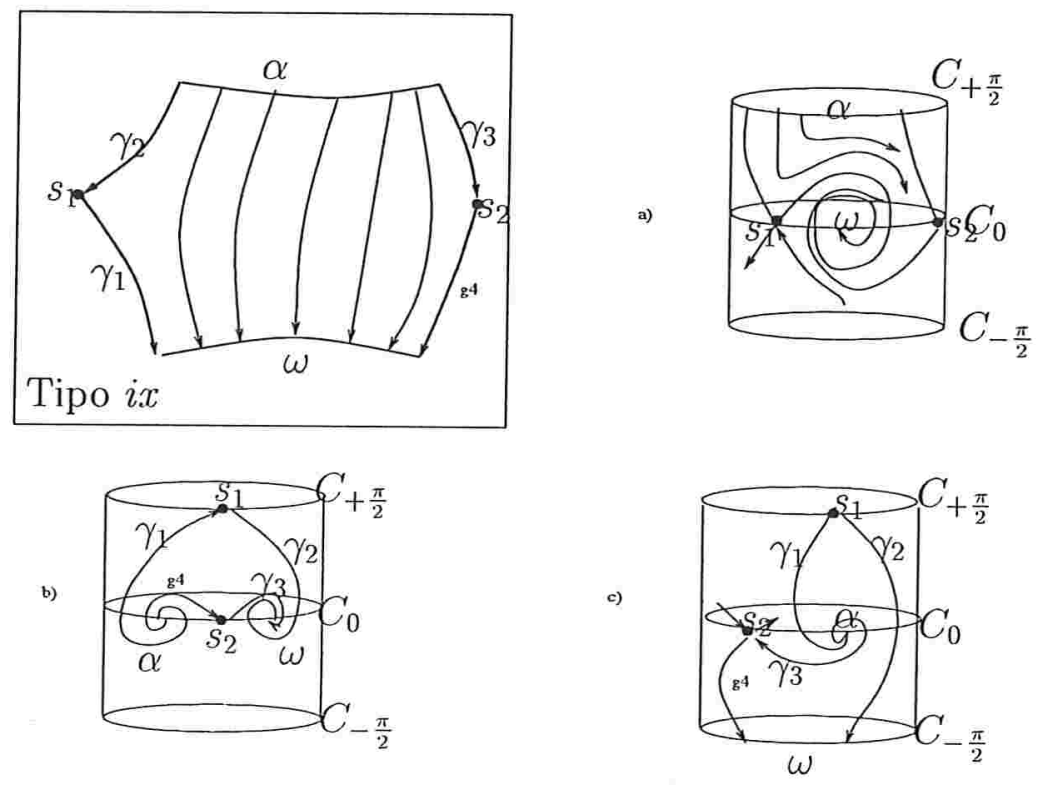

Figura 3.17: Região canônica Tipo $i x$. Exemplos no cilindro.

Exemplo 3.14 Duas sela em $C_{0}$ com uma separatriz cada tendendo a $C_{ \pm \frac{\pi}{2}}$. Ver figura 3.17 a).

Exemplo 3.15 Uma sela em $C_{0}$ com duas separatrizes tendendo a ela e uma sela $(n=4)$ ou sela-nó $(n \geq 5)$ em $C_{+\frac{\pi}{2}}$. Ver figura $\left.3.17 b\right)$.

Exemplo 3.16 Uma sela em $C_{0}$ com uma separatriz tendendo a $C_{ \pm \frac{\pi}{2}}$ e uma sela $(n=4)$ ou sela-nó $(n \geq 5)$ em $C_{ \pm \frac{\pi}{2}}$. Ver figura $\left.3.17 c\right)$. 


\section{Conclusões}

Neste trabalho caracterizamos os campos vetoriais $X(f)$ estruturalmente estáveis, definidos no cilindro $M=S^{1} \times \Re$, que provêm das equações diferenciais de segunda ordem da forma $x^{\prime \prime}=\sum_{i=0}^{n} a_{i}(x)\left(x^{\prime}\right)^{i}$ onde as funções coeficientes são periódicas de classe $C^{r}$.

Para cada $n$ fixo, consideramos o espaço $\mathcal{E}^{n, r}$ dos campos $X(f)$ munido da topologia dada pela norma $C^{r}$ das funções coeficientes. Encontramos um conjunto $\Sigma^{n}$ contido em $\mathcal{E}^{n, r}$ que caracteriza os campos estruturalmente estáveis para os seguintes casos:

- $n=1$ e $r \geq 2$

- $n=2,3$ e 4 , e $r \geq 1$

- $n \geq 5$ e $r \geq 2$

A genericidade de $\Sigma^{n}$ em $\mathcal{E}^{n, r}$ foi provada nas seções 3.1.2, 3.2.2, 3.3.2, 3.4 .2 e 3.5.2 e a caracterização dos campos estruturalmente estáveis foi provada nas seções 3.6.1 e 3.6.2.

Este trabalho constitui uma primeira etapa no entendimento do comportamento global das equações diferenciais de segunda ordem polinomiais com coeficientes periódicos. Demonstramos que aplicando uma compactificação do espaço de fase e do campo vetorial, podemos conhecer o comportamento qualitativo das equações perto do infinito, a partir de critérios expressos em termos das funções coeficientes.

Acreditamos que com base no presente trabalho possam ser abordadas questões mais complexas de interesse para as aplicações. Entre estas, mencionamos

- O estudo dos pontos de equilíbrio não-hiperbólicos, finitos e infinitos, e suas bifurcações.

- O estudo das equações diferenciais de segunda ordem seccionalmente diferenciáveis.

- A extensão das equações diferenciais de segunda ordem a mais variáveis de fase. 


\section{Referências Bibliográficas}

[1] Andronov, A. A., E. Leontovich, I. Gordon, and A. Maier: 1971, Theory of Bifurcations of Dynamic Systems on a Plane. Israel Program for Scientific Translations.

[2] Andronov, A. A., A. A. Vitt, and S.E.Khaikin: 1966, Theory of Oscillators. Pergamon press.

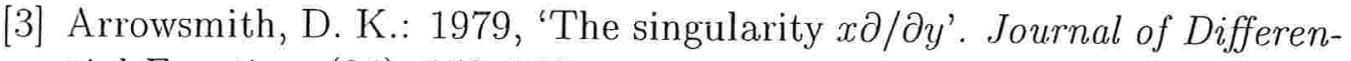
tial Equations (34), 153-166.

[4] Barreto, A. C.: 1964, 'Estabilidade Estrutural das Equações Diferenciais da Forma $x^{\prime \prime}=f\left(x, x^{\prime}\right)$.' Tese de Doutorado, IMPA.

[5] Brunella, M. and M. Miari: 1990, 'Topological Equivalence of a Plane Vector Field with its Principal Part Defined through Newton Polyhedra'. Journal of Differential Equations (85), 338-366.

[6] Camacho, C., M. Krych, R. Mañe, and Z. Nictecki: 1983, 'An extension of Peixoto's structural stability theorem to open surface with finite genus'. Lecture Notes in Math. (1007).

[7] Chicone, C.: 1999, Ordinary differential equations with applications, No. 34 in Text Applied Math. Springer Verlag.

[8] Dumortier, F.: 1977, 'Singularities of vector fields on the plane'. Journal of Differential Equations 23, 53-106.

[9] Dumortier, F.: 1995, 'Local study of planar vector fields: Singularities and their unfoldings'. In: E. von Groesen and E. de Jager (eds.): Studies in Mathematical Physics 2, Structures in dynamic, finite dimensional, deterministic studies.

[10] Fernandez, P. J.: 1976, Medida e Integração, Projeto Euclides. IMPA. Segunda Edição. 
[11] Golubitsky, M. and V. Guillemin: 1973, Stable Mappings and Their Singularities, Vol. 14 of Graduate Texts in Mathematics. Springer-Verlag.

[12] González-Velasco, E. A.: 1969, 'Generic Properties of Polynomial Vector Fields at Infinity'. Transactions A.M.S. 143, 201-222.

[13] Guckenheimer, J. and P. Holmes: 1983, Nonlinear Oscillations, Dynamical Systems, and Bifurcations of Vector Fields, Vol. 42 of Applied Mathematical Sciences. Springer Verlag, corrected fifth printing, 1997 edition.

[14] Kotus, J.: 1990, 'Global $C^{r}$ structural stability of vector fields on open surface with finite genus'. Proceedings of the American Mathematical Society 108(4).

[15] Kotus, J., M. Krych, and Z. Nitecki: 1982, 'Global structural stability of flows on open surfaces'. Memoirs of the American Mathematical Society $37(261)$.

[16] Neumann, D. A.: 1975, 'Classification of continuous flows on 2manifolds'. Proceedings of the American Mathematical Society 48(1), $73-81$.

[17] Palis, J. and W. de Melo: 1978, Introdução aos sistemas dinâmicos. Projeto Euclides. IMPA.

[18] Peixoto, M. C. and M. M. Peixoto: 1959, 'Structural Stability in the Plane with Enlarged Boundary Conditions'. Anais da Academia Brasileira de Ciencias 31(2), 135-160.

[19] Sotomayor, J. M.: 1981, Curvas Definidas por Equações Diferenciais no Plano. 13 Colóquio Brasileiro de Matemática.

[20] Sotomayor, J. M.: 1982, 'Structurally Stable Second Order Differential Equations'. Lecture Notes in Math. (957), 284--301.

[21] Sotomayor, J. M.: 1985, 'Stable planar vector fields'. Rev. Mat. Iberoamericana 1.

[22] Teixeira, M. A.: 1977, 'Generic Bifurcation on Manifolds with Boundary'. Journal Differential Equations 25(1).

[23] Tricomi, F.: 1933, 'Integrazione di un'equazione differenziale presentatasi in elettrotecnica'. Annali della Scuola Norm. Sup. Pisa 2(2), 1-20. 